\title{
Development of high-spatial and high-mass resolution mass spectrometric imaging (MSI) and its application to the study of small metabolites and endogenous molecules of plants
}

\author{
by
}

\section{Ji Hyun Jun}

A dissertation submitted to the graduate faculty

in partial fulfillment of the requirements for the degree of

DOCTOR OF PHILOSOPHY

\author{
Major: Physical Chemistry \\ Program of Study Committee: \\ Young Jin Lee, Major Professor \\ Ning Fang \\ Mark S. Gordon \\ Robert S. Houk \\ Theresa Windus
}

Iowa State University

Ames, Iowa

2012

Copyright $@$ Ji Hyun Jun, 2012. All rights reserved. 
To My Families and Friends 
TABLE OF CONTENTS

\begin{tabular}{lr} 
ABSTRACT & $\mathbf{v}$ \\
CHAPTER 1. GENERAL INTRODUCTION & $\mathbf{1}$ \\
\hline Dissertation Organization & 1 \\
General Introduction & 1 \\
References & 7
\end{tabular}

CHAPTER 2. HIGH-SPATIAL AND HIGH-MASS RESOLUTION IMAGING OF SURFACE METABOLITES OF ARABIDOPSIS THALIANA BY LASER DESORPTION-IONIZATION MASS SPECTROMETRY USING COLLOIDAL SILVER

Abstract

Introduction

Experimental Section

Results and Discussion

Conclusions

Acknowledgement

References

Figure Captions

CHAPTER 3. MASS SPECTROMETRIC IMAGING ON VARIOUS ORGANS OF ARABIDOPSIS MUTANT WITH HIGH SPATIAL AND HIGH MASS RESOLUTION FOR REVELATION OF PLANT METABOLITES DISTRIBUTION ALTERED BY GENETIC MUTATION

Abstract

Introduction

Experimental Section

Results and Discussion

Conclusions

Acknowledgement 73

References 76

Figure Captions 
CHAPTER 4. ENZYMATIC REMOVAL OF CELL WALLS OF THE INTERNAL CELLS EXPOSED THROUGH VIBRATOME SECTIONING AND MASS SPECTROMETRIC IMAGING OF INTERNAL CELL METABOLITES

Abstract

Introduction

Experimental Section

Results and Discussion

Conclusions

Acknowledgement

102

References

Figure Captions

CHAPTER 5. DEVELOPING A MALDI MS IMAGING ASSAY TO LOCALIZE CHLOROPLASTS AND QUANTIFY THE ABUNDANCES OF CHLOROPHYLL $a$ AT THE SINGLE ORGANELLE LEVEL

Abstract

Introduction

Experimental Section

Results and Discussion

Conclusions

Acknowledgement

References

Figure Captions

CHAPTER 6. GENERAL CONCLUSIONS

APPENDIX 1. SUPPORTING FIGURES FOR CHAPTER 2

Figure Captions

APPENDIX 2. SUPPORTING FIGURES FOR CHAPTER 3

Figure Captions

APPENDIX 3. SUPPORTING FIGURES FOR CHAPTER 5 


\begin{abstract}
High-spatial and high-mass resolution laser desorption ionization (LDI) mass spectrometric (MS) imaging technology was developed for the attainment of MS images of higher quality containing more information on the relevant cellular and molecular biology in unprecedented depth. The distribution of plant metabolites is asymmetric throughout the cells and tissues, and therefore the increase in the spatial resolution was pursued to reveal the localization of plant metabolites at the cellular level by MS imaging. For achieving high-spatial resolution, the laser beam size was reduced by utilizing an optical fiber with small core diameter $(25 \mu \mathrm{m})$ in a vacuum matrix-assisted laser desorption ionization-linear ion trap (vMALDI-LTQ) mass spectrometer. Matrix application was greatly improved using oscillating capillary nebulizer. As a result, single cell level spatial resolution of $\sim 12 \mu \mathrm{m}$ was achieved. MS imaging at this high spatial resolution was directly applied to a whole Arabidopsis flower and the substructures of an anther and single pollen grains at the stigma and anther were successfully visualized. MS imaging of high spatial resolution was also demonstrated to the secondary roots of Arabidopsis thaliana and a high degree of localization of detected metabolites was successfully unveiled. This was the first MS imaging on the root for molecular species. MS imaging with high mass resolution was also achieved by utilizing the LTQ-Orbitrap mass spectrometer for the direct identification of the surface metabolites on the Arabidopsis stem and root and differentiation of isobaric ions having the same nominal mass with no need of tandem mass spectrometry (MS/MS).
\end{abstract}


MS imaging at high-spatial and high-mass resolution was also applied to cer1 mutant of the model system Arabidopsis thaliana to demonstrate its usefulness in biological studies and reveal associated metabolite changes in terms of spatial distribution and/or abundances compared to those of wild-type. The spatial distribution of targeted metabolites, mainly waxes and flavonoids, was systematically explored on various organs, including flowers, leaves, stems, and roots at high spatial resolution of $\sim 12-50 \mu \mathrm{m}$ and the changes in the abundance level of these metabolites were monitored on the cer 1 mutant with respect to the wild-type. This study revealed the metabolic biology of $C E R 1$ gene on each individual organ level with very detailed high spatial resolution. The separate MS images of isobaric metabolites, i.e. C29 alkane vs. C28 aldehyde could be constructed on both genotypes from MS imaging at high mass resolution. This allows tracking of abundance changes for those compounds along with the genetic mutation, which is not achievable with low mass resolution mass spectrometry. This study supported previous hypothesis of molecular function of $C E R 1$ gene as aldehyde decarbonylase, especially by displaying hyper accumulation of aldehydes and C30 fatty acid and decrease in abundance of alkanes and ketones in several plant organs of cer 1 mutant.

The scope of analytes was further directed toward internal cell metabolites from the surface metabolites of the plant. MS profiling and imaging of internal cell metabolites were performed on the vibratome section of Arabidopsis leaf. Vibratome sectioning of the leaf was first conducted to remove the surface cuticle layer and it was followed by enzymatic treatment of the section to induce the digestion of primary cell walls, middle lamella, and expose the internal cells underneath to the surface for detection with the laser by LDI-MS. The subsequent MS imaging onto the enzymatically treated vibratome section allowed us to map the distribution of the metabolites in the internal cell layers, linolenic acid (C18:3 FA) and linoleic acid (C18:2 FA). 
The development of an assay for relative quantification of analytes at the single subcellular/organelle level by LDI-MS imaging was attempted and both plausibility and significant obstacles were seen. As a test system, native plant organelle, chloroplasts isolated from the spinach leaves were used and the localization of isolated chloroplasts dispersed on the target plate in low density was monitored by detecting the ion signal of chlorophyll $a$ (Chl $a$ ) degradation products such as pheophytin $a$ and pheophobide $a$ by LDI-MS imaging in combination with fluorescence microscopy. The number of chloroplasts and their localization visualized in the MS image exactly matched those in the fluorescence image especially at low density, which first shows the plausibility of single-organelle level quantification of analytes by LDI-MS. The accumulation level of $\mathrm{Chl} a$ within a single chloroplast detected by LDI-MS was compared to the fluorescence signal on a pixel-to-pixel basis to further confirm the correlations of the accumulation levels measured by two methods. The proportional correlation was observed only for the chloroplasts which do not show the significant leakage of chlorophyll indicated by MS ion signal of Chl $a$ degradation products and fluorescence signal, which was presumably caused by the prior fluorescence measurement before MS imaging. Further investigation is necessary to make this method more complete and develop LDI-MS imaging as an effective analytical tool to evaluate a relative accumulation of analytes of interest at the single subcellular/organelle level. 


\section{CHAPTER 1. GENERAL INTRODUCTION}

\section{Dissertation Organization}

This dissertation begins with general introduction of laser desorption ionization (LDI) mass spectrometry (MS) and mass spectrometric imaging (MSI) for profiling and imaging of small molecules. The following four chapters are presented as separated chapters from either a published paper or manuscripts in preparation. Chapter two and three are based on the development of the high-spatial and high-mass resolution LDI-MS imaging technique and its application to study mainly the surface metabolites of Arabidopsis thaliana and underlying biology. Chapter four presents LDI-MS imaging of internal cell metabolites. Chapter five is related to the development of LDI-MS imaging as an assay for relative quantification of analytes at the single subcellular/organelle level. Each chapter is followed by tables, cited literature, and figures. Chapter six contains the general conclusions. The three appendixes are presented at the end after all chapters and include the supporting figures for chapter one, two, and four. The dissertation is finished with acknowledgements.

\section{General Introduction}

Mass spectrometry (MS) is a useful analytical technique and has been applied to many scientific studies from its invention in the first decade of the $20^{\text {th }}$ century ${ }^{1}$. There are many ionization sources utilized in MS such as electron ionization (EI) ${ }^{2}$, chemical ionization (CI) ${ }^{3}$, atmospheric pressure chemical ionization (APCI) ${ }^{4}$, electrospray (ESI) ${ }^{5}$, atmospheric pressure photo ionization (APPI) ${ }^{6}$, fast atom bombardment (FAB) ${ }^{7}$ and so on. 
Among these, matrix-assisted laser desorption/ionization (MALDI) has been recognized as an effective soft ionization method to detect and analyze a variety of biomolecules directly from the biological materials of interest ${ }^{1}$ since developed in the 1980 s by Hillenkamp and Karas et al. ${ }^{8,9}$. The matrix has a strong absorption at the laser wavelength used and plays an important role in absorbing the laser energy. The analytes molecules are isolated through the explosive desorption of matrix crystals embedding the analytes. This induces a soft desorption of the matrix and analytes without a significant degradation of analytes because analytes are incorporated in the matrix crystals. The ionization of analytes is enhanced by chemical reactions in the laser plume ${ }^{1 \text {, }}$ ${ }^{10-14}$. Because MALDI minimizes the fragmentation of intact analyte molecule ${ }^{1}$ and mainly produces singly-charged ions, spectral complexity is minimized, which makes it easy to interpret the mass spectral data ${ }^{15}$. Due to many additional attractive features of MALDI-MS including a high salt or contaminants tolerance, high sensitivity, relatively low requirements of sample, and capability of direct analysis of mixtures ${ }^{11,16}$, its variety of uses range from small molecules such as drugs or metabolites to larger biomolecules including peptides, proteins, and lipids ${ }^{1,17-20}$.

In MALDI-MS analysis, matrix choice and sample preparation are important because these factors critically affect the ionization efficiency, fragmentation, and quantification ${ }^{16}$. It is noteworthy to mention that a significant problem frequently occurs especially for the analysis of small molecules $(<1,000 \mathrm{Da})$ because the matrix produces interfering peaks in the low mass region and suppresses the ion signal of small molecules. There have been many attempts to overcome this problem including adoption of high molecular weight matrixes ${ }^{21,22}$, inorganic matrixes ${ }^{23,24}$, matrix-free approaches such as desorption/ionization on silicon (DIOS) MS ${ }^{25}$ and nanostructure initiator MS (NIMS) ${ }^{26}$, addition of surfactant to matrix ${ }^{27,28}$ and use of binary matrixes ${ }^{29}$. Typical organic acid matrixes such as 2, 5-dihydroxybenzoic acid (DHB) applied by 
the most common means called dried-droplet method ${ }^{17}$ forms "sweet spot" which leads to significant inhomogeneity of sample and matrix, and hence poor shot-to-shot and spot-to-spot reproducibility ${ }^{16}$. To reduce these problems arising from the heterogeneous crystallization of analyte and matrix and to increase the quality of MALDI experiments, proper sample preparation is necessary. There are many approaches for sample preparation to improve the sample homogeneity such as fast evaporation method ${ }^{30}$, crystal crushing method ${ }^{31,32}$, seed-layer method ${ }^{33}$, application of co-matrixes (i.e., fucose, fructose, 5-methoxysalicylic acid) ${ }^{34-37}$, microcrystalline depositions through sophisticated spotting devices such as electrospray ${ }^{38-41}$ or piezoelectric micro-dispensers ${ }^{42-44}$, and sublimation ${ }^{45-47}$. Additionally, solvent composition, $\mathrm{pH}$ or temperature of the solution, and molar matrix-to-analyte (M/A) ratio also influence the cocrystallization of analyte and matrix ${ }^{16}$. Therefore, a careful design of sample preparation needs to be taken into account for each analysis considering the type of samples to be analyzed, analytes of interest, and matrixes. The ultimate benefit is the decrease in the time and the cost consumed for each analysis, better reproducibility, increased sensitivity, higher resolution, and increased tolerance to contamination ${ }^{33}$.

Mass spectrometric (MS) imaging emerged from a demand for the information about the spatial distribution of the biomolecules detected by mass spectrometry ${ }^{1}$ (Figure 1). Since its introduction in 1997 by Caprioli and co-workers ${ }^{48}$, MALDI-MS imaging has undergone many technical improvements of devices for sample preparation, instrumentation (i.e., laser spot size, sampling rate), and software for data acquisition and analysis ${ }^{1,49}$.

For the data acquisition in MALDI-MS imaging, the biological sample coated with matrix is rastered at the fixed step size by moving the sample plate placed in the vacuum of the mass 
spectrometer while the laser is fixed at the optimized position to the inlet of mass spectrometer ${ }^{1}$. The MS images can be constructed through the MALDI imaging software by analyzing the intensity profile of a particular peak selected from the mass spectra across the sample surface subjected to laser rastering. MALDI-MS imaging is a label-free detection method which enables the simultaneous detection of various types of molecules directly from the biological surface without the need of prior knowledge on the sample ${ }^{49}$. The benefits of having MALDI as an ionization source in MS have been already mentioned above, and those advantages are also applied to MS imaging with MALDI. The application of MALDI-MS imaging varies enormously due to many attractive features. Therefore it has been used for the study of pharmaceuticals, lipids, metabolites, peptides, and proteins ${ }^{50}$.

Besides MALDI-MS imaging, there are other types of ionization techniques used for MS imaging such as secondary ion mass spectrometry (SIMS)-based imaging, desorption electrospray ionization (DESI)-MS imaging, and less frequently laser ablation electrospray ionization (LAESI)-MS imaging. SIMS-based imaging in which focused primary ion beam (i.e., $\left.\mathrm{C}_{60}{ }^{+}, \mathrm{Ar}^{+}, \mathrm{Xe}^{+}, \mathrm{In}^{+}\right)$is used to release secondary ions from the sample surface enables high spatial resolution down to a submicron level. The practical mass range of SIMS is limited to $\mathrm{m} / \mathrm{z} 1000$ due to the extensive fragmentation of surface molecules, which is because the high energy is deposited onto the surface from the focused primary ion. Therefore, SIMS is typically suitable for the measurement of elements or small molecules ${ }^{50}$. DESI-MS imaging was developed in 2004 by Cooks and co-workers ${ }^{51}$ and uses charged electrosprayed solvent droplets to generate the desorbed molecules from the sample surface. It is operated in the ambient condition and no additional sample preparation is necessary ${ }^{50}$. However, its drawback as used for MS imaging is definitely low spatial resolution. Laser ablation electrospray ionization 
(LAESI)-MS imaging is also conducted under atmospheric pressure. It is mainly targeted for the biological sample with water content and utilizes a focused mid-IR laser beam $(2940 \mathrm{~nm}$ wavelength) to excite the $\mathrm{OH}$ vibrations in water molecules followed by the release of intact sample particulates into the electrospray for postionization ${ }^{52,53}$. This ionization method also simplifies the sample preparation but its lateral resolution is presently only about $300-350 \mu \mathrm{m}^{53}$, 54.

In MALDI-MS imaging, (1) laser spot size and (2) matrix homogeneity are most important factors significantly affecting the spatial resolution of the MS images. Many attempts have been made to reduce the laser spot size including use of a pinhole ${ }^{48}$, multiple focusing lenses ${ }^{55}$, coaxial laser illumination ${ }^{56}$, or utilization of an optical fiber ${ }^{57,58}$. Previously, colloidal silver and graphite have been adopted in our group as matrixes in LDI-MS imaging for profiling and imaging of plant metabolites ${ }^{57,59}$. Unlike the organic acid matrixes conventionally used in MALDI-MS, these matrixes do not form "sweet spot" from which the good signals are obtained for analytes. "Sweet spot" leads to the significant inhomogeneity of the matrix, and hence with these novel matrixes, MS imaging could be performed overcoming the limits in the spatial resolution restrained by matrix inhomogeneity with conventional matrixes. The following chapters include more detailed description of the efforts to improve the spatial resolution in MS imaging in terms of these two factors. The importance of high mass resolution, another issue besides spatial resolution, in MS imaging is also described in detail in the following chapters with respect to direct identification of analytes detected by MS through chemical composition analysis and differentiation of isobaric ions having the same nominal $\mathrm{m} / \mathrm{z}$ values. 


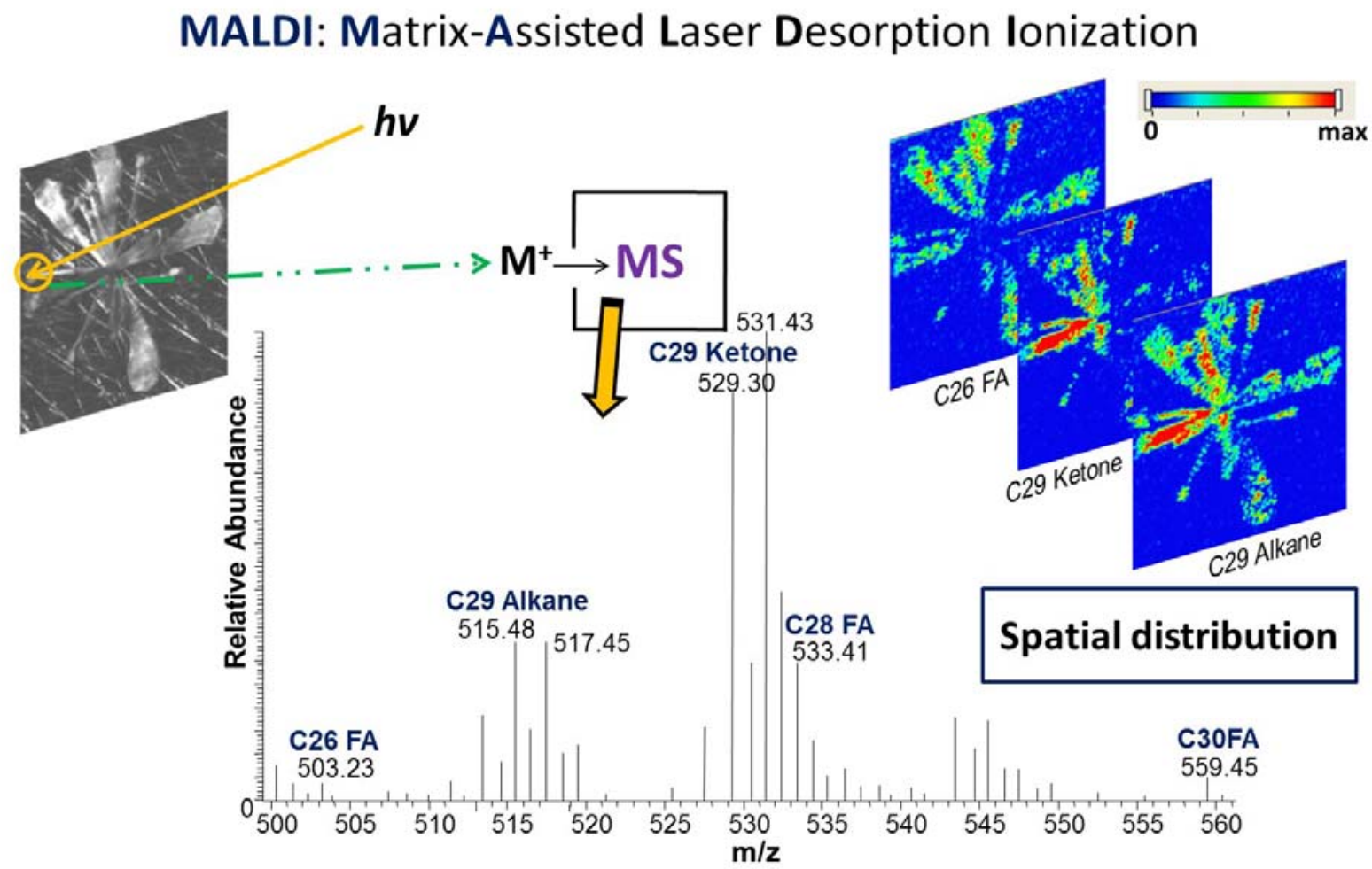

Figure 1. Principle of (MALDI) MS imaging and the information obtainable by MS imaging: qualitative, semi-quantitative information on the analytes, and spatial distribution of the analytes. 


\section{References}

1. $\quad$ Chughtai, K.; Heeren, R., Chemical Reviews 2010, 110 (5), 3237-3277.

2. $\quad$ Fales, H. M.; Milne, G. W.; Pisano, J. J.; Brewer, H. B., Jr.; Blum, M. S.; MacConnell, J. G.; Brand, J.; Law, N., Recent progress in hormone research 1972, 28, 591-626.

3. Munson, M. S. B.; Field, F. H., Journal of the American Chemical Society 1966, 88 (12), 2621-\&.

4. $\quad$ Asselin, M. J. F.; Pare, J. J. R., Organic Mass Spectrometry 1981, 16 (6), 275-278.

5. $\quad$ Fenn, J. B.; Mann, M.; Meng, C. K.; Wong, S. F.; Whitehouse, C. M., Science 1989, 246 (4926), 64-71.

6. Takino, M.; Tanaka, T.; Yamaguchi, K.; Nakahara, T., Food Additives and Contaminants Part a-Chemistry Analysis Control Exposure \& Risk Assessment 2004, 21 (1), 76-84.

7. Williams, D. H.; Bradley, C.; Bojesen, G.; Santikarn, S.; Taylor, L. C. E., Journal of the American Chemical Society 1981, 103 (19), 5700-5704.

8. $\quad$ Karas, M.; Bachmann, D.; Hillenkamp, F., Analytical Chemistry 1985, 57 (14), 29352939.

9. $\quad$ Karas, M.; Bachmann, D.; Bahr, U.; Hillenkamp, F., International Journal of Mass Spectrometry and Ion Processes 1987, 78, 53-68.

10. Karas, M.; Bahr, U.; Hillenkamp, F., International Journal of Mass Spectrometry and Ion Processes 1989, 92, 231-242.

11. Karas, M.; Kruger, R., Chemical Reviews 2003, 103 (2), 427-439.

12. $\quad$ Ehring, H.; Karas, M.; Hillenkamp, F., Organic Mass Spectrometry 1992, 27 (4), 472480 .

13. Liao, P.; Allison, J., Journal of Mass Spectrometry 1995, 30 (3), 408-423.

14. Zenobi, R.; Knochenmuss, R., Mass Spectrometry Reviews 1998, 17 (5), 337-366.

15. El-Aneed, A.; Cohen, A.; Banoub, J., Applied Spectroscopy Reviews 2009, 44 (3), 210230.

16. Tholey, A.; Heinzle, E., Analytical and Bioanalytical Chemistry 2006, 386 (1), 24-37.

17. Karas, M.; Hillenkamp, F., Analytical Chemistry 1988, 60 (20), 2299-2301.

18. Aebersold, R.; Goodlett, D., Chemical Reviews 2001, 101 (2), 269-295.

19. Lahm, H.; Langen, H., Electrophoresis 2000, 21 (11), 2105-2114. 
20. Gevaert, K.; Vandekerckhove, J., Electrophoresis 2000, 21 (6), 1145-1154.

21. Huang, J.; Yuan, C.; Shiea, J.; Chen, Y., Journal of Analytical Toxicology 1999, 23 (5), 337-342.

22. Ayorinde, F.; Hambright, P.; Porter, T.; Keith, Q., Rapid Communications in Mass Spectrometry 1999, 13 (24), 2474-2479.

23. Sluszny, C.; Yeung, E. S.; Nikolau, B. J., J. Am. Soc. Mass Spectrom. 2005, 16 (1), $107-$ 15.

24. McLean, J.; Stumpo, K.; Russell, D., Journal of the American Chemical Society 2005, 127 (15), 5304-5305.

25. Wei, J.; Buriak, J.; Siuzdak, G., Nature 1999, 399 (6733), 243-246.

26. Northen, T.; Yanes, O.; Northen, M.; Marrinucci, D.; Uritboonthai, W.; Apon, J.;

Golledge, S.; Nordstrom, A.; Siuzdak, G., Nature 2007, 449 (7165), 1033-U3.

27. Grant, D.; Helleur, R., Rapid Communications in Mass Spectrometry 2008, 22 (2), 156164.

28. $\quad$ Grant, D.; Helleur, R., Rapid Communications in Mass Spectrometry 2007, 21 (6), 837845.

29. Zhong, G.; Lin, H., Analytical and Bioanalytical Chemistry 2007, 387 (5), 1939-1944.

30. Nicola, A. J.; Gusev, A. I.; Proctor, A.; Jackson, E. K.; Hercules, D. M., Rapid Commun Mass Spectrom 1995, 9 (12), 1164-71.

31. Westman, A.; Demirev, P.; Huthfehre, T.; Bielawski, J.; Sundqvist, B. U. R., International Journal of Mass Spectrometry and Ion Processes 1994, 130 (1-2), 107-115.

32. Xiang, F.; Beavis, R. C., Rapid Communications in Mass Spectrometry 1994, 8 (2), 199204.

33. Onnerfjord, P.; Ekström, S.; Bergquist, J.; Nilsson, J.; Laurell, T.; Marko-Varga, G., Rapid Commun Mass Spectrom 1999, 13 (5), 315-22.

34. Gusev, A. I.; Wilkinson, W. R.; Proctor, A.; Hercules, D. M., Anal Bioanal Chem 1996, 354 (4), 455-63.

35. Gusev, A.; Wilkinson, W.; Proctor, A.; Hercules, D., Analytical Chemistry 1995, 67 (6), 1034-1041.

36. Laugesen, S.; Roepstorff, P., J Am Soc Mass Spectrom 2003, 14 (9), 992-1002.

37. Distler, A. M.; Allison, J., Anal Chem 2001, 73 (20), 5000-3. 
38. Hensel, R. R.; King, R. C.; Owens, K. G., Rapid Communications in Mass Spectrometry 1997, 11 (16), 1785-1793.

39. Hanton, S. D.; Hyder, I. Z.; Stets, J. R.; Owens, K. G.; Blair, W. R.; Guttman, C. M.; Giuseppetti, A. A., Journal of the American Society for Mass Spectrometry 2004, 15 (2), 168179.

40. Wei, H.; Nolkrantz, K.; Powell, D. H.; Woods, J. H.; Ko, M. C.; Kennedy, R. T., Rapid Communications in Mass Spectrometry 2004, 18 (11), 1193-1200.

41. Wagner, M.; Varesio, E.; Hopfgartner, G., Journal of Chromatography B-Analytical Technologies in the Biomedical and Life Sciences 2008, 872 (1-2), 68-76.

42. Little, D. P.; Cornish, T. J.; Odonnell, M. J.; Braun, A.; Cotter, R. J.; Koster, H., Analytical Chemistry 1997, 69 (22), 4540-4546.

43. Allmaier, G., Rapid Communications in Mass Spectrometry 1997, 11 (14), 1567-1569.

44. Onnerfjord, P.; Nilsson, J.; Wallman, L.; Laurell, T.; Marko-Varga, G., Analytical Chemistry 1998, 70 (22), 4755-4760.

45. Hankin, J. A.; Barkley, R. M.; Murphy, R. C., Journal of the American Society for Mass Spectrometry 2007, $18(9), 1646-1652$.

46. Kim, S. H.; Shin, C. M.; Yoo, J. S., Rapid Communications in Mass Spectrometry 1998, 12 (11), 701-704.

47. Dekker, L. J. M.; van Kampen, J. J. A.; Reedijk, M. L.; Burgers, P. C.; Gruters, R. A.; Osterhaus, A. D. M. E.; Luider, T. M., Rapid Communications in Mass Spectrometry 2009, 23 (8), 1183-1188.

48. Caprioli, R. M.; Farmer, T. B.; Gile, J., Anal. Chem. 1997, 69 (23), 4751-60.

49. Kaspar, S.; Peukert, M.; Svatos, A.; Matros, A.; Mock, H.-P., Proteomics 2011, 11 (9), 1840-1850.

50. van Hove, E. R. A.; Smith, D. F.; Heeren, R. M. A., Journal of Chromatography A 2010, 1217 (25), 3946-3954.

51. Takats, Z.; Wiseman, J. M.; Gologan, B.; Cooks, R. G., Science 2004, 306 (5695), 471473.

52. Nemes, P.; Woods, A. S.; Vertes, A., Analytical Chemistry 2010, 82 (3), 982-988.

53. Nemes, P.; Barton, A. A.; Li, Y.; Vertes, A., Analytical Chemistry 2008, 80 (12), $4575-$ 4582. 
54. Nemes, P.; Barton, A.; Vertes, A., Analytical Chemistry 2009, 81 (16), 6668-6675.

55. Spengler, B.; Hubert, M., J. Am. Soc. Mass Spectrom. 2002, 13 (6), 735-48.

56. Chaurand, P.; Schriver, K. E.; Caprioli, R. M., J. Mass Spectrom. 2007, 42 (4), 476-89.

57. Cha, S.; Song, Z.; Nikolau, B.; Yeung, E., Analytical Chemistry 2009, 81 (8), 2991-3000.

58. Qiao, H.; Piyadasa, G.; Spicer, V.; Ens, W., Int. J. Mass Spectrom. 2009, 281 (1-2), 4151.

59. Cha, S.; Zhang, H.; Ilarslan, H.; Wurtele, E.; Brachova, L.; Nikolau, B.; Yeung, E., Plant Journal 2008, 55 (2), 348-360. 


\title{
CHAPTER 2. HIGH-SPATIAL AND HIGH-MASS RESOLUTION IMAGING OF SURFACE METABOLITES OF ARABIDOPSIS THALIANA BY LASER DESORPTION-IONIZATION MASS SPECTROMETRY USING COLLOIDAL SILVER
}

\author{
A paper published in Analytical Chemistry* \\ Ji Hyun Jun, Zhihong Song, Zhenjiu Liu, Basil J. Nikolau, Edward S. Yeung, and Young Jin Lee
}

\begin{abstract}
High-spatial and high-mass resolution techniques are developed and adopted for mass spectrometric imaging of surface metabolites on the model system, Arabidopsis thaliana which has contributed to the striking advances in plant molecular biology ${ }^{1}$ and been the most highly understood $^{2}$. For achieving the high-spatial resolution, the laser beam size was reduced by utilizing the optical fiber with small core diameter in a vacuum matrix-assisted laser desorption ionization-linear ion trap (vMALDI-LTQ) mass spectrometer and the matrix application was greatly improved using oscillating capillary nebulizer. As a result, single cell level spatial resolution of $\sim 12 \mu \mathrm{m}$ was achieved. Based on these improvements meeting the criteria for high spatial resolution, MS imaging of single cell level spatial resolution was directly applied to a
\end{abstract}

\footnotetext{
* Reprint with permission from Analytical Chemistry 2010, 82(8), 3255-3265 Copyright (C) 2010 American Chemical Society
} 
whole Arabidopsis flower and the substructures of an anther and single pollen grains at the stigma and anther were visualized. MS imaging at high-mass resolution was also achieved by utilizing the LTQ-Orbitrap mass spectrometer. High-mass resolution enables the differentiation of isobaric silver ion adducts having the same nominal mass such as C29 alkane $(\mathrm{m} / \mathrm{z} 515.3741)$ and C28 aldehyde ( $\mathrm{m} / \mathrm{z}$ 515.3377), and therefore the separate chemical images were able to be constructed for each of them. This could not be previously done with only the low mass resolution LTQ. From the benefits of high mass resolving capability, direct identification of the surface metabolites on the root was successfully performed within the mass error of $3 \mathrm{ppm}$. MS imaging of secondary roots at high spatial resolution visualized a high degree of localization of those metabolites and this was the first MS imaging on the root for molecular species.

\section{Introduction}

Mass spectrometric imaging (MSI) is not only able to profile the existing analytes on the biological tissue but also give the information about their spatial localization with abundances, which discloses the underlying molecular biology ${ }^{3-9}$. Among the ionization methods for imaging, secondary ion mass spectrometry (SIMS) that utilizes the ion beam to induce the ejection of secondary ions from the sample allows the submicrometer spatial resolution, but the extensive fragmentation of molecular species is often inevitable due to the energetic ion beams, Also, too small area sputtered with focused ion beams results in the limited sensitivity of detecting molecular species ${ }^{10}$. On the other hands, the ambient ionization method such as desorption electrospray ionization (DESI) allows the softer ionization, and the direct analysis and imaging of biological tissues can be performed. However, the typical lateral resolution achievable with this method is only $\sim 150-250 \mu \mathrm{m}^{11,12}$. For these reasons, matrix-assisted laser desorption 
ionization (MALDI) method that utilizes the laser to irradiate the sample tissues is a good method to obtain the chemical images of the analytes constituting the sample at a cellular or subcellular spatial resolution.

In MALDI, the matrix induces the efficient transfer of the laser energy to the sample, hence enhances the ionization of analytes in the sample. This method provides the soft desorption process so minimizes the fragmentation of molecular species being ionized ${ }^{13}$. However, conventional low-mass matrixes such as $\alpha$-cyano-4-hydroxycinnamic acid (CHCA) or 2,5dihydroxybenzoic acid (DHB) are well known for their unsuitability to analyze the small molecules because the peaks arising from the matrix interferes the detection of small molecules $(<500 \mathrm{Da})$ in the low $\mathrm{m} / \mathrm{z}$ regions ${ }^{14}$. The size of matrix crystals formed on the sample tissues is a crucial factor to be taken into account to achieve the MS imaging in high spatial resolution ${ }^{15}$. To overcome these problems associated with matrix, the use of water as a matrix in infrared laser ablation and/or ionization ${ }^{16}$ and the use of colloidal silver ${ }^{8}$ or graphite $^{2}$ as a matrix have been tried in MS imaging. Amongst these solutions, colloidal silver is more advantageous over the others for detection of epicuticular lipid metabolites on plant surface for several reasons. First, it does not crystalize, which allows good reproducibility of mass spectral signals and forms a homogeneous layer on the hydrophobic plant surfaces ${ }^{8}$. Secondly, silver ions form adducts with nonpolar or partially polar lipid molecules. Analysis of hydrocarbons with silver ions as a cationizing reagent has been previously reported ${ }^{17,18}$.

Reduction in laser beam size in combination with homogeneous matrix application is also needed to acquire the MS image in high spatial resolution and there have been many efforts for this including the use of a pinhole ${ }^{3}$ or utilization of multiple focusing lenses ${ }^{19}$. Recently, Caprioli's group used coaxial laser illumination to achieve high spatial resolution in the MS 
imaging ${ }^{20}$ and the spatial resolution of $4.8 \mu \mathrm{m}$ was obtained in the MS imaging on the mouse brain ${ }^{21}$. Achievement of the MS imaging in single cell level $(\sim 10 \mu \mathrm{m})$ using the smartbeam technology in the Ultraflex III time-of-flight (TOF) mass spectrometer has been also reported, in which the distribution of UV-absorbing secondary metabolites in plant tissues were visualized with no matrix ${ }^{22}$. A high resolution MS imaging has been previously performed on single crystals formed by standard analyte samples mixed with matrix on the target plate ${ }^{23}$ using the optical fiber, a convenient way to reduce the laser beam size. Metabolic analysis of single cells at a resolution of 30-40 $\mu \mathrm{m}$ using sharpened tip of optical fiber has been also reported in laserablation electrospray ionization (LAESI), but this work was only limited to profiling not imaging ${ }^{24}$. Previously, we performed MS imaging with a laser beam size of $\sim 100 \mu \mathrm{m}$ to reveal the distribution of epicuticular lipid metabolites on the various organs of plant utilizing colloidal silver as a matrix ${ }^{8}$. In this current study, we utilized an optical fiber with a $25 \mu \mathrm{m}$ core diameter to reach the single cell level spatial resolution and MS imaging of Arabidopsis flowers and roots were successfully accomplished in a spatial resolution of $\sim 12 \mu \mathrm{m}$.

In MS imaging, it is very beneficial to have a high mass resolution in terms of differentiating isobaric ions and conducting the direct chemical composition analysis of unknown species. To avoid the false analyte assignment, gas chromatography/mass spectrometry (GC/MS) analysis of surface extracted lipid metabolites is typically performed in parallel to MS imaging in our work. However, this strategy has a limitation that the identification of metabolites depends on the alternative identification method. Secondly, when isobaric ions were detected by GC/MS, the MS image constructed from the detected mass-to-charge $(\mathrm{m} / \mathrm{z})$ ratio cannot specify the different spatial distribution for each ion. Alternatively, tandem mass spectrometry (MS/MS) imaging (often called imaging mass spectrometry/mass spectrometry (IMS/MS)) can be performed to 
differentiate the isobaric ions, but the information on the fragmentation pattern is necessary ${ }^{25}$. Thirdly, the sampling depth is not the same in MS imaging and GC/MS. Adoption of highresolution mass spectrometry can not only differentiate isobaric ions by their slight mass difference but also identify the unknown species via an assignment of its chemical composition. Landgraf and co-workers have reported the utility of the high-resolution LTQ-Orbitrap mass spectrometer in MS imaging of rat brain spinal cord tissues ${ }^{26}$. Here, the differentiation of notorious isobaric epicuticular lipids, alkanes and aldehydes, on the plant surface and acquirement of separate image for each were successfully done by MALDI LTQ-Orbitrap. Direct identification of surface metabolites was also executed on the root.

\section{Experimental Section}

Chemicals. Colloidal silver (99.99\% pure silver, $0.65 \mathrm{~nm} ; 20 \mathrm{ppm}$ ) was obtained from Purest Colloids, Inc. (Westampton, NJ). Colloidal graphite aerosol spray was purchased from Alfa Aesar (Ward Hill, MA). 2-Propanol (HPLC grade) was purchased from Fisher Scientific (Fairlawn, NJ). N,O-Bis (trimethylsilyl)triflyoroacetamide with trimethylchlorosilane (BSTFA/TMCS), Murashige and Skoog basal salt mixture, and standard compound docosane were purchased from Sigma-Aldrich (St. Louis, MO).

Plant Growth Conditions. Arabidopsis thaliana ecotype Landsberg erecta (Ler-0) and eceriferum mutant (cer1, genetic stock CS31) were obtained from the Arabidopsis Biological Resource Center (Columbus, OH, US). Seeds were sterilized and sown on Murashige and Skoog basal salt mixture (MS) media in Petri dishes. The dishes were set in the growth room for 10 days after breaking seed dormancy by maintaining them at $4{ }^{\circ} \mathrm{C}$ for 4 days. On the 15 th day, the seedlings were transferred to soil in $5 \mathrm{~cm} \times 5 \mathrm{~cm}$ pots for continuous growth. Flower and root 
samples for imaging were collected on the 42 nd day and the 46th day, respectively. The stems used for the differentiation of isobaric ions with high-resolution mass spectrometry and the depth-profiling experiments were collected on the 38th and 29th day, respectively. The growth room was set at $24{ }^{\circ} \mathrm{C}$ with continuous illumination at $85 \mu \mathrm{E} \mathrm{m}^{-2} \mathrm{~s}^{-1}$ and ambient relative humidity. For the identification of root surface metabolites by GC/MS and LTQ-Orbitrap, seeds were sterilized and cultured in liquid media for 16 and 21 days, respectively, at room temperature with continuous shaking at $100 \mathrm{rpm}$ in $500 \mathrm{~mL}$ flasks containing $250 \mathrm{~mL}$ of MS media.

Sample Preparation for LDI MS Imaging. Each plant organ dissected from the intact plants was immediately attached to a stainless steel target plate using a conductive double-sided tape (3M; St. Paul, MN). To avoid physical contacts and damage on the sample tissues, a stream of nitrogen gas was used to attach the sample tissues onto the target plate. The attached tissues underwent drying step under a moderate vacuum ( $\sim 50$ Torr) for 30-60 min to minimize metabolite turnover.

An oscillating spray device ${ }^{27}$ that was modified from a commercial airbrush (Aztek A470; Testor; Rockford, IL) by replacing the inner spraying tip with a fused silica capillary (100 $\mu \mathrm{m}$ i.d. $360 \mu$ m o.d.; Polymicro Technology; Phoenix, AZ) was utilized for homogeneous matrix application. Matrixes were transferred to the oscillating capillary device via a syringe pump (Fisher Scientific; Pittsburgh, PA) and sprayed on plant tissues by nebulizing with $\mathrm{N}_{2}$ gas at a pressure of $40 \mathrm{psi}$. The distance between the tip of the capillary and the target plate was $9 \mathrm{~cm}$. Total spraying volumes were 0.15 and $0.3 \mathrm{~mL}$ for colloidal silver and colloidal graphite, respectively and the flow rate of matrix managed by syringe pump was $50 \mu \mathrm{L} \mathrm{min}^{-1}$. Each 
spraying of matrix for $12 \mathrm{~s}$ was followed by the suspension of spray for $30 \mathrm{~s}$ intervals to allow the vaporization of the solvent and thus reduce the aggregation of the matrix droplets.

Sample Preparation for Gas Chromatography/Mass Spectrometry. Root samples from liquid MS media were collected on the 16th day and weighed. An aliquot of internal standard (docosane) was added to the root surface. Surface lipids were extracted by completely immersing the roots in chloroform for $60 \mathrm{~s}$ and then dried under nitrogen gas. Samples were derivatized using BSTFA/TCMS $\left(65^{\circ} \mathrm{C}, 30 \mathrm{~min}\right)$ for $\mathrm{GC} / \mathrm{MS}$ analysis.

Mass Spectrometry Imaging. MS images of high spatial resolution were acquired with a linear ion trap mass spectrometry with a MALDI ion source (vMALDI LTQ; Thermo Electron; San Jose, CA) where a source pressure was kept at $170 \mathrm{mTorr}^{28}$. For the vMALDI LTQ, the $\mathrm{N}_{2}$ laser from Spectral Physics was used with a maximum energy of $280 \mu \mathrm{J} /$ pulse and maximum frequency of $20 \mathrm{~Hz}$. An optical fiber was used to deliver the laser to the mass spectrometer from the laser source. To reduce the laser beam size and increase the spatial resolution of MS imaging, the original optical fiber of $200 \mu \mathrm{m}$ i.d. was replaced with a $25 \mu \mathrm{m}$ core multimode optical fiber (Oz Optics; Ottawa, ON, Canada). The final laser spot size was measured as $\sim 12 \mu \mathrm{m}$ after the refocusing lens from a burn mark on a thin film of $\alpha$-cyano-4-hydroxycinnamic acid coated on a stainless steel plate.

A high-resolution mass spectrometer with a MALDI source (MALDI LTQ-Orbitrap Discovery; Thermo Electron) where a pressure was kept at 75-80 mTorr was also utilized in this work $^{28}$. It has a mass resolution of 30000 at $\mathrm{m} / \mathrm{z} 400$ and a mass accuracy of $5 \mathrm{ppm}$ with external calibration and 3 ppm with internal calibration. The $\mathrm{N}_{2}$ laser (MNL 100; Lasertechnik Berlin; Berlin, Germany) was also used in this system and it has a maximum energy of $80 \mu \mathrm{J}$ /pulse and maximum repetition rate of $60 \mathrm{~Hz}$. The laser was attached to the mass spectrometer 
and the laser beam was transferred through mirrors and lenses instead of an optical fiber. A black-painted stainless steel plate with a pinhole aperture with a diameter of $3 \mathrm{~mm}$ was placed in the laser beam path to reduce the laser spot size to $25 \mu \mathrm{m}$.

Data Acquisition and Processing for Mass Spectrometric Imaging. Data were acquired from plant tissues with a fixed number of laser shots without automatic gain control (AGC). The number of laser shots and laser power were optimized based on tests conducted on the small area of a test sample tissue with AGC turned-on. The sample was rastered with a step size of $12 \mu \mathrm{m}$ for high-spatial resolution MS imaging with the vMALDI LTQ and 25 or $50 \mu \mathrm{m}$ for high mass resolution MS imaging with MALDI LTQ-Orbitrap. A tissue imaging visualization software (ImageQuest 1.0.1; Thermo Finnigan) was used to generate the mass spectrometric images. The MS images for the compound of interest were constructed by normalizing the ion signals of interest with respect to the silver dimer peak intensity $\left(\mathrm{m} / \mathrm{z} 216 ;\left[{ }^{107} \mathrm{Ag}+{ }^{109} \mathrm{Ag}\right]^{+}\right)$on each spectrum and the mass windows of \pm 0.5 and \pm 0.008 Da were used for LTQ and LTQ-Orbitrap data, respectively. For the cases where data sets could not be properly displayed as MS images with ImageQuest, ion signals were extracted for each pixel using a custom program provided by Thermo (vMALDIDataExtract) and MS images were constructed using a homemade program written in Python (version 2.5; http://www.python.org/) with a Matplot library (http://matplotlibsourceforge.net/).

Gas Chromatography/Mass Spectrometry. GC/MS analysis was performed with an Agilent 6890 gas chromatograph and 5973N mass spectrometer (Agilent Technologies; Palo Alto, CA). The HP-5 ms column $(30 \mathrm{~m} \times 0.25 \mathrm{~mm}$ i.d. coated with $0.25 \mu \mathrm{m}$ thick (5\%-phenyl)methylpolysiloxane; Agilent Technologies) was temperature programmed from 80 to $320{ }^{\circ} \mathrm{C}$ at $5{ }^{\circ} \mathrm{C} \mathrm{min}^{-1}$ with helium flow rate at $2.0 \mathrm{~mL} \mathrm{~min}{ }^{-1}$. Electron ionization was used with $70 \mathrm{eV}$ 
electron energy. The GC/MS data files were deconvoluted by NIST AMDIS software and searched against an in-house mass spectral library and NIST05 spectral library.

\section{Results and Discussion}

Laser Spot Size and Matrix Homogeneity. The vMALDI LTQ mass spectrometer utilizes an optical fiber to deliver the laser beam from the laser source to the target plate inside the MALDI ion source. The laser spot size could be routinely reduced to about half the size of the optical fibers' core by utilizing the optical fibers with small core diameter and carefully realigning the optical components. Figure S-1 in the Supporting Information shows the laser burn marks of a size of $12-13 \mu \mathrm{m}$ with a $25 \mu \mathrm{m}$ optical fiber and $\sim 6 \mu \mathrm{m}$ with a $10 \mu \mathrm{m}$ optical fiber. However, the total laser output coming out of the optical fibers rapidly decreases with the decrease in the size of the optical fiber and as would be expected so does the subsequent ion signal. Therefore, to maintain the comparable ion signal, it was necessary to increase the laser power. For instance, the laser beam power was increased from 3 to $5 \%$ which was used with the $200 \mu \mathrm{m}$ optical fiber to almost $100 \%$ when a $25 \mu \mathrm{m}$ size optical fiber was used to reduce the laser spot size. Having maximized the laser beam power with a $25 \mu \mathrm{m}$ size optical fiber, it was rationalized that it may be possible to obtain equal quality LDI MS spectra even with a low laser power by acquiring and integrating spectra from multiple laser shots if ion yields are linearly proportional to the laser beam power. Based on this rationale, the use of optical fibers with even smaller diameters could be allowed. This could be performed with an ion trap mass spectrometer by accumulating a sufficient number of ions in the trap before mass spectral data acquisition. However, it should be noted that a minimum threshold laser power is required for surface

analytes to gain sufficient energy to be desorbed and ionized ${ }^{29}$. Ion yield increases linearly with 
respect to photon influx beyond the minimum threshold of laser power until it is saturated ${ }^{29}$. With the $10 \mu \mathrm{m}$ size optical fiber, the sufficient analyte ions could not be acquired for reliable MS imaging even though we could observe laser burn marks on the matrix with multiple laser shots (Figure S-1 in the Supporting Information). Thus, the practical limit of spatial resolution is $\sim 12 \mu \mathrm{m}$ by utilizing the optical fiber of $25 \mu \mathrm{m}$. It is expected to reduce the laser beam size further by a more powerful laser or a better way of coupling while maintaining the reliable signal levels. However, the reduction in laser spot size may lead to the MS imaging of only highabundance molecules since the sampling area is reduced as the square of the beam size and then the ion signals will be limited by the available analytes.

Another important factor determining the spatial resolution in MS imaging is the homogeneous application of the matrix. Colloidal silver was previously adopted as an additive for MS imaging of lipid metabolites on plant surfaces ${ }^{8}$. Figure 1a shows an SEM image of a bare stainless steel plate sprayed with colloidal silver using the commercial nebulizer previously used $^{8}$. It shows that the surface is covered with randomly spread $\sim 50 \mu \mathrm{m}$-size dark specks. According to Auger spectroscopic analysis, these dark specks have a high carbon content and it is presumed that these are originated from the organic additives which are added to the colloid preparations to minimize the aggregation of the silver nanoparticles. To minimize the heterogeneity caused by this, we adopted the use of oscillating capillary nebulizer developed by Sullards and co-workers ${ }^{27}$ and applied the matrix with a modified commercial airbrush. In detail, the original spray tip of a commercial airbrush was replaced with a fused silica capillary with $100 \mu \mathrm{m}$ i.d., which is connected to a syringe pump. The nebulizing gas flowing between the capillary and the outer tip helps reduce sizes of the droplets that are deposited on the surface by continuously oscillating the fused silica capillary. With this modification, sprayed droplets are 
almost invisible and the sizes of the organic, additive-derived black specks become much smaller, $\sim 10 \mu \mathrm{m}$ (Figure 1b). The size of black specks could be further reduced to 3-5 $\mu \mathrm{m}$ by adding four volumes of 2-propanol to the colloidal silver and spraying this solution using oscillating capillary nebulizer (Figure 1c). The inclusion of this organic solvent allows smaller droplets due to low surface tension and fast vaporization. Although the redistribution of surface analytes is potentially caused by the use of organic solvents for matrix dissolution in MALDI imaging ${ }^{27}$, the small droplet size would not invoke such an issue here. Figure 1d shows a zoomed-in image of one of the black specks shown in Figure 1c. White silver particles deposited on the surface have a size of $\sim 10 \mathrm{~nm}$ or smaller and homogeneously distributed on the outside of the speck, whereas those on the inside are somewhat aggregated toward the center. We suppose some silver particles get trapped by organic droplets and aggregate at the center as they are sprayed. Based on this observation, the homogeneity of matrix is limited by the size of the black specks, $3-5 \mu \mathrm{m}$ when applied with the addition of 2-propanol or $\sim 10 \mu \mathrm{m}$ when applied without 2-propanol. In either case, the homogeneity of matrix is sufficient to be used in the imaging experiments with the laser spot size achieved by the optical fiber.

Besides the laser beam size and matrix homogeneity that most influences the spatial resolution in MALDI MS imaging, there are also other factors that needs to be considered such as plate movement reproducibility and the homogeneity in analytes prepared on the surface. However, in the current study we simply hypothesize other factors are relatively ignorable compared to the laser beam size and assume our spatial resolution is mostly dictated by the laser spot size of $\sim 12 \mu \mathrm{m}$. To support this hypothesis, plate movement reproducibility is less than 3 $\mu \mathrm{m}$ according to the manufacturer's specification and homogeneous LDI MS images were 
obtained for standard compounds with a larger laser beam size (data not shown), so other factors can be considered ignorable.

MS Imaging of Arabidopsis Flowers at High Spatial Resolution. The chemical image of C29 alkane monitored as the silver ion adduct on an Arabidopsis flower is shown in Figure 2. This image was obtained at a spatial resolution of $12 \mu \mathrm{m}$. "Photo-quality" fine chemical images were successfully obtained for surface lipid metabolites in this small area of only $4 \times 3 \mathrm{~mm}^{2}$. Such spatial resolution allows the localization of the molecules at the single cell level, visualizing the fine structures of an anther (tip of stamen) and single pollen grains collected at the stigma (tip of carpel) and anthers as shown in the images with higher magnification in Figure 2. Several strong lines of evidence prove that those single pixels with high ion intensities around the stigma and anthers are pollen grains: (1) the size is equivalent to the known size of Arabidopsis pollens, $\sim 10 \mu \mathrm{m}$; (2) they are found only at the tip of stamens (male organ) and carpel (female organ), where pollens are supposed to be found; (3) the similar mass spectral features are observed on each single pollen pixel (data not shown). These pollen grains probably fell to the MALDI plate and were scattered around the stigma and anthers during the sample preparation. The secondary ion mass spectrometry (SIMS) technique has been used by Nakata and co-workers to obtain cross-sectional images of large size pollen particles $(\sim 100 \mu \mathrm{m})$, but mostly fragments were detected such as $\mathrm{PO}_{3}{ }^{-}$or $\mathrm{HPO}_{4}{ }^{-30}$.

Figure 3 shows "single pixel" mass spectra derived from different sub-organs of the flower shown in Figure 2: single pollen grain, side of stamen filament, side of style (middle part of carpel), tip of sepal, middle of sepal, and side of petal. The signal intensity of each lipid molecule is normalized to the silver dimer peak, $\left[{ }^{107} \mathrm{Ag}+{ }^{109} \mathrm{Ag}\right]^{+}(\mathrm{m} / z$ 216) for semiquantitative comparison (Table 1). There are a few things to be noted. First of all, ion signal in each pixel is 
sufficient although the dimension of each pixel is only $12 \times 12 \mu \mathrm{m}^{2}$. Ion signal levels of the base peaks are $\sim 10^{5}$ counts in all the spectra shown in Figure 3, implying that the reduction in sampling size to $2-5 \mu \mathrm{m}$ also results in sufficient signals. Secondly, each sub-organ of the flower contains unique metabolite distributions based on the fact that spectral features seen on each suborgan are quite different. The pollen grain has a broad distribution of epicuticular lipid molecules (Figure 3A; Table 1). In contrast, the carpel has C29 ketone as the most abundant surface lipid, showing more than 5-fold higher intensity than on the other tissues. C29 alkane and C30 fatty acid are also the most abundant among the tissues (Figure 3B; Table 1). The fact that they are intermediates on the same metabolic pathway ${ }^{31}$ (C30 fatty acid $\rightarrow(\mathrm{C} 30$ aldehyde $) \rightarrow \mathrm{C} 29$ alkane $\rightarrow \mathrm{C} 29$ ketone) suggests that fatty acid elongation to C30 fatty acid and the subsequent production of the downstream metabolites is especially efficient in the epidermal cells of the carpel. Another result to support this hypothesis is the finding that carpels have the most abundant level of $\mathrm{C} 30$ alcohol, than on the other tissues, which is an alternative metabolic product of the C30 fatty acid. In contrast, at the tip of sepal (Figure 3D; Table 1), C29 alkane is the second highest abundant epicuticular lipid, whereas the abundance of C29 ketone is the lowest. This result may indicate that the conversion of C29 alkane to C29 ketone is much less efficient at the tip of the sepal. On the other hand, the mass spectra acquired from the middle of the sepal (Figure 3E; Table 1) indicates a very low abundance of epicuticular lipid compounds overall, suggesting that at the microscale this biosynthetic pathway is developmentally regulated through sepal development, so that epicuticular lipid accumulation increases as the sepal expands from its base. At this point, we want to point out that most sepal tissues samples in Figure 2 were on the inner side of the sepal and inside the flower bud prior to flower opening (the outer side of the sepal was in contact with the sample plate and could not be sampled in these experiments). 
Our findings may indicate that the accumulation of the epicuticular lipids is induced when the flower opens, and hence these tissues are exposed to a drier environment, consistent with the functionality of this material as water-barrier ${ }^{32}$. The tip of sepal is partially exposed to air, to more desiccating conditions, and therefore these lipids function to inhibit water-loss, which explains the higher abundance of epicuticular lipids at the tip of sepal. In the case of the stamen filament and petal (C, F) they show an average abundance level of epicuticular lipids compared to other flower tissues. The biochemical implication suggested above is our best speculation and subject to further verification; however, it is noteworthy that our technology has revealed a new discovery of a high degree of localization of lipid metabolites. This has not been previously possible and could lead to novel hypotheses. We previously compared the relative abundances of epicuticular lipid molecules on the surface of carpel, sepal, and petal but at a lower spatial resolution $(\sim 100 \mu \mathrm{m})^{8}$. The distribution of these metabolites could be compared among fine locations in each tissues such as stamen filament, tip of sepal, and pollen grains (Table 1) at the higher spatial resolution afforded by the modifications described herein. The relative abundances of each lipid metabolite that were evaluated on a single pixel of carpel, sepal, and petal are similar to the data previously reported ${ }^{8}$, demonstrating the consistency of the method and the underlying biology.

It is necessary to carefully consider the ionization efficiencies of the individual lipid metabolites and their changes on each tissue during the interpretation of the data generated via this method. In the discussion above, it is assumed that the ion signals can be directly correlated with their abundances when we made semiquantitative comparisons of the lipids on various parts of flower tissues. Yost's group showed that matrix effects in MALDI ionization necessitate the need for internal standards for accurate quantification in MS imaging ${ }^{33}$. In fact, ion yields 
depend on many variables such as desorption efficiency, ionization efficiency, and instrument transmission efficiency. The last factor is relatively minor because they are typically mass dependent and the lipid molecules of interest in this study are within the narrow mass range of $m / z 470-560$.

Each metabolite could have different ionization efficiency. For example, in LDI MS, the ion signals of alkane are 10-100 times less than other epicuticular lipid molecules according to inparallel analyses with GC/MS ${ }^{8}$. Although ion abundances of different lipid molecules cannot be directly compared to each other, it is possible to compare the relative abundance of the same metabolite between different organs and tissues. In LDI with colloidal silver does not involve cocrystallization of the anlaytes with the matrix unlike MALDI, and it is unlikely that the adduct formation would incur at the same time with desorption process considering silver nanoparticles are sporadically scattered (Figure 1d). Therefore, the ionization of epicuticular lipid metabolites as silver ion adducts seems to occur through a two step process, desorption from the surface and adduct formation in the gas phase. Because the adduct formation occurs after desorption from the surface, it would not be affected by the matrix. However, desorption and ionization of the silver atoms could have a strong matrix effect, which in turn could affect silver ion adduct formation. This effect can be minimized by normalizing the ion current to the silver dimer peak. Desorption efficiency of each analyte can also be affected by the matrix, but its effect is expected to be relatively small. The van der Waals interaction of lipid molecules with cuticles of which most plant surfaces consist should not be significantly different among the tissues. Therefore, the rationale to discuss concerning the semiquantiative comparison of each metabolite between organs and tissues can be valid. 
Figure 4 shows MS images of additional metabolites, specifically C29 ketone and C26 fatty acid. As can be seen in Figure 3 and Table 1, C29 ketone is mostly concentrated on the carpel, while C26 fatty acid is abundant on the petal, stamen, and tip of the sepal. A magnified image of the upper part of a stamen filament in Figure 2 is shown in Figure 5A. At the $X$ position indicated by the arrow, ion abundance profiles were taken along a series of single pixels vertically from the bottom of the image for the $Y$ coordinates between 2400 and $3000 \mu \mathrm{m}$ from the base of the image (Figure 2). Figure 5B shows the ion abundance profiles of C29 alkane, C29 ketone, and C26 fatty acid. It is noteworthy to point out that the ion signals are periodically fluctuated every one or two pixels of high abundance alternating with one of low abundance. This probably indicates cell-to-cell ion abundance variation which matches with the longitudinal epidermal cell size of $\sim 30 \mu \mathrm{m}$ along an Arabidopsis stamen filament ${ }^{34}$. As shown in Figure S-2 in the Supporting Information, similar profiles were obtained in a series of pixels nearby. The pixels of high abundance may be from the center of epidermal cells and those of low abundance may be aligned with the cell junctions. This supports that the spatial resolution of the technology described herein allows the mapping of the distribution of metabolites at the single-cell level.

High-Resolution MS Imaging of Roots. MS imaging has been applied to Arabidopsis roots to further exploit this high spatial resolution technology. The chemical image of C18 fatty alcohol caffeate ester on an Arabidopsis root surface obtained with $12 \mu \mathrm{m}$ spatial resolution is shown in Figure 6. The fine chemical images of root structures are visualized. Spengler and Huber have performed submicrometer MS imaging of potassium and calcium on pine tree roots ${ }^{19}$, but this is the first MS imaging of secondary roots for molecular species. The chemical compounds detected on the root surface are different from those of epicuticular lipid metabolites on other plant surfaces. For example, C18 fatty alcohol caffeate and C20 fatty alcohol coumarate 
esters are observed on the Arabidopsis root. The high spatial resolution allowed us to study and image these metabolites directly on the root surface.

A number of difficulties were encountered associated with imaging root samples during the sample preparation for MS imaging. Specifically, root samples are not flat and difficult to be attached flat to the surface, which in turn causes some parts of root fibers are not in depth of field of the laser not getting the sufficient laser power for LDI. In Figure 6, it is observed in MS image that some local areas do not have enough ion signals in comparison with the features visualized in the optical image. This problem did not occur with other plant organs such as flowers, leaves, or stems, which are more flexible and thin, and therefore can be easily attached to the plate flat. It should be noted that distorted laser depth of filed may affect the ion signals and ion signals may not represent the abundance of analyte present on the surface. Therefore, caution is needed in quantitative interpretation of this data.

High Mass Resolution in MS Imaging. The LTQ-Orbitrap Discovery with a MALDI source was adopted to acquire high-mass resolution spectra for metabolite imaging. This instrument provides less mass resolution than other high-resolution mass spectrometers, 30000 at $\mathrm{m} / \mathrm{z} 400$, but mass accuracy of less than 3 ppm could be still routinely attained with internal calibration. By utilizing the high mass resolution mass spectrometer, the isobaric ions having the same nominal $\mathrm{m} / \mathrm{z}$ values can be differentiated. For instance, alkanes and aldehydes, notorious isobaric ions, could not be previously differentiated in LDI-MS of plant metabolites using low-resolution mass spectrometer. Plant epicuticular aldehydes typically have even number of carbon atoms and alkanes have odd number of carbon atoms. Aldehydes have the same nominal mass as the alkane homologue with one additional carbon atom as the aldehyde functional group (CHO) has the same nominal mass as $\mathrm{CH}_{2} \mathrm{CH}_{3}$, or $29 \mathrm{Da}$ (exact mass difference is $0.0364 \mathrm{Da}$ ). The LDI-MS 
spectrum of epicuticular lipid metabolites on an Arabidopsis stem obtained with Orbitrap mass analyzer is shown in Figure 7 for the $m / z$ range 514-520. Although ${ }^{107} \mathrm{Ag}$ adducts of $\mathrm{C} 29$ alkane and C28 aldehyde have the same nominal mass of $\mathrm{m} / \mathrm{z} 515$, these isobaric ions could be clearly differentiated using high-resolution mass spectrometer. Chemical composition analysis exactly matches the experimental $\mathrm{m} / \mathrm{z}$ values within a mass error of $3 \mathrm{ppm}$. High-resolution MS is the best way to differentiate these isobaric ions since they have almost no fragmentation in typical ion trap CID. The separate MS images for C29 alkane and C28 aldehyde could be also constructed based on their differentiation using high-resolution MS (Figure 7). The isobaric ions having nominal mass of $\mathrm{m} / \mathrm{z} 517$ with an exact mass difference of $0.0203 \mathrm{Da},{ }^{109} \mathrm{Ag}$ adduct of C29 alkane $(m / z 517.3734)$ and ${ }^{107} \mathrm{Ag}$ adduct of $\mathrm{C} 28$ alcohol $(\mathrm{m} / \mathrm{z} 517.3531)$, could be also differentiated in the mass spectrum as shown in Figure 7. In the previous study with the ion trap mass spectrometer ${ }^{8}$, the GC/MS data inferred the presence of C28 alcohol and LDI-MS data allowed its quantification with the ${ }^{109} \mathrm{Ag}$ adduct ( $m / z$ 519), but its presence was clearly confirmed by both ${ }^{107} \mathrm{Ag}$ and ${ }^{109} \mathrm{Ag}$ adducts with high-resolution MS. High-resolution MS is very valuable to perform unambiguous identification of most metabolites directly from the sample surface without any separate analysis such as chromatographic separation or prior knowledge of the analytes in the sample. In addition, the MS/MS capability in the LTQ, of the LTQ-Orbitrap can further assist the validation.

As briefly cited above, the lipid metabolites detected on the root surface are quite different from typical epicuticular lipids on the surface of aerial organs. The lipid compounds rich in C18C22 alkyl esters of 4-hydroxycinnamic acids were detected from the chloroform extracts of Arabidopsis roots by GC/MS performed by Li and co-workers ${ }^{35}$, and we also detected these compounds in MS imaging of root surfaces (Figure 6). As shown in Table 2, C18-C22 alkyl 
esters of coumarate, caffeate, and ferulate were identified using MALDI LTQ-Orbitrap as major molecular species on the root surfaces, as has been observed by Li and co-workers, also with a C29:2 sterol. These metabolites were identified based on two criteria: (1) the match of their experimentally determined $\mathrm{m} / \mathrm{z}$ values with theoretical values, which are within $3 \mathrm{ppm}$; and (2) GC/MS analysis of chloroform-extract of roots. Table 2 shows the abundances represented by GC/MS analysis are mostly comparable to those by LDI LTQ-Orbitrap. It should be noted that GC/MS data, of both Li and co-workers' and ours, identified C28:1 and C29:1 sterols while LDIMS detected only C29:2 sterol. Based on the data provided by GC/MS analysis, the abundances of C28:1 and C29:1 sterols are much higher than C29:2 sterol and also their ionization efficiencies should not be significantly different from that of C29:2 sterol. Therefore, it is suspected that the differences by two methods may arise from the differences in the sampling depths between two methods.

Other surface lipids detected by $\mathrm{Li}$ and co-workers ${ }^{35}$ such as primary alcohols and fatty acids were also found in our GC/MS analysis (data not shown), but LDI-MS did not detect these molecules. This difference may arise from: (1) the shallow sampling depth in LDI-MS imaging compared to chloroform extraction for GC/MS analysis and (2) low abundance of these lipid molecules on roots as compared with other organs; 10-100 times less abundant according to GC/MS analysis (data not shown) ${ }^{8}$. Considering the fact that those lipids could be detected on flowers (Table 1 and Cha et al. ${ }^{8}$ ), leaves (Cha et al. ${ }^{8}$ ), and stems (data not shown), low ionization efficiency is an unlikely explanation. It should be mentioned that there are a few days of unintentional gap in harvesting Arabidopsis roots between those used for GC/MS and LDIMS; at the time of harvest, they are 16 and 21 days old, respectively. However, based on the 
overall consistency in GC/MS data between ours and Li and co-workers' in which 7 week-old roots were used, it is assumed that the gap in the age of roots did not affect the current result.

The optical fiber was not utilized to transfer the laser beam from the laser source to the target plate in the mass spectrometer for the MALDI LTQ-Orbitrap system. Instead of use of the optical fiber, two mirrors and a lens were used to couple the laser beam ${ }^{28}$ and the inherent spot size with the $\mathrm{N}_{2}$ laser is $\sim 50 \mu \mathrm{m}$ in its diameters. To achieve a smaller laser spot size for higher spatial resolution MS imaging, a stainless steel plate with a pinhole with an aperture diameter of $3 \mathrm{~mm}$ was placed in the laser beam path to mechanically reduce the beam size. The final laser spot size achieved with this modification was $\sim 25 \mu \mathrm{m}$. Although this does not provide fine images of roots as shown in Figure 6, the high quality mass spectra were obtained with high mass resolution for metabolite identifications as shown in Table 2 (image not shown).

Depth Resolved MS Imaging. Acquiring chemical information in the third dimension, in terms of depth, is challenging in MS imaging. 3D MS imaging was successfully demonstrated by Caprioli and co-workers by acquiring 2D MS images from multiple layers of tissue slices prepared from a rat and a mouse brain ${ }^{36,37}$. In the current study, an alternative approach was attempted to acquire depth profiling in submicrometer scales. Specifically, we adopted the concept of ion sputtering in surface analysis. In Auger electron spectroscopy or X-ray photoelectron spectroscopy, the surface is sputtered out, layer by layer, with ion beams in order to obtain atomic concentrations as a function of depth. In the similar context, the surface of plant tissues was sputtered out, layer by layer, with high-power laser beams to probe analytes at various depths.

To test the plausibility of this method, the following preliminary experiment was performed. First of all, a 2D MS image of the surface metabolites of an Arabidopsis stem sprayed with 
colloidal graphite was obtained and then the same surface area was scanned again with multiple shots of the high-power laser to sputter out the surface layer(s). Prior to the next image acquisition, the sample plate was ejected from the instrument for reapplication of the colloidal graphite matrix and reinserted into the mass spectrometer. The MS images of a few representative metabolites on the surface and deeper areas of the Arabidopsis stem are shown in Figure 8. Alkanes and ketones could not be detected in these analyses because colloidal graphite is not a good additive for ionizing nonpolar compounds. However, a number of very-long chain fatty acids (C24-C30) were detected in high abundance on the surface of the stem whereas kaempferol, a flavonoid, was not found in this layer. On the contrary, kaempferol was detected at much higher abundance in the deeper layer and fatty acid compounds were barely found. This observation is consistent with our expectation to detect the epicuticular lipid metabolites on the surface ${ }^{8}$ and kamepferol and its derivatives in the deeper layers because they are induced with exposure to higher light levels and accumulate in the deeper layers of the plant tissues ${ }^{38}$. All the MS images were generated with accurate mass from the data obtained with the Orbitrap for their chemical assignments with the enhanced confidence. It is plausible to construct the 3D MS images of metabolites by using the MS images in multiple layers obtained through the procedures described above, repeating laser sputtering, matrix application, and MS imageacquisition. To further demonstrate the proof of concept, more experiments need to be conducted for samples with well-defined multilayers.

One of the significant bottlenecks in this approach, however, is the very long time consumption for data acquisition. Also, the sputtering rate may not be homogenous throughout the surface, and therefore the exact information in terms of depth may not be inferred. Hence, a means of calibrating the depth of each layer that is being probed is in need. 


\section{Conclusions}

We obtained a spatial resolution of $\sim 12 \mu \mathrm{m}$ in MS imaging by utilizing an optical fiber with a $25 \mu \mathrm{m}$ core diameter, and by improving the matrix homogeneity through the use of modified oscillating capillary nebulizer device. The high spatial resolution achieved was successfully applied for MS imaging of lipid metabolites on the surface of Arabidopsis flowers and roots. MS imaging of a whole flower was achieved at the single cell level for the first time, and therefore fine features such as single pollen grains could be visualized and it was also possible to compare the profile of the surface lipid metabolites from single pixel mass spectra on various plant tissues. It was suggested that the subcellular MS imaging even at a spatial resolution down to a few micrometers may be possible based on the sufficient ion signals obtained at a spatial resolution of $\sim 12 \mu \mathrm{m}$ in the current study. The periodic fluctuation of the ion signal shown in its profiles along a series of single pixels on a stamen matches the size of the longitudinal epidermal cell and this supports our single cell level resolution. The high mass resolving capability of the Orbitrap mass analyzer used in this study enabled the differentiation of isobaric ions of aldehydes and alkanes and also direct identifications of surface metabolites on roots. The surface sputtering with high-power laser and the subsequent MS image-acquisition suggests the feasibility of 3D MS imaging.

In MS imaging with both high spatial and high mass resolution, the most significant practical limitation is data acquisition time. To be more specific, it takes less than an hour to acquire an ion trap MS image of a whole Arabidopsis flower with a size of $4 \times 3 \mathrm{~mm}^{2}$ at $50 \mu \mathrm{m}$ resolution but it takes $7-10 \mathrm{~h}$ at $12 \mu \mathrm{m}$ resolution due to the larger number of pixels to be scanned. If even a higher spatial resolution of $5 \mu \mathrm{m}$ is pursued, it will take over $40 \mathrm{~h}$ and it will take additional 3-5 
more times to acquire the mass spectra in the high-mass resolution mode. Depth resolved MS imaging described last may not be possible due to the long term instability of the laser beam and other experimental conditions. Imaging mass spectrometry in the microscope mode is an alternative way ${ }^{39}$, however, it suffers from the limited field of view. To overcome this bottleneck, further technical improvement is urgently in need, such as the recent development of a high speed time-of-flight mass spectrometer for MS imaging ${ }^{40}$.

\section{Acknowledgement}

Acknowledgement is made to Sangwon Cha for his technical advice and Jame W. Anderegg in the Ames Laboratory for the SEM images. We also acknowledge the technical expertise and advice of Ann Perera, W. M. Keck Metabolomics Research Laboratory. The Ames Laboratory is operated for the U.S. Department of Energy by Iowa State University under Contract No. DEAC02-07CH11358. This work was supported by U.S. Department of Energy, Office of Basic Energy Science, Division of Chemical Sciences.

\section{Appendixes}

Photos of laser burn marks obtained with optical fibers with different inner core diameter and single pixel ion abundance profiles for C29 alkane, C29 ketone, and C26 fatty acid along a series of single pixels at the different $X$ positions are listed in Appendix 1. 
Table 1. Semiquantification of Surface Metabolites on Single Pixel of Flower Tissues ${ }^{a}$

\begin{tabular}{|c|c|c|c|c|c|c|c|}
\hline \multirow[b]{2}{*}{$m / z$} & \multirow[b]{2}{*}{ Assignment } & \multicolumn{6}{|c|}{ Relative Intensity (\%) } \\
\hline & & $\begin{array}{c}\text { A } \\
\text { Pollen grain }\end{array}$ & $\begin{array}{c}\text { B } \\
\text { Side of carpel }\end{array}$ & $\begin{array}{c}\text { C } \\
\text { Stamen } \\
\text { filament }\end{array}$ & $\begin{array}{l}\text { D } \\
\text { Tip of sepal }\end{array}$ & $\begin{array}{c}\mathbf{E} \\
\text { Middle of } \\
\text { sepal }\end{array}$ & $\begin{array}{c}\mathbf{F} \\
\text { Side of petal }\end{array}$ \\
\hline 487 & {$\left[\mathrm{C} 27 \text { alkane }+{ }^{107} \mathrm{Ag}\right]^{+}$} & $25.2 \pm 4.3$ & $26.2 \pm 5.6$ & $28.3 \pm 11.2$ & $23.9 \pm 4.2$ & $19.4 \pm 7.4$ & $30.9 \pm 16.9$ \\
\hline 515 & {$\left[\mathrm{C} 29 \text { alkane }+{ }^{107} \mathrm{Ag}\right]^{+}$} & $66.6 \pm 4.2$ & $99.8 \pm 16.7$ & $41.6 \pm 3.0$ & $92.6 \pm 20.0$ & $25.2 \pm 11.1$ & $55.6 \pm 17.7$ \\
\hline 543 & {$\left[\mathrm{C} 31 \text { alkane }+{ }^{107} \mathrm{Ag}\right]^{+}$} & $35.0 \pm 5.9$ & $47.6 \pm 7.6$ & $13.2 \pm 2.2$ & $16.0 \pm 5.7$ & $9.2 \pm 6.7$ & $11.6 \pm 3.8$ \\
\hline 491 & {$\left[\mathrm{C} 26 \text { alcohol }+{ }^{109} \mathrm{Ag}\right]^{+}$} & $4.0 \pm 2.3$ & $6.4 \pm 4.3$ & $11.5 \pm 8.0$ & $3.8 \pm 2.4$ & $3.8 \pm 2.5$ & $3.0 \pm 3.3$ \\
\hline 519 & {$\left[\mathrm{C} 28 \text { alcohol }+{ }^{109} \mathrm{Ag}\right]^{+}$} & $8.5 \pm 2.8$ & $13.8 \pm 2.7$ & $7.7 \pm 1.0$ & $11.1 \pm 3.0$ & $6.2 \pm 1.3$ & $9.8 \pm 3.6$ \\
\hline 547 & {$\left[\mathrm{C} 30 \text { alcohol }+{ }^{109} \mathrm{Ag}\right]^{+}$} & $4.8 \pm 3.8$ & $12.9 \pm 4.7$ & $2.5 \pm 2.5$ & $2.3 \pm 0.5$ & $1.2 \pm 0.6$ & $1.4 \pm 0.8$ \\
\hline 475 & {$\left[\mathrm{C} 24 \text { fatty acid }+{ }^{107} \mathrm{Ag}\right]^{+}$} & $13.8 \pm 6.1$ & $15.7 \pm 6.3$ & $23.0 \pm 1.6$ & $14.8 \pm 2.2$ & $13.6 \pm 3.9$ & $40.7 \pm 24.0$ \\
\hline 503 & {$\left[\mathrm{C} 26 \text { fatty acid }+{ }^{107} \mathrm{Ag}\right]^{+}$} & $12.8 \pm 1.2$ & $10.2 \pm 0.4$ & $26.7 \pm 5.3$ & $12.3 \pm 4.2$ & $6.7 \pm 1.5$ & $22.9 \pm 6.1$ \\
\hline 559 & {$\left[\mathrm{C} 30 \text { fatty acid }+{ }^{107} \mathrm{Ag}\right]^{+}$} & $2.0 \pm 0.5$ & $19.4 \pm 3.3$ & $4.1 \pm 1.7$ & $7.9 \pm 1.9$ & $1.1 \pm 0.7$ & $12.7 \pm 8.0$ \\
\hline 529 & {$\left[\mathrm{C} 29 \text { ketone }+{ }^{107} \mathrm{Ag}\right]^{+}$} & $20.4 \pm 2.7$ & $119.7 \pm 16.1$ & $11.1 \pm 1.7$ & $5.6 \pm 2.9$ & $9.9 \pm 7.1$ & $8.8 \pm 3.2$ \\
\hline
\end{tabular}

${ }^{a}$ Relative intensities are ion signals normalized to silver dimer, $\left[{ }^{107} \mathrm{Ag}+{ }^{109} \mathrm{Ag}\right]^{+}(\mathrm{m} / \mathrm{z} 216)$. Standard deviation is obtained from multiple pixels near the positions for each tissue indicated in Figure 2. Noticeable ion signals discussed in the text are marked in bold. C28 fatty acid is not included because ${ }^{107} \mathrm{Ag}$ adduct $(\mathrm{m} / \mathrm{z} 531)$ is overlapping with isotopic contribution from C29 ketone, ([C29 ketone $\left.\left.+{ }^{107} \mathrm{Ag}\right]\right]^{+}$, and the ${ }^{109} \mathrm{Ag}$ adduct $(\mathrm{m} / \mathrm{z} 533)$ is overlapping with a matrix contaminant. ${ }^{109}$ Ag adduct were used for alcohols because the $\mathrm{m} / \mathrm{z}$ values of the ${ }^{107} \mathrm{Ag}$ adduct are overlapping with the ${ }^{109} \mathrm{Ag}$ adduct of alkanes. 
Table 2. Identification of Root Surface Metabolites Using the LTQ-Orbitrap ${ }^{a}$

\begin{tabular}{|c|c|c|c|c|c|}
\hline \multirow{2}{*}{ Ion Species } & \multicolumn{4}{|c|}{ MALDI LTQ-Orbitrap } & \multirow{2}{*}{$\begin{array}{l}\text { Wax load in } \\
\text { GC-MS } \\
(\text { nmol/g) }\end{array}$} \\
\hline & $\begin{array}{l}\text { Chemical } \\
\text { formula }\end{array}$ & Experimental $^{\mathrm{b}}{ }^{m / z}$ & Theoretical & $\begin{array}{c}\text { Relative } \\
\text { Intensity }(\%)^{c}\end{array}$ & \\
\hline$\left[\mathrm{C} 28: 1 \text { sterol }+{ }^{107} \mathrm{Ag}\right]^{+}$ & $\mathrm{C}_{28} \mathrm{H}_{48} \mathrm{O}^{107} \mathrm{Ag}$ & n.d. & 507.2751 & - & $50.1 \pm 9.1$ \\
\hline$\left[\mathrm{C} 29: 1 \text { sterol }+{ }^{107} \mathrm{Ag}\right]^{+}$ & $\mathrm{C}_{29} \mathrm{H}_{50} \mathrm{O}^{107} \mathrm{Ag}$ & n.d. & 521.2907 & - & $142.4 \pm 3.0$ \\
\hline$\left[\mathrm{C} 29: 2 \text { sterol }+{ }^{107} \mathrm{Ag}\right]^{+}$ & $\mathrm{C}_{29} \mathrm{H}_{48} \mathrm{O}^{107} \mathrm{Ag}$ & $519.2736 \pm 0.0015$ & 519.2751 & $0.8 \pm 0.4$ & $21.6 \pm 3.8$ \\
\hline$\left[\text { C18 coumarate }+{ }^{107} \mathrm{Ag}\right]^{+}$ & $\mathrm{C}_{27} \mathrm{H}_{44} \mathrm{O}_{3}{ }^{107} \mathrm{Ag}$ & $523.2335 \pm 0.0001$ & 523.2337 & $12.4 \pm 9.3$ & $87.2 \pm 2.2$ \\
\hline$\left[\mathrm{C} 20 \text { coumarate }+{ }^{107} \mathrm{Ag}\right]^{+}$ & $\mathrm{C}_{29} \mathrm{H}_{48} \mathrm{O}_{3}{ }^{107} \mathrm{Ag}$ & $551.2648 \pm 0.0001$ & 551.2650 & $8.7 \pm 5.0$ & $47.3 \pm 10.5$ \\
\hline$\left[\mathrm{C} 22 \text { coumarate }+{ }^{107} \mathrm{Ag}\right]^{+}$ & $\mathrm{C}_{31} \mathrm{H}_{52} \mathrm{O}_{3}{ }^{107} \mathrm{Ag}$ & $579.2961 \pm 0.0002$ & 579.2963 & $2.3 \pm 1.1$ & $10.0 \pm 3.7$ \\
\hline$\left[\mathrm{C} 18 \text { caffeate }+{ }^{107} \mathrm{Ag}\right]^{+}$ & $\mathrm{C}_{27} \mathrm{H}_{44} \mathrm{O}_{4}{ }^{107} \mathrm{Ag}$ & $539.2285 \pm 0.0003$ & 539.2286 & $3.1 \pm 3.2$ & $3.4 \pm 2.4$ \\
\hline$\left[\mathrm{C} 20 \text { caffeate }+{ }^{107} \mathrm{Ag}\right]^{+}$ & $\mathrm{C}_{29} \mathrm{H}_{48} \mathrm{O}_{4}{ }^{107} \mathrm{Ag}$ & $567.2596 \pm 0.0005$ & 567.2599 & $1.8 \pm 1.8$ & $0.7 \pm 0.7$ \\
\hline$\left[\mathrm{C} 22 \text { caffeate }+{ }^{107} \mathrm{Ag}\right]^{+}$ & $\mathrm{C}_{31} \mathrm{H}_{52} \mathrm{O}_{4}{ }^{107} \mathrm{Ag}$ & $595.2911 \pm 0.0004$ & 595.2912 & $1.4 \pm 0.9$ & $0.09 \pm 0.01$ \\
\hline$\left[\mathrm{C} 18 \text { ferulate }+{ }^{107} \mathrm{Ag}\right]^{+}$ & $\mathrm{C}_{28} \mathrm{H}_{46} \mathrm{O}_{4}{ }^{107} \mathrm{Ag}$ & $553.2450 \pm 0.0014$ & 553.2442 & $0.4 \pm 0.2$ & $2.7 \pm 1.5$ \\
\hline$\left[\mathrm{C} 20 \text { ferulate }+{ }^{107} \mathrm{Ag}\right]^{+}$ & $\mathrm{C}_{30} \mathrm{H}_{50} \mathrm{O}_{4}{ }^{107} \mathrm{Ag}$ & $581.2768 \pm 0.0011$ & 581.2755 & $1.8 \pm 0.5$ & $1.1 \pm 0.8$ \\
\hline$\left[\mathrm{C} 22 \text { ferulate }+{ }^{107} \mathrm{Ag}\right]^{+}$ & $\mathrm{C}_{32} \mathrm{H}_{54} \mathrm{O}_{4}{ }^{107} \mathrm{Ag}$ & $609.3056 \pm 0.0010$ & 609.3068 & $0.5 \pm 0.6$ & $0.1 \pm 0.05$ \\
\hline
\end{tabular}

${ }^{a}$ Data from the cer 1 mutant of Arabidopsis thaliana were shown. ${ }^{b}$ Data were taken from a few representative pixels in root imaging obtained with $25 \mu \mathrm{m}$ spatial resolution. N.d.: Not detected. ${ }^{c}$ Relative intensities were obtained by normalizing ion intensities to the silver dimer, $\left.\left[{ }^{107} \mathrm{Ag}+{ }^{109} \mathrm{Ag}\right]\right]^{+}(\mathrm{m} / \mathrm{z} 216) .{ }^{d}$ Wax load is obtained from three replicates of GC/MS experiments. 


\section{References}

1. Meissner, R.; Jacobson, Y.; Melamed, S.; Levyatuv, S.; Shalev, G.; Ashri, A.; Elkind, Y.; Levy, A., Plant Journal 1997, 12 (6), 1465-1472.

2. $\quad$ Cha, S.; Zhang, H.; Ilarslan, H.; Wurtele, E.; Brachova, L.; Nikolau, B.; Yeung, E., Plant Journal 2008, 55 (2), 348-360.

3. Caprioli, R. M.; Farmer, T. B.; Gile, J., Anal. Chem. 1997, 69 (23), 4751-60.

4. $\quad$ Reyzer, M. L.; Hsieh, Y.; Ng, K.; Korfmacher, W. A.; Caprioli, R. M., J. Mass Spectrom. 2003, 38 (10), 1081-92.

5. $\quad$ Stoeckli, M.; Chaurand, P.; Hallahan, D.; Caprioli, R., Nature Medicine 2001, 7 (4), 493496.

6. Stoeckli, M.; Staab, D.; Staufenbiel, M.; Wiederhold, K. H.; Signor, L., Anal. Biochem. 2002, 311 (1), 33-9.

7. $\quad$ Rohner, T. C.; Staab, D.; Stoeckli, M., Mech. Ageing Dev. 2005, 126 (1), 177-85.

8. Cha, S.; Song, Z.; Nikolau, B. J.; Yeung, E. S., Anal. Chem. 2009, 81 (8), 2991-3000.

9. Burrell, M.; Earnshaw, C.; Clench, M., J. Exp. Bot. 2007, 58 (4), 757-63.

10. Ostrowski, S. G.; Szakal, C.; Kozole, J.; Roddy, T. P.; Xu, J.; Ewing, A. G.; Winograd, N., Anal. Chem. 2005, 77 (19), 6190-6.

11. Ifa, D. R.; Manicke, N. E.; Dill, A. L.; Cooks, R. G., Science. 2008, 321 (5890), 805.

12. Wiseman, J. M.; Ifa, D. R.; Venter, A.; Cooks, R. G., Nat Protoc 2008, 3 (3), 517-24.

13. Ute Bahr, A. D., Michael Karas, Franz Hillenkanp, Ulrich Giessmann., Anal. chem. 1992, 64 (22), 2866-2869.

14. Guo, Z.; Zhang, Q.; Zou, H.; Guo, B.; Ni, J., Anal. Chem. 2002, 74 (7), 1637-41.

15. Altelaar, A. F.; van Minnen, J.; Jimenez, C. R.; Heeren, R. M.; Piersma, S. R., Anal. Chem. 2005, 77 (3), 735-41.

16. Li, Y.; Shrestha, B.; Vertes, A., Anal. Chem. 2007, 79 (2), 523-32.

17. Kahr, M. S.; Wilkins, C. L., Journal of the American Society for Mass Spectrometry 1993, 4 (6), 453-460.

18. Dutta, T. K.; Harayama, S., Analytical chemistry 2001, 73 (5), 864-869.

19. Spengler, B.; Hubert, M., J. Am. Soc. Mass Spectrom. 2002, 13 (6), 735-48.

20. Chaurand, P.; Schriver, K. E.; Caprioli, R. M., J. Mass Spectrom. 2007, 42 (4), 476-89. 
21. Chaurand, P.; Angle, P. M.; Caprioli, R. M., Proc. 57th ASMS Conf. on Mass

Spectrometry and Allied Topics, Philadelphia, PA, May 31-June 4. 2009, Oral session MOAam10:10.

22. Hölscher, D.; Shroff, R.; Knop, K.; Gottschaldt, M.; Crecelius, A.; Schneider, B.; Heckel, D. G.; Schubert, U. S.; Svatos, A., Plant J 2009, 60 (5), 907-18.

23. Qiao, H.; Piyadasa, G.; Spicer, V.; Ens, W., Int. J. Mass Spectrom. 2009, 281 (1-2), 4151.

24. Shrestha, B.; Vertes, A., Anal. Chem. 2009, 81 (20), 8265-71.

25. Cha, S.; Yeung, E. S., Anal. Chem. 2007, 79 (6), 2373-85.

26. Landgraf, R. R.; Prieto Conaway, M. C.; Garrett, T. J.; Stacpoole, P. W.; Yost, R. A., Anal. Chem. 2009, 81 (20), 8488-95.

27. Chen, Y. F.; Allegood, J.; Liu, Y.; Wang, E.; Cachon-Gonzalez, B.; Cox, T. M.; Merrill, A. H.; Sullards, M. C., Anal. Chem. 2008, 80 (8), 2780-2788.

28. Strupat, K.; Kovtoun, V.; Bui, H.; Viner, R.; Stafford, G.; Horning, S., J. Am. Soc. Mass Spectrom. 2009, 20 (8), 1451-1463.

29. Alcantara, J. F.; Vadillo, J. M.; Laserna, J. J., Rapid Commun. Mass Spectrom. 2008, 22 (12), 1999-2005.

30. Y. Nakata, H. Y., Y. Honda, S. Ninomiya, T. Seki, T. Aoki, J. Matsuo, Nucl. Instrum. Meth. B. 2009, 267, 2144.

31. Samuels, L.; Kunst, L.; Jetter, R., Annu. Rev. Plant Biol. 2008, 59, 683-707.

32. Goodwin, S. M., Jenks, M.A., Plant Abiotic Stress 2005, Jenks, M.A., Hasegawa, P.M. eds., Oxford,U.K.

33. Pirman, D. A., Borum, P. R.; Yost, R. A., Proc. 57th ASMS Conf. on Mass Spectrometry and Allied Topics, Poster session ThPF165. 2009, May 31-June 4 (ThPF165.), Philadelphia.

34. Dinneny, J. R.; Weigel, D.; Yanofsky, M. F., Development. 2006, 133 (9), 1645-55.

35. Li, Y. H.; Beisson, F.; Ohlrogge, J.; Pollard, M., Plant Physiol. 2007, 144 (3), 1267-1277.

36. Andersson, M.; Groseclose, M. R.; Deutch, A. Y.; Caprioli, R. M., Nat. Methods. 2008, 5 (1), 101-8.

37. Crecelius, A. C.; Cornett, D. S.; Caprioli, R. M.; Williams, B.; Dawant, B. M.; Bodenheimer, B., J. Am. Soc. Mass Spectrom. 2005, 16 (7), 1093-9. 
38. Cha, S.; Zhang, H.; Ilarslan, H. I.; Wurtele, E. S.; Brachova, L.; Nikolau, B. J.; Yeung, E. S., Plant J. 2008, 55 (2), 348-60.

39. Luxembourg, S.; Mize, T.; McDonnell, L.; Heeren, R., Analytical Chemistry 2004, 76 (18), 5339-5344.

40. Vestal, M.; Hayden, K., Int. J. Mass Spectrom. 2007, 268 (2-3), 83-92. 


\section{Figure Captions}

Figure 1. Homogeneity of matrix applied: SEM images of bare stainless steel plates sprayed with colloidal silver using a commercial nebulizer (a) and oscillating capillary nebulizer without (b) or with (c) 4 times the volume of 2-propanol. Part (d) is a zoomed-in SEM image of one of the black specks shown in part (c).

Figure 2. MS imaging in high spatial resolution of single cell level demonstrated on an Arabidopsis flower. MS image of silver ion adduct of C29 alkane ( $\mathrm{m} / \mathrm{z} 515)$ on an Arabidopsis flower obtained with the spatial resolution of $12 \mu \mathrm{m}$ (12 $\mu \mathrm{m}$ for both laser spot size and raster size). Ion abundances are normalized against the silver dimer, $\left.\left[{ }^{107} \mathrm{Ag}+{ }^{109} \mathrm{Ag}\right]\right]^{+}(\mathrm{m} / \mathrm{z} 216)$. Zoomed-in images of the stigma and anthers demonstrate single cell level high spatial resolution. The letters indicate the positions of single pixels for the mass spectra shown in Figure 3.

Figure 3. Single pixel mass spectra representing each part of flower tissues for the positions indicated in Figure 2: (A) pollen grain, (B) side of carpel, (C) stamen filament, (D) tip of sepal, (E) middle of sepal, $(\mathrm{F})$ side of petal; * labeled peaks arise from contamination.

Figure 4. MS images of $\mathrm{C} 29$ ketone, $\left[\mathrm{C} 29\right.$ ketone $\left.+{ }^{107} \mathrm{Ag}\right]{ }^{+}(\mathrm{m} / \mathrm{z} 529)$, and C26 fatty acid, [C26 fatty acid $\left.+{ }^{107} \mathrm{Ag}\right]^{+}(\mathrm{m} / \mathrm{z} 503)$, normalized to the ion intensity of the silver dimer, $\left[{ }^{107} \mathrm{Ag}+{ }^{109}\right.$ $\mathrm{Ag}]^{+}(m / z 216)$. The maximum color scales are 0.5 for both images for the same color scheme shown in Figure 2.

Figure 5. Relative ion abundance profile constructed to demonstrate single cell level spatial resolution: (A) magnified image of a stamen at the upper part of the flower shown in Figure 2; (B) single pixel profiles for C29 alkane, C29 ketone, and C26 fatty acids as silver ion adducts $(\mathrm{m} / z$ 
515, 529, and 503, respectively) normalized to the silver dimer, $\left[{ }^{107} \mathrm{Ag}+{ }^{109} \mathrm{Ag}\right]^{+}(\mathrm{m} / \mathrm{z} 216)$, along a series of single pixels vertically at the $X$ position of $2100 \mu \mathrm{m}$ indicated by the arrow in the left figure for $Y$ positions of 2400-3000 $\mu \mathrm{m}$ from the base of the image in Figure 2. The ion signals fluctuate periodically, typically every two or three pixels, which matches with the longitudinal size of epidermal cell, $\sim 30 \mu \mathrm{m}$ on a stamen filament.

Figure 6. MS imaging of Arabidopsis root hairs: (left) optical image of Arabidopsis root and (right) MS image of silver ion adduct of $\mathrm{C}_{18}$ caffeate on an Arabidopsis root normalized with respect to the silver dimer, $\left[{ }^{107} \mathrm{Ag}+{ }^{109} \mathrm{Ag}\right]^{+}(\mathrm{m} / \mathrm{z} 216)$, acquired with $12 \mu \mathrm{m}$ spatial resolution.

Figure 7. Differentiation of isobaric ions with high-resolution mass spectrometry: (top) MS images of silver ion adduct of C29 alkane $(\mathrm{m} / \mathrm{z} 515.3728)$ and C28 aldehyde $(\mathrm{m} / \mathrm{z} 515.3376)$ obtained on an Arabidopsis stem with MALDI LTQ-Orbitrap with $50 \mu \mathrm{m}$ spatial resolution. Accurate mass imaging was constructed with mass tolerance of \pm 0.008 Da from their calculated masses. (Bottom) MALDI mass spectrum of an Arabidopsis stem shown for the $m / z$ range 514520 obtained with the MALDI LTQ-Orbitrap using colloidal silver as a matrix.

Figure 8. Depth resolved MS imaging of Arabidopsis stem. MS images of C24-C30 fatty acids and kaempferol on an Arabidopsis stem obtained before (top row) and after (bottom row) surface sputtering with 480 laser shots at maximum laser power of $80 \mu \mathrm{J} /$ pulse. Colloidal graphite was used as a matrix and MALDI LTQ-Orbitrap was used for data acquisition with $50 \mu \mathrm{m}$ spatial resolution. The ion signals are normalized with respect to total ion current (TIC). 

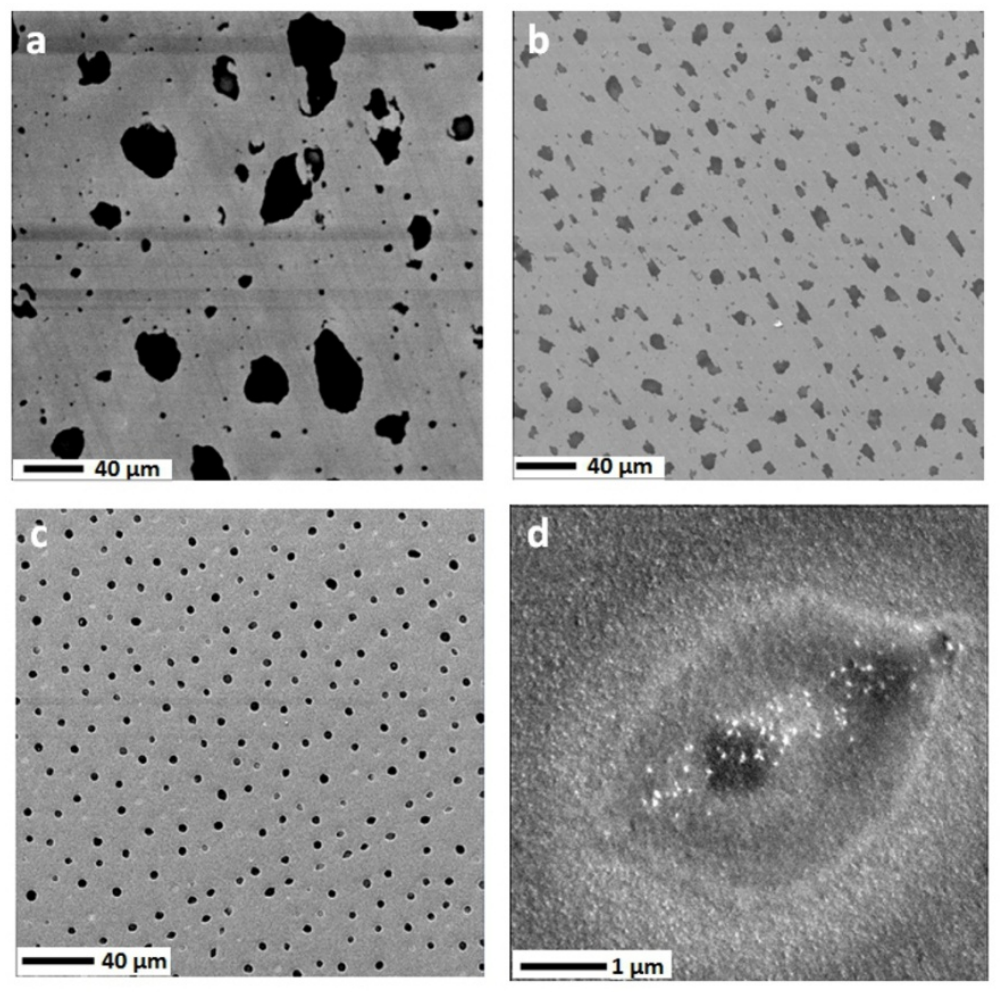

Figure 1. 


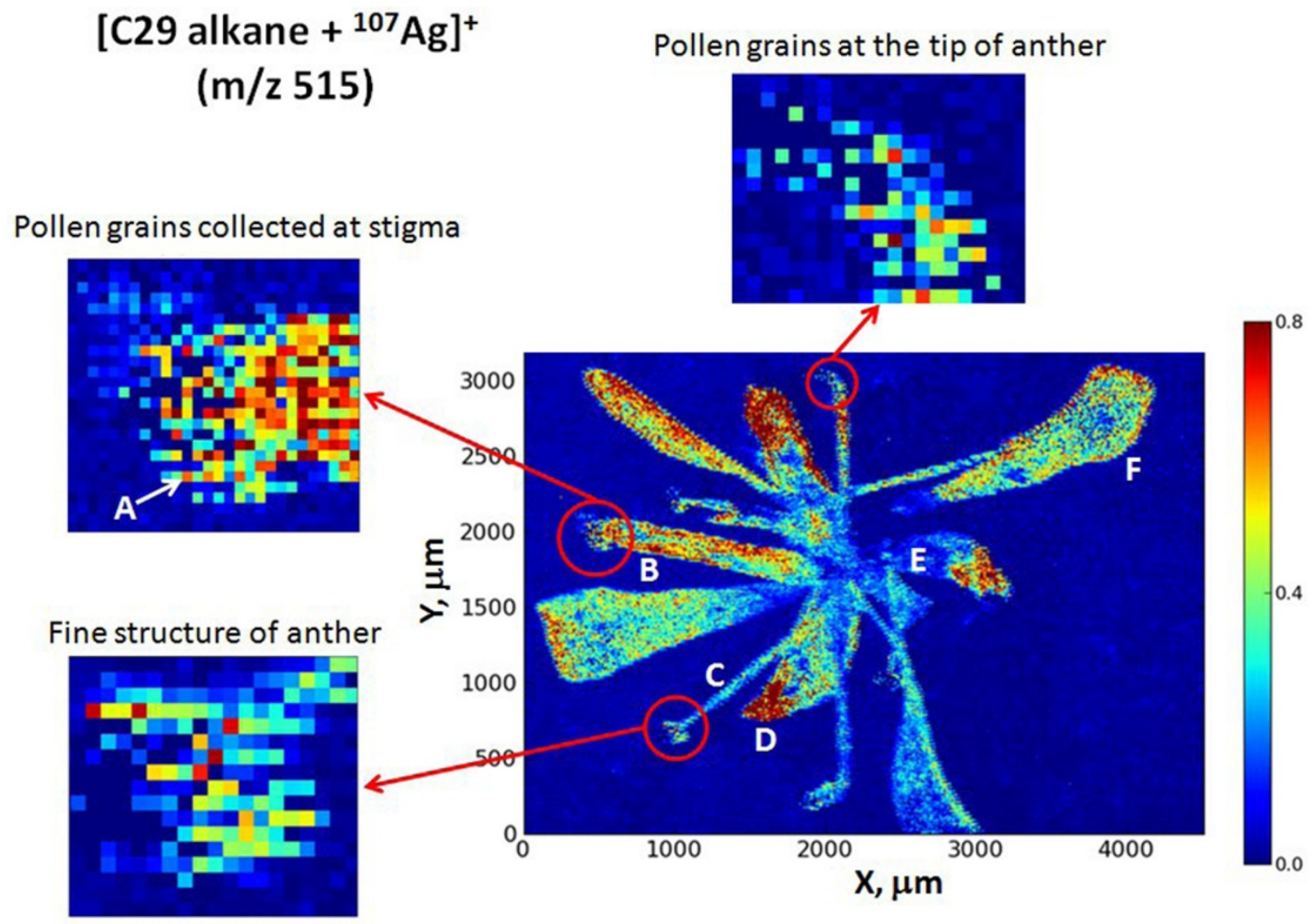

Figure 2. 

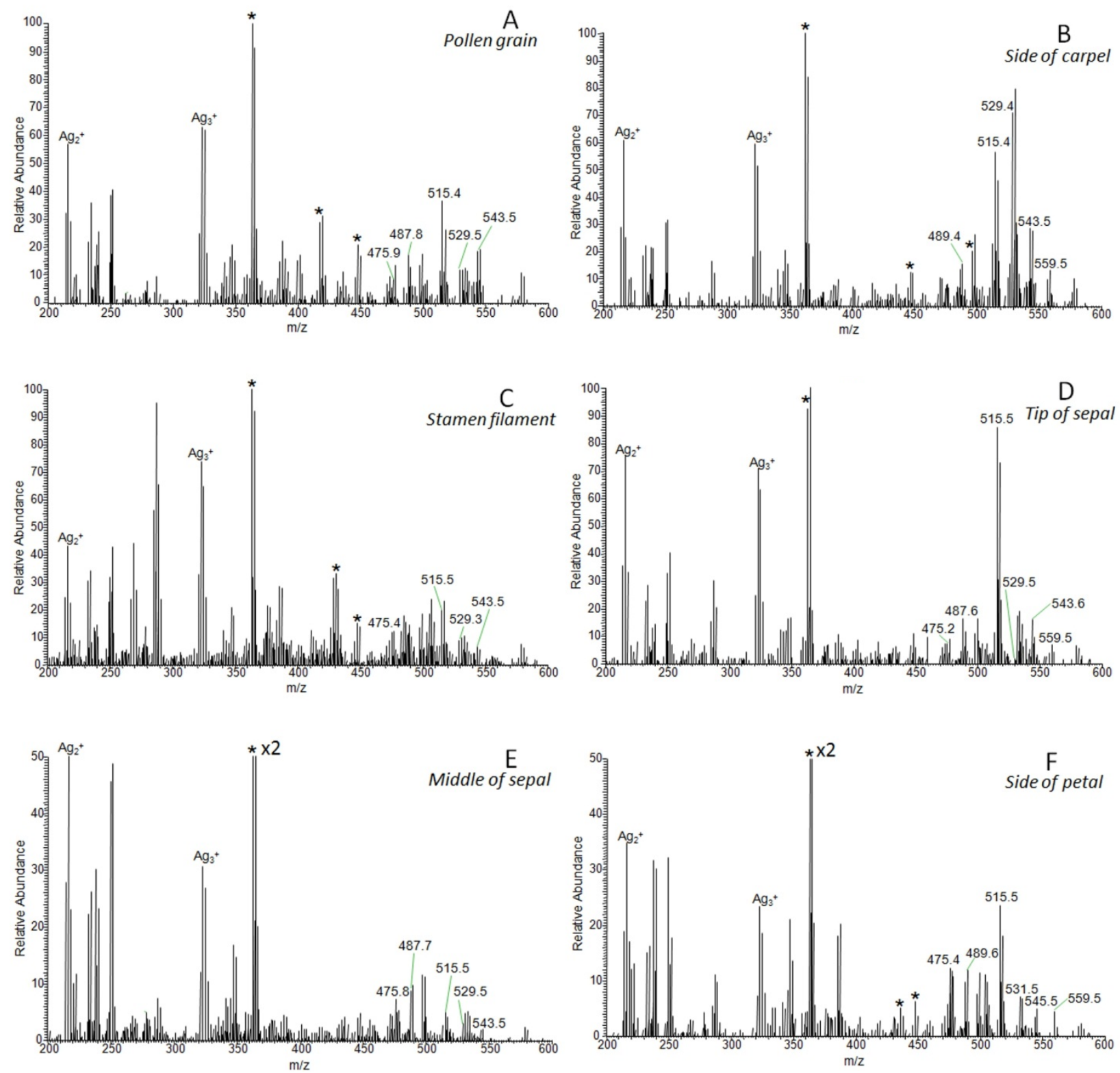

Figure 3. 

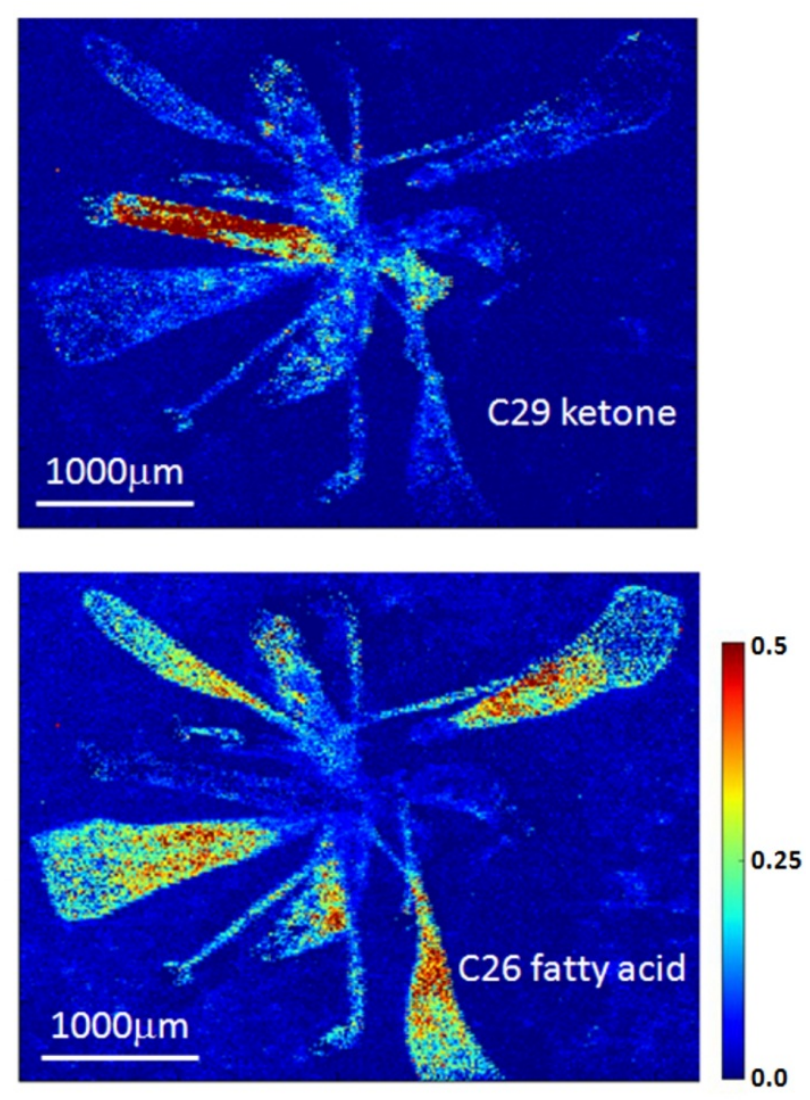

Figure 4. 

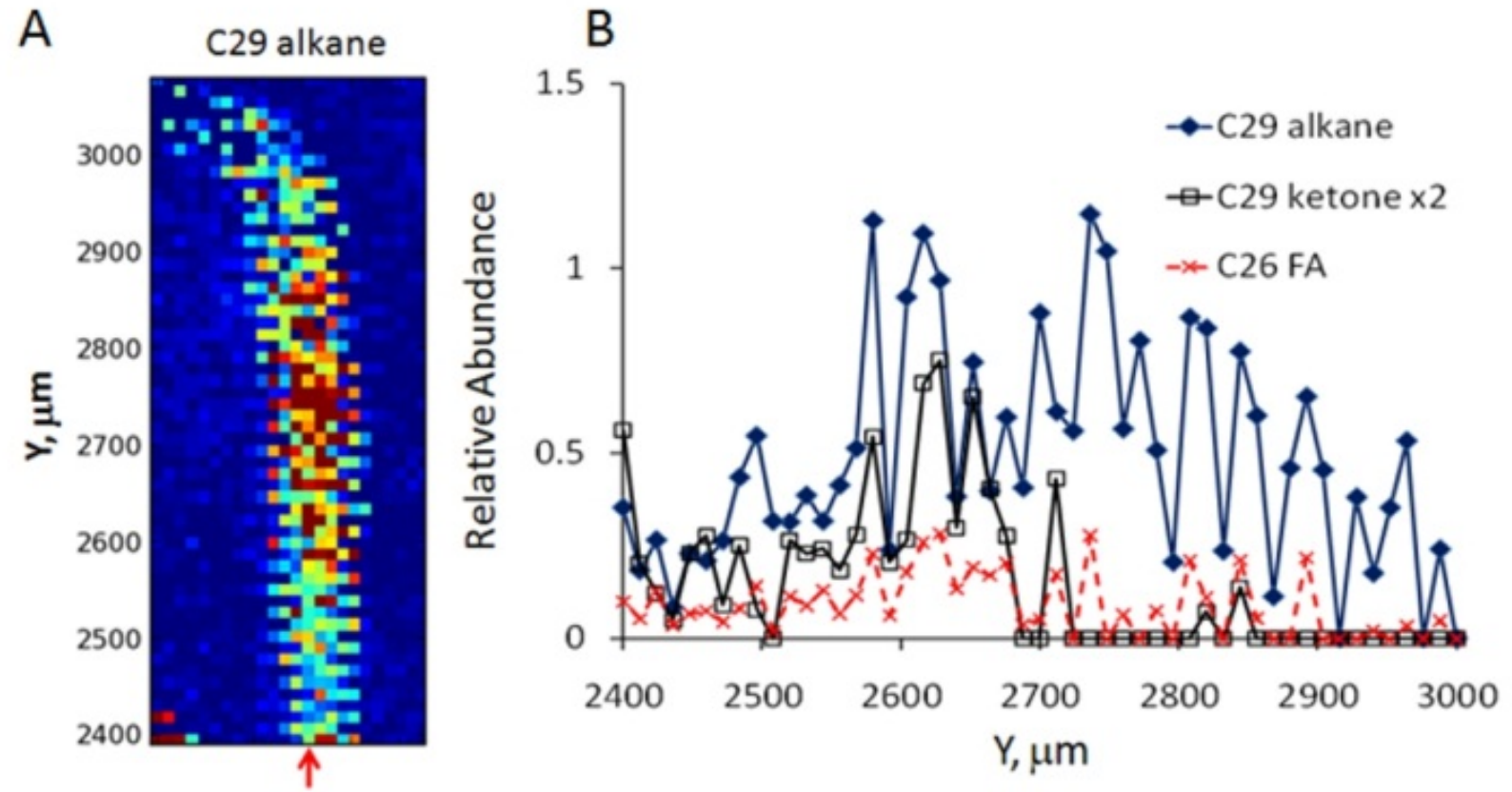

Figure 5. 


\section{[C18 Caffeate $\left.{ }^{107} \mathrm{Ag}\right]^{+}$ (m/z 539)}

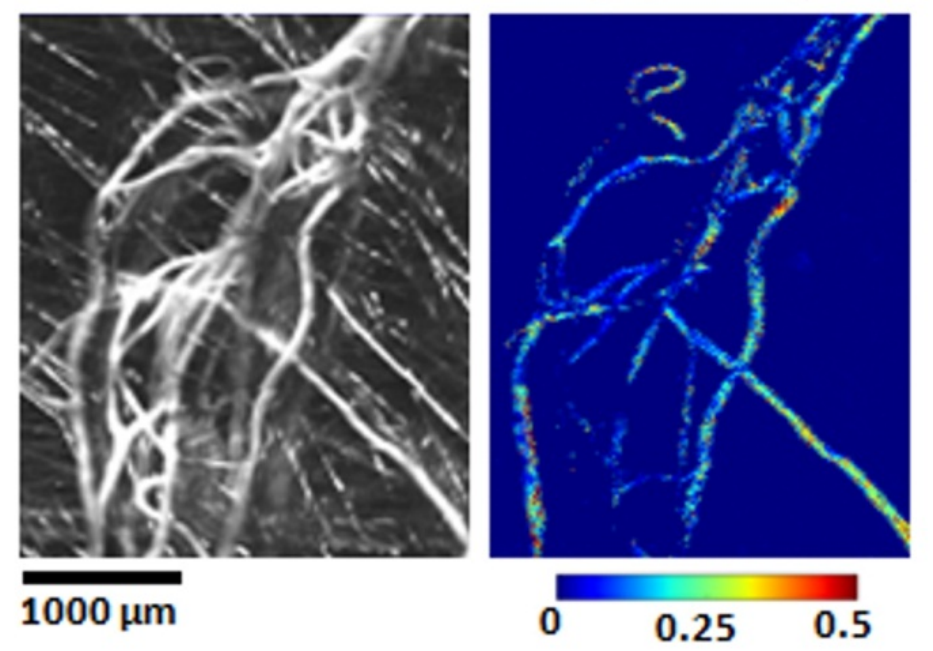

Figure 6. 


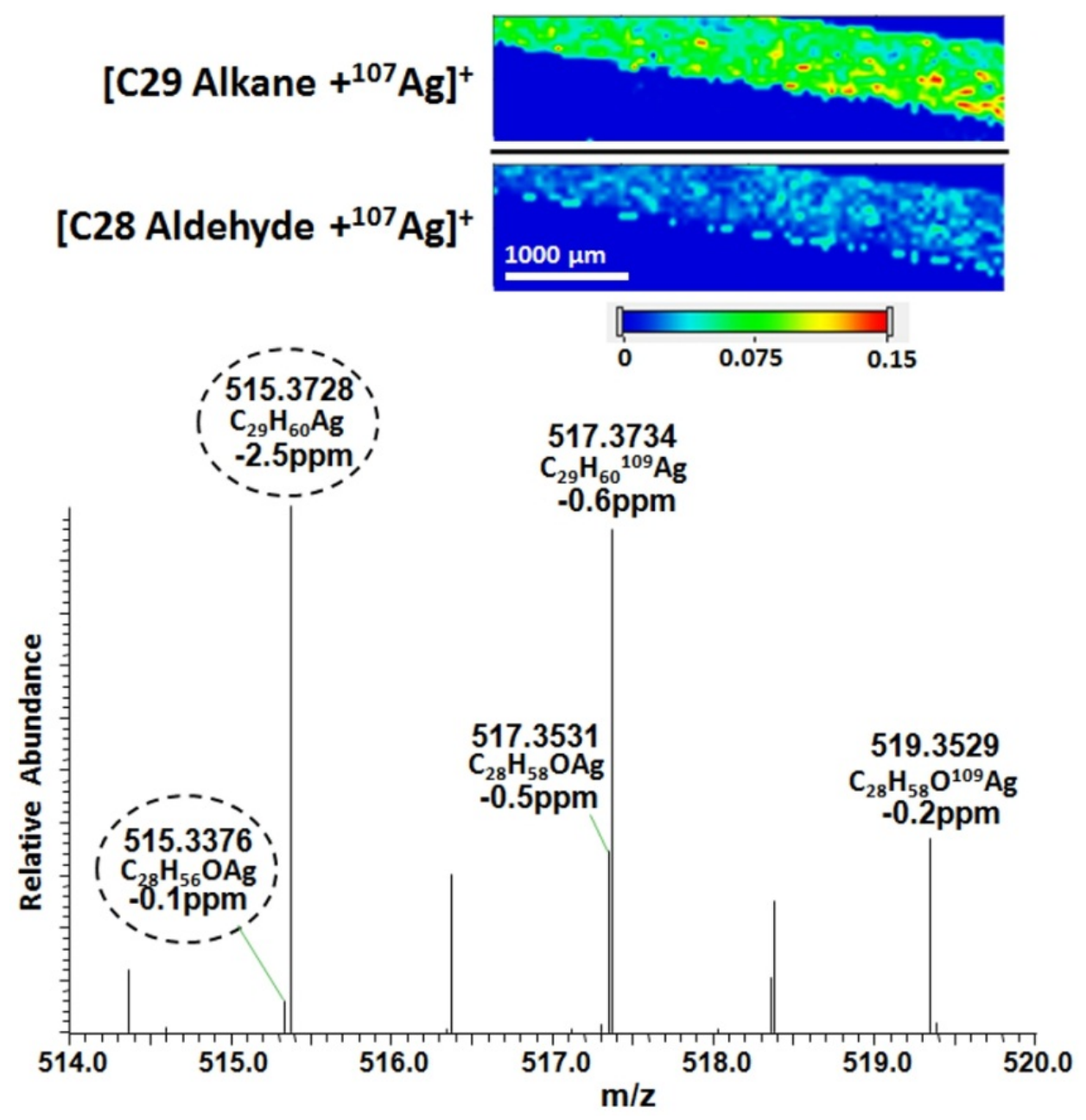

Figure 7. 


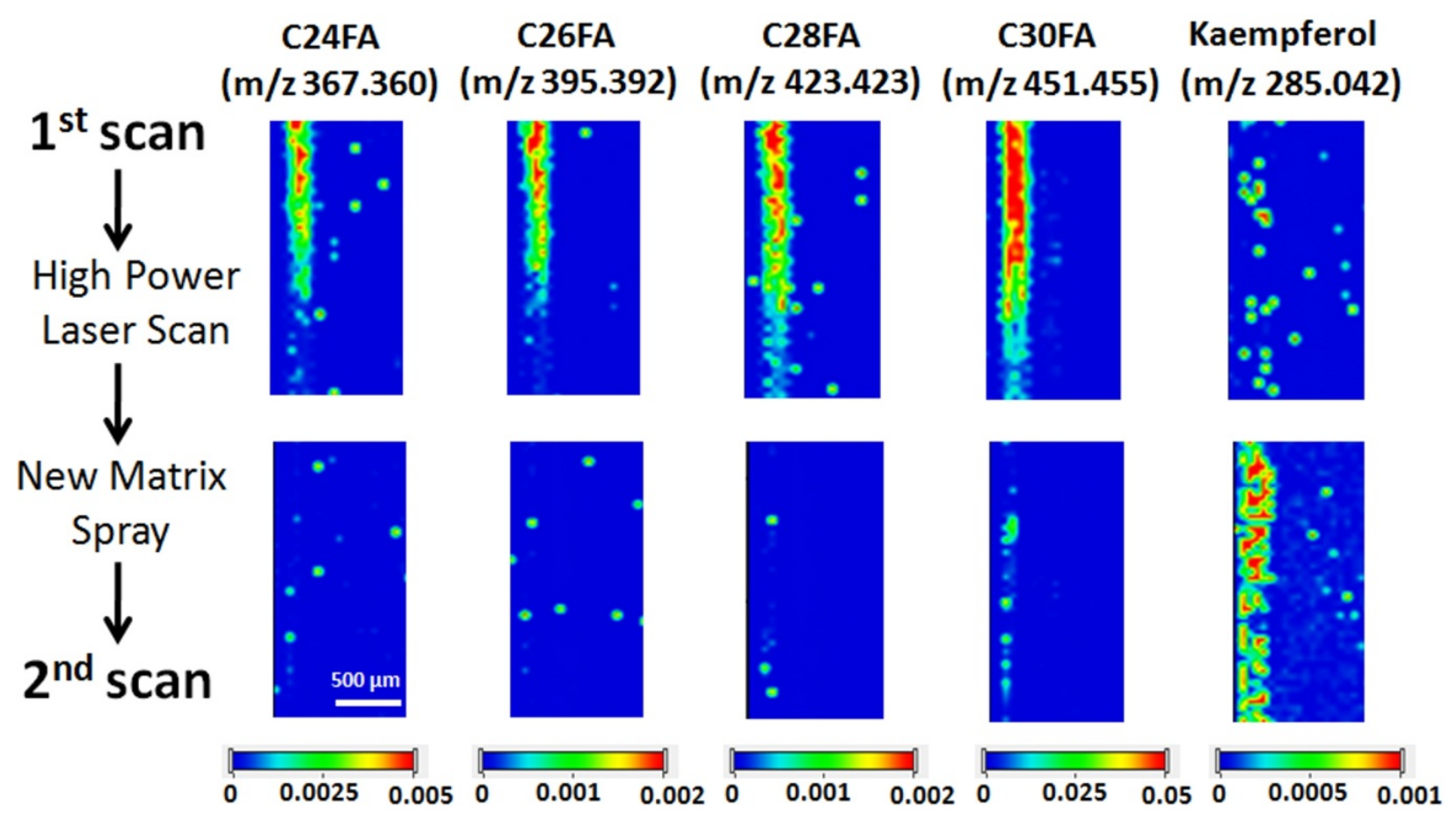

Figure 8. 


\title{
CHAPTER 3. MASS SPECTROMETRIC IMAGING ON VARIOUS ORGANS OF ARABIDOPSIS MUTANT WITH HIGH SPATIAL AND HIGH MASS RESOLUTION FOR REVELATION OF PLANT METABOLITES DISTRIBUTION ALTERED BY GENETIC MUTATION
}

\author{
Manuscript in preparation for submission
}

Ji Hyun Jun, Zhenjiu Liu, Zhihong Song, Basil J. Nikolau, Edward S. Yeung, and Young Jin Lee

\begin{abstract}
The high spatial and high mass resolution technology has been developed for plant metabolites mass spectrometric (MS) imaging. The distribution of plant metabolites is asymmetric throughout the cells and tissues and MS imaging is expected to reveal the relevant cellular and molecular biology in unprecedented depth. We applied our technology to cer 1 mutant of the model system Arabidopsis thaliana to demonstrate its usefulness and reveal associated metabolite changes compared to those of wild-type. High spatial resolution of $\sim 12-50$ $\mu \mathrm{m}$ was utilized to visualize minute features of chemical distributions in flowers and roots along with other plant organs such as stems and leaves, and this is the highest spatial resolution ever obtained and/or utilized for plant metabolite imaging. High resolution mass spectrometry using LTQ-Orbitrap with mass accuracy of 5 ppm or less was adopted to differentiate isobaric metabolites in MALDI imaging. With this new instrumentation, we could successfully
\end{abstract}


differentiate important classes of isobaric metabolites, i.e. C29 alkane vs. C28 aldehyde, and directly confirm chemical identities of unknown metabolites through chemical composition analysis. Based on this, separate MS images of differentiated isobaric metabolites could be constructed on the two genotypes and this allows tracking of abundance changes for those compounds along with the genetic mutation, which is not achievable with low mass resolution mass spectrometry . Systematic investigations of various plant organs supported previous hypothesis and also revealed new biology. Specifically, the decreased abundance level of alkanes, C29 ketones, C26 fatty acids, and C28 fatty acids and the increment in aldehydes, C30 fatty acids abundance level are consistently observed on various organs of (flowers, leaves, stems) of cer 1 mutant by MS imaging. Above all, our results support suggested hypothesis of molecular function of $C E R 1$ gene as aldehyde decarbonylase, especially by displaying hyper accumulation of aldehydes and C30 fatty acid and decrease in abundance of alkanes and ketones in several plant organs of cer 1 mutant.

\section{Introduction}

MS imaging unveils the spatial distribution of various analytes and their abundance level on the targeted biological tissue. The combination of MALDI (Matrix-assisted laser desorption/ionization) with high dynamic range and mass spectrometry (MS) capable of high chemical specificity and sensitivity can be used not only for profiling purpose at the discrete points of the sample but also for imaging across the entire sample in a consecutive manner. Although MALDI MS imaging was originally applied to the analysis of peptides and proteins ${ }^{1}$,

its application was also extended to drugs and their metabolites ${ }^{2}$, pathological studies ${ }^{3-5}$, and 
plant metabolites ${ }^{6-9}$ along with technical improvements in instrumentation and sample preparation.

The two key factors determining high spatial resolution in MALDI MS imaging are laser spot size and homogeneity of matrix application. There have been many efforts to reduce the laser spot diameter such as using pinhole and focusing lens ${ }^{1}$, or the multiple focusing lenses ${ }^{10}$. Recently, Caprioli's group used coaxial laser illumination to achieve high spatial resolution in the MS imaging ${ }^{11}$ and the spatial resolution of $4.8 \mu \mathrm{m}$ was obtained in the MS imaging on the mouse brain ${ }^{12}$. Achievement of the MS imaging in single cell level $(\sim 10 \mu \mathrm{m})$ using the smartbeam technology in the Ultraflex III time-of-flight (TOF) mass spectrometer has been also reported, in which the distribution of UV-absorbing secondary metabolites in plant tissues were visualized with no matrix ${ }^{8}$. High resolution MS imaging has been previously performed on single crystals formed by standard analyte samples mixed with matrix on the target plate ${ }^{13}$ using an optical fiber, a convenient way to reduce the laser beam size. In this current study, we utilized an optical fiber with a 25-100 $\mu \mathrm{m}$ core diameter to investigate the altered distribution of plant metabolites on various organs of $c e r 1$ mutant upon $C E R 1$ gene mutation in parallel to the wildtype (Ler). MS imaging of Arabidopsis flowers, leaves, stems, and roots was successfully accomplished with a spatial resolution of 12 or $50 \mu \mathrm{m}$.

Likewise, more homogenous application of matrix is a very important matter of which insufficiency can cause the analytes migration, spatial information of artifact or degradation in the spatial resolution. For this reason, many different approaches have been tried to deposit the matrix more uniformly on the sample, including matrix application through electrospray ${ }^{14,15}$ or an acoustic droplet ejector ${ }^{16}$, immersing the sample in matrix solution ${ }^{17}$, and vapor-phase deposition of matrix through sublimation ${ }^{18}$. We adopted the use of oscillating capillary nebulizer 
developed by Sullards and co-workers ${ }^{19}$ and applied the matrix with a modified commercial airbrush. Colloidal silver previously has been used as an additive for MS imaging of lipid metabolites on plant surfaces in our group ${ }^{6}$. This novel matrix makes it easier to distinguish the peaks attributed to metabolites from those from the background by forming the intact silver $\left({ }^{107} \mathrm{Ag},{ }^{109} \mathrm{Ag}\right)$ adduct ions with different classes of cuticular wax compounds. Moreover, the metabolite peaks are not obscured by those from matrix, which has been a significant problem for other organic matrixes in the low $\mathrm{m} / \mathrm{z}$ ranges. The homogeneity of colloidal silver on the hydrophobic plant surface also makes it more suitable as a matrix in plant metabolites imaging.

Among various applications of MS imaging mentioned earlier, MS imaging can be effectively used for the elucidation of identity and spatial distribution of metabolites within the plant tissues. Even though MS imaging has been rigorously utilized for biomedical studies ${ }^{2-5}$ its application to plant metabolites is still infancy. For example, the distribution of amino acids, sugars, and phosphorylated metabolites in wheat seeds has been studied by MS imaging in the preceding study ${ }^{7}$. Although the number of publications related to MS imaging on plant materials is increasing recently, reports of the utilization of this technique for biomedical studies are still more common.

Cuticular wax is the plant metabolite placed on the outer surface of the plant $(0.1-1 \mu \mathrm{m})^{20}$, so it protects the plants from the external stresses such as UV irradiation, pathogen, insect, water loss and frost ${ }^{21}$. Previously, many research groups have investigated the role of various $C E R$ genes in cuticular wax formation on the different organs of Arabidopsis thaliana ${ }^{22-33}$. The functional characterization of specifically mutated genes helps to understand details of cuticular wax biosynthesis. This understanding may provide the possibilities to regulate wax formation on the plant, which in turn also greatly affects the quality of the crops. CER 1 gene is suggested to 
play a role in the conversion of aldehydes to alkanes in the Arabidopsis wax biosynthetic pathway ${ }^{23-26,29,30,34}$, none of previous research has shown the spatial distribution of CER 1 gene products.

In this paper, the high spatial/mass resolution of MS imaging was effectively applied to reveal the epicuticular wax distribution altered by $C E R 1$ genetic mutation and address functional role of CER 1 gene in the aspects of spatial distribution of its products. The reduction of laser beam diameter allowed us to achieve higher spatial resolution in MS imaging. In addition, Orbitrap mass spectrometer having high mass resolving power with high mass accuracy was adopted to overcome the previous limitation ${ }^{6}$ arising from the overlapped isobaric metabolites (such as $\mathrm{C} 29$ alkane, $\mathrm{C} 28$ aldehyde; $m / z$ 515). Although other mass analyzers with high mass resolution capability such as FTICR (Fourier transform ion cyclotron resonance) ${ }^{35-37}$ or quadrupole timeof-flight (QTOF) MS ${ }^{38}$ have been also utilized with MALDI source for imaging purpose, these previous studies had either lower throughput or spatial resolution. The resolved peaks were differentiated into different species and their identities were directly confirmed through chemical composition analysis even without MS/MS data. The acquisition of individual MS images for isobaric metabolites is one of the most prominent advances in our work. By imaging the epicuticular waxes systematically on the various organs of eceriferum mutant (cer1), the associated alterations in their spatial distribution with genetic mutation of $C E R 1$ were investigated to characterize the functional role of $C E R 1$ gene in cuticular wax biosynthesis. The knowledge obtained from this study will be useful indeed to study how the CER 1 gene mutation is spatially expressed on the different organs and the detailed function of $C E R 1$ gene in the wax biosynthetic pathway of Arabidopsis. 


\section{Experimental Section}

Chemicals. Collloidal silver (20 ppm) used as a matrix was purchased from Purest Colloids, Inc. (Westampton, NJ). Colloidal graphite aerosol spray was obtained from Alfa Aesar (Ward Hill, MA, www.alfa.com). 2-propanol (HPLC grade) was purchased from Fisher Scientific (Fairlawn, NJ). N,O-Bis (trimethylsilyl)triflyoroacetamide with trimethylchlorosilane (BSTFA/TMCS), Murashige and Skoog basal salt mixture, and standard compound docosane were purchased from Sigma-Aldrich (St. Louis, MO).

Plant Growth Conditions. Arabidopsis thaliana ecotype Landsberg erecta (Ler-0) and eceriferum mutant (cer1, genetic stock CS31) were obtained from the Arabidopsis Biological Resource center. Seeds were sown on MS (Murashige and Skoog basal salt mixture) media in petri dishes. The dishes were placed in the growth room for 10 days after keeping at $4^{\circ} \mathrm{C}$ for 4 days. On the 15 th day the plants were transferred in soil for continuous growth. Flowers were collected on the 45th day, leaves were collected on the 14th day, and stems were collected on the 28th day for the data acquisition with both low resolution and high resolution MS. The flower sample used for MS imaging obtained with $12 \mu \mathrm{m}$ spatial resolution was collected on the 42nd day. For the samples used for flavonoid imaging, the flowers were collected on the 43th day. The

growth room was set at $24^{\circ} \mathrm{C}$ with an illumination at $85 \mu \mathrm{E} \mathrm{m} \mathrm{m}^{-1}$ and ambient relative humidity. For the root samples, seeds were sterilized and cultured in $500 \mathrm{~mL}$ flasks containing $250 \mathrm{~mL} \mathrm{MS}$ medium for 14 days at room temperature. The shaker speed was $100 \mathrm{rpm}$. The roots were 46 days old when they were isolated from the media.

Sample Preparation for LDI MS Imaging. After each plant organ was collected from the plant batch, they were pretreated before the mass spectrometry imaging analysis. The samples were attached onto the stainless steel target plate using double-sided conductive tape $(3 \mathrm{M}$, St. 
Paul, MN). Air pressure from the $\mathrm{N}_{2}$ gas cylinder was applied to help the sample attach firmly to the sample plate without any physical damages on the plant tissue. Also, the targeted area for imaging purpose was not touched with the forceps. The attached samples were dried under the moderate vacuum ( $\sim 50$ Torr) for 30-60 min to minimize metabolite turnover.

A commercial airbrush (Aztek A470 from Testor, Rockford, IL) is modified to an oscillating spray device ${ }^{19}$ by replacing the spray tip with a capillary with $100 \mu \mathrm{m}$ I.D. for homogeneous matrix application. Matrixes were transferred to the modified air brush through syringe pump (Fisher Scientific, Pittsburgh, PA) and sprayed onto the plant tissue by nebulizing with $\mathrm{N}_{2}$ gas. For the colloidal silver, $0.15 \mathrm{~mL}$ of solution was sprayed at $50 \mu \mathrm{L} \mathrm{min}{ }^{-1}$ of flow rate. Unless the spraying is separately performed with appropriate time gap, the droplets in proximity are likely to form aggregation which consequently leads to the analytes migration. For this reason, the time interval for air-drying was ensured between each application with a certain volume of matrix solution. For the colloidal graphite, the colloidal graphite from aerosol spray was first mixed with 2-propanol at the volumetric ratio of 1:8. The $0.30 \mathrm{~mL}$ of colloidal graphite dissolved in the 2-propanol was sprayed on the sample at $50 \mu \mathrm{L} \mathrm{min}^{-1}$ of flow rate. The distance between the tip of capillary equipped within the airbrush and the sample plate was $9 \mathrm{~cm}$ and the pressure of $\mathrm{N}_{2}$ gas was set to $40 \mathrm{psi}$ for both matrixes.

Sample Preparation for Gas Chromatography/Mass Spectrometry. Collected flowers, leaves or stems were weighed. An aliquot of internal standard (docosane) was added to the surface of the plant material. The cuticular waxes were extracted by completely immersing plant materials in chloroform for 60 seconds, and then dried under nitrogen gas. Samples were derivatized using BSTFA/TCMS $\left(65^{\circ} \mathrm{C}, 30\right.$ minutes) for GC/MS analysis. Three replicas of flowers, leaves, and stems were prepared. 
Mass Spectrometry Imaging. Mass spectrometric images were acquired with either a MALDI linear ion trap (Thermo Finnigan, vMALDI LTQ; San Jose, CA) or a high resolution MALDI mass spectrometer (Thermo Finnigan, LTQ-Orbitrap Discovery).

In vMALDI LTQ, the $\mathrm{N}_{2}$ laser system from Spectral Physics was used with maximum energy of $280 \mu \mathrm{J} /$ pulse, maximum frequency of $20 \mathrm{~Hz}$. The laser beam size could be reduced by changing optical fiber to the one with smaller core diameter (Oz Optics Ltd., Ottawa, ON, Canada). Optical fibers with core diameter of 25-100 $\mu \mathrm{m}$ were used and the typical laser beam size was half the optical fiber size after re-focusing with the lens. The MALDI source pressure was kept at $170 \mathrm{mTorr}$ in this mass spectrometer.

MALDI LTQ-Orbitrap also uses the $\mathrm{N}_{2}$ laser from LTB (model MNL 100; Lasertechnik Berlin GmbH, Berlin, Germany) with the maximum energy of $80 \mu \mathrm{J} /$ pulse, maximum repetition rate of $60 \mathrm{~Hz}$. Unlike the vMALDI LTQ, MALDI LTQ-Orbitrap has laser system directly attached to the mass spectrometer without guidance by optical fiber. The laser beam size was reduced to $25 \mu \mathrm{m}$ by placing a black-painted stainless steel plate with a pinhole aperture with a diameter of $3 \mathrm{~mm}$ in the laser beam path. The MALDI source pressure was maintained at 80 mTorr in this instrument.

Data Acquisition and Processing for Mass Spectrometric Imaging. For the plant tissues coated with colloidal silver, the data were acquired with a fixed number of laser shots without turning AGC (automatic gain control; varies the number of laser shots to gain the ion amounts for target value of analytical scan) on. The optimized number of laser shots and laser power were determined based on the test on the small area of sample surface with AGC-on.

For the colloidal graphite coated flower tissues, the data were collected with AGC-on since the colloidal graphite coating on the sample is relatively thicker than the one from colloidal 
silver. Thus, thick coating of colloidal graphite makes it hard to determine the optimized number of laser shots manually, even if this is tested on a small area of the plant tissue. The samples were scanned with a raster size of $12-50 \mu \mathrm{m}$ for the chosen $\mathrm{x}-\mathrm{y}$ areas with the mass spectral $\mathrm{m} / \mathrm{z}$ range of 200 to 800 .

All mass spectrometric images were generated with a tissue imaging visualization software (ImageQuest 1.0.1; Thermo Finnigan). The signal intensity of metabolite of interest was normalized by the silver dimer peak intensity $\left(\mathrm{m} / \mathrm{z} 216 ;\left[{ }^{107} \mathrm{Ag}+{ }^{109} \mathrm{Ag}\right]^{+}\right)$on each data point with the mass window of \pm 0.5 and \pm 0.008 Da for LTQ and LTQ-Orbitrap data, respectively. The mass spectrometric images of each metabolite were scaled with the same minimum, maximum value for both genotypes. For the cases where data sets could not be properly displayed as MS images with ImageQuest, ion signals were extracted for each pixel using a custom program provided by Thermo (vMALDIDataExtract) and MS images were constructed using a homemade program written in Python (version 2.5; http://www.python.org/) with a Matplot library (http://matplotlibsourceforge.net/).

Gas Chromatography/Mass Spectrometry. GC-MS analysis was performed with an Agilent $6890 \mathrm{GC}$ and 5973N mass spectrometer (Agilent Technologies). The HP-5ms column (30 $\mathrm{m} \times 0.25 \mathrm{~mm}$ i.d. coated with a $0.25 \mu \mathrm{m}$ film, Agilent Technologies) was used, and temperature gradient was programmed from 80 to $320{ }^{\circ} \mathrm{C}$ at $5^{\circ} \mathrm{C} / \mathrm{min}$ with $\mathrm{He}$ flow rate at 2.0 $\mathrm{mL} / \mathrm{min}$. Operating parameters were set to $70 \mathrm{eV}$ (electron ionization) of ionization voltage and $280^{\circ} \mathrm{C}$ of interface temperature. The GC/MS data files were deconvoluted by NIST AMDIS software, and searched against home-built mass spectral library and NIST05 spectral library. 


\section{Results and Discussion}

The Arabidopsis wax biosynthetic pathway (Schematic Diagram) needs to be briefly explained for better understanding of experimental results in connection with biological aspects. According to the simplified pathway ${ }^{39}, \mathrm{C} 16$ and C18 FA are formed in the beginning of biosynthetic pathway. VLCFAs (very long chain fatty acids; C20-C34 FA) are formed through fatty acid elongation of these precursor compounds. Then, the VLCFAs are modified into the components of cuticular waxes such as aldehydes, alkanes, ketones, and alcohols. For the formation of these wax compounds, VLCFAs undergo two separate pathways designated as primary alcohol pathway and alkane pathway. In the former, elongated FAs are successively converted to primary alcohols and wax esters. In the latter, aldehydes are first formed, and undergo decarbonylation and oxidations to produce alkanes, secondary alcohols and ketones in series.

MS Imaging of Epicuticular Waxes on the Arabidopsis Whole Flowers. We have recently achieved high spatial resolution of $12 \mu \mathrm{m}^{9}$ to $50 \mu \mathrm{m}$ by adopting optical fiber of smaller core diameter than that previously utilized for MS imaging of epicuticular waxes on Arabidopsis with a spatial resolution of $100 \mu \mathrm{m}^{6}$. Figure 1 shows the distinct differences in the quality of the MS images obtainable at different spatial resolution. As seen in the comparison of three MS images at $100 \mu \mathrm{m}$ (Figure 1; left), $50 \mu \mathrm{m}$ (middle), $12 \mu \mathrm{m}$ (right) spatial resolution, the MS image with higher spatial resolution allows the more detailed information in terms of spatial distribution of analytes of interest. The spatial distribution of the two different surface metabolites (C29 alkane, C29 ketone) is shown in Figure S-2. The different localization of each metabolite is visualized by MS imaging at high spatial resolution. Figure S-2 (in Appendix 2) also shows the importance of achieving high spatial resolution in MS imaging to better understand the relevant cellular and 
molecular biology in unprecedented depth. In the present work, we applied this high spatial resolution to plant metabolite imaging. For most studies, the spatial resolution of $50 \mu \mathrm{m}$ was mostly used for MS imaging of surface metabolites on various organs to compromise a long data acquisition time considering the number of organs targeted for this study. Because the fine root hairs necessitates higher spatial resolution compared to other organs (flowers, leaves, or stems), the use of the spatial resolution of $12 \mu \mathrm{m}$ was essential for root imaging.

The first organ examined was flower because it is composed of various suborgans and can best demonstrate our capability of improved spatial resolution. The utility of colloidal silver as a matrix previously turned out to be effective for the detection of cuticular waxes on the plant tissue ${ }^{6}$. The formation of silver adduct ions $\left(\left[\text { cuticular wax }+{ }^{107} \mathrm{Ag}\right]^{+},\left[\text {cuticular wax }+{ }^{109} \mathrm{Ag}\right]^{+}\right)$ having a difference in mass-to-charge ratio $(\mathrm{m} / \mathrm{z})$ by $2 \mathrm{Da}$ due to silver isotopes made it easier to distinguish the peaks attributed to the metabolites from those from the background. For instance, if any metabolite candidate was not detected as doublet peaks attributed to the silver isotopes $\left({ }^{107} \mathrm{Ag},{ }^{109} \mathrm{Ag}\right)$, those candidates were not considered real surface metabolites.

The distribution of several representative cuticular waxes on the Arabidopsis flower was visualized by MS imaging with a spatial resolution of $50 \mu \mathrm{m}$ in Figure 2. The data were collected with MALDI linear ion trap (vMALDI LTQ) where the optical fiber is used to guide the laser beam to the sample. The optical fiber brings into the laser beam in size half of its core diameter. Compared to the previous work where the data were obtained at spatial resolution of $50 \mu \mathrm{m}$ by oversampling method (with $100 \mu \mathrm{m}$ of laser beam size) ${ }^{6}$, the spatial distribution of wax can be now visualized in more details at higher resolution by utilizing the optical fiber with the smaller core diameter to reduce the laser spot size to $12-50 \mu \mathrm{m}$. In the previous work, even though oversampling method allowed the high spatial resolution without the actual reduction of laser 
beam size by modifying the laser optics, the spatial resolution compromises the sensitivity for detection of analytes ${ }^{40}$. Thus, the actual decrease in laser spot diameter achieved by fiber optics with smaller size makes it possible to acquire the high spatial resolution for MS images without any sacrifice of signal level for analytes. For example, the distribution of $\mathrm{C} 29$ alkane is now clearly shown on the stamen in the present work (Figure 1). On the contrary, due to the diminutive nature of stamen among the flower suborgans, the spatial distribution of any wax compound on the stamen was hardly visualized at $50 \mu \mathrm{m}$ resolution by oversampling technique ${ }^{6}$.

For the wild-type (Ler), C29 alkane ( $\mathrm{m} / \mathrm{z} 515)$, the most abundant compound according to inparalleled GC-MS analysis (Table 1), is distributed on the overall suborgans of the whole flower at varied intensities. Among the various flower suborgans, it is observed with the highest abundance on the carpel. C29 alkane is also found on the petal, sepal and stamen at the lower intensities than that on the carpel. The value of MS imaging can be highlighted with the information about spatial localization of wax because this cannot be obtained with conventional GC-MS method. The color variation on the MS images over the flower can give an indication of the abundance of wax compound. However, it is noteworthy that ionization efficiency of the wax compounds may vary depending on the suborgans because each suborgan has a different surface characteristic. In LDI-MS with colloidal silver as a matrix, the abundance of epicuticular waxes is presumably determined by desorption efficiency of the analytes from the plant tissue surface of different characteristics as well as the efficiency of energy transfer from the matrix to anlaytes in the process of silver adduct ion formation in gas-phase. Based on the fact that the maximum plot values for MS images of, for example, C29 alkane and C29 ketone are close each other despite the excessive abundance of C29 alkane compared to the latter, it can be deduced that the energy transfer efficiency from the matrix to desorbed compound is much lower for C29 alkane 
than for $\mathrm{C} 29$ ketone. In other words, the matrix effect is minimal on determination of abundance level of wax compounds, but it is the desorption efficiency that plays more role in it. This also implies the potential for quantification of ions detected using internal standard in colloidal silver matrix with a prior knowledge of different desorption efficiencies of analytes on different suborgans.

The distribution of $\mathrm{C} 29$ ketone $(\mathrm{m} / \mathrm{z}$ 529) on the wild-type is somewhat similar to that of C29 alkane in that it is detected on the carpel at the highest level, then sepal and stamen. While the C29 alkane is detected from the most part of petal, the less uniform distribution on the petal was observed for the $\mathrm{C} 29$ ketone.

When the abundances of these two wax compounds are compared between the wild-type and the mutant, it is clear that the abundance levels of both compounds are higher in the wild-type than in the mutant. The presence of the C29 alkane is almost negligible on the mutant (cer 1) except for the specific parts such as carpel and tip of sepal and the C29 ketone is shown only on the carpel in weak signals. According to semi-quantified relative abundances (\%) from the MS images (Table 1), the abundance levels of the C29 alkane, C29 ketone on the carpel of wild-type are more than ten times higher than those on the mutant. Even though the differences of the wax amount on the petal from each genotype were less than on the carpel, two compounds also exist at higher abundance level on the petal of the wild-type than the mutant. The wax abundances of C29 alkane and C29 ketone revealed by GC-MS method are also in accordance with the trends observed on the MS images. These observations by both methods are consistent with the expectation based on the previous reports about the function of $C E R 1$ gene in wax biosynthetic pathway ${ }^{23-26,29-30,34,41-42}$. It is generally hypothesized that $C E R 1$ gene functions as aldehyde decarbonylase converting aldehydes into alkanes in the alkane pathway ${ }^{40}$. If the above 
hypothesis is accepted, the genetic mutation of this gene results in the deactivation of aldehyde decarbonylase enzyme. Therefore, in cer 1 mutant, almost no formation of alkanes is predicted and downstream ketones (Schematic Diagram), which is also reflected by the deficiency of the C29 alkane and the C29 ketone on the mutant in our results (Figure 2). The MS images for the C31 alkane $(m / z 543)$ and the C29 secondary alcohol $(\mathrm{m} / z$ 531) also showed the reduced abundance on the mutant (cer1) in comparison with the wild-type (data not shown). GC-MS data also revealed that these compounds were lower in abundance on the mutant compared to the wild-type. Alkanes are isobaric metabolites with aldehydes at the same nominal mass-to-charge $(\mathrm{m} / \mathrm{z})$ ratio so these cannot be differentiated in low mass resolution spectrometer, LTQ. For example, the peaks for ${ }^{107} \mathrm{Ag}$ adduct of $\mathrm{C} 29$ alkane and C28 aldehyde are overlapped at $\mathrm{m} / \mathrm{z} 515$, and the peaks at $\mathrm{m} / \mathrm{z} 543$ are attributed to ${ }^{107} \mathrm{Ag}$ adduct of C31 alkane and C30 aldehyde, respectively. In Figure 2, acquired with low resolution mass spectrometer, the contribution of C28 aldehyde to spatial distribution of metabolite at $\mathrm{m} / \mathrm{z} 515$ was ignored because the abundance level of the $\mathrm{C} 28$ aldehyde is much less than that of $\mathrm{C} 29$ alkane according to GC-MS data. Although our study still represents the spatial distribution of the dominant wax species well, there is a limitation in showing the individual distribution of isobaric metabolites due to paucity of mass resolving power. Therefore, the use of GC-MS data has been inevitable with low mass resolution mass spectrometer when the comparison of abundance levels is necessary for these isobaric compounds. This possible problem was resolved in HRMS (high resolution mass spectrometry) and will be mentioned in one of the following sections.

In contrast, $\mathrm{C} 30 \mathrm{FA}(\mathrm{m} / \mathrm{z} 559)$ was shown to be more abundant in the mutant than the wildtype in both MALDI MS images and GC-MS data. In Figure 2, C30 FA is sparsely distributed at low intensities on the wild-type, and barely observed only on the carpel and the tip of the petal 
while the intensity level of C30 FA is very noticeable on the mutant. It is especially obvious on the carpel and the tip of petal. In addition, minor level of C30 FA was also detected on the mutant sepal.

Unlike the C30 FA, other FAs detected do not display the higher abundance level on the mutant compared to the wild-type. For example, both the C26 FA $(\mathrm{m} / \mathrm{z} 503)$ and C28 FA $(\mathrm{m} / \mathrm{z}$ 533; $\left.\left[\mathrm{C} 28 \mathrm{FA}+{ }^{109} \mathrm{Ag}\right]{ }^{+}\right)$exist in higher abundance on the wild-type than in the mutant as the C29 alkane and/or C29 ketone whereas the GC-MS data showed reverse trend. The spatial distribution of C28 FA is concentrated mostly at the carpel and also observed on the discrete part of petal and stamen, similar to the $\mathrm{C} 29$ alkane, C29 ketone. C26 FA exhibits quite different sublocalization from the C29 alkane, C29 ketone, and C28 FA in that it is not highly concentrated at the carpel but rather distributed more abundantly on the petal. Overall, the C26 FA was distributed more uniformly over the whole flower than the other compounds shown in Figure 2. The abundances of both C26 and C28 FA are almost negligible on the mutant.

According to the simplified wax biosynthetic pathway on the paper by Samuels and coworkers ${ }^{39}$, the $\mathrm{C} 30 \mathrm{FA}$ does not have subsequent metabolic pathway other than the transformation to aldehydes whereas both C26, C28 FAs can undergo the pathway for primary alcohols and wax esters formation. Hence, the blockage to the alkane pathway can lead to the accumulation of $\mathrm{C} 30 \mathrm{FA}$ while the $\mathrm{C} 26, \mathrm{C} 28 \mathrm{FAs}$ can be diverted to primary alcohol pathway (Schematic Diagram). For this reason, C30 FA showed higher abundance on the mutant compared to the wild-type. The MS image for C32 FA ( $/ z / z 589 ;{ }^{109} \mathrm{Ag}$ adduct), which does not have an alternative pathway besides aldehyde formation, also displays that its abundance level slightly increases on the mutant as C30 FA (data not shown). 
The distribution of the $\mathrm{C} 26, \mathrm{C} 28, \mathrm{C} 30 \mathrm{FA}$ was also examined with using the colloidal graphite as a matrix instead of the colloidal silver for the consideration of matrix effect in wax detection (Figure S-1; Supplementary Figure in Appendix 2). The distribution of C26 FA unveiled by the colloidal graphite showed the consistent results with those observed with colloidal silver. The C26 FA was exhibited at higher abundance on the wild-type than the mutant, and its distribution was almost deficient especially on the carpel. However, it was mostly distributed with higher abundance on the sepal and it was also found on the discrete section of petal. From the fact that the C26 FA distribution detected with colloidal silver was more widely accumulated on the petal at high abundance level, the two matrixes produce a different effect on the ionization of the same compound. One thing to note is that the mutant sample (cer1) below the dashed line was damaged during the sample preparation. When this sample damage is taken into account, the C26 FA is almost deficient on cer 1 . This is consistent with the observation with colloidal silver. The accumulation of the C30 FA was also consistently detected with colloidal graphite. Compared to the wild-type, the most distinct accumulation was observed on the carpel of the mutant. The accumulation of the C30 FA on the carpel is also well visualized in Figure 2 where the colloidal silver was used as a matrix. Meanwhile, although the C28 FA distinctly showed its higher abundance on the wild-type than the mutant especially on the carpel and the tip of petals with colloidal silver in Figure 2, the high abundance of the C28 FA on the tip of wild-type petals was less clearly seen with colloidal graphite (Figure S-1). However, its accumulation on the carpel is still expressed well even with colloidal graphite. Although considering the artifacts caused by sample damage on the lower part of the MS image for the $\mathrm{C} 28$, the degree of difference in abundance between two genotypes was slightly less than that observed with colloidal silver, so we assumed that this difference was primarily arising from the fact that the ionization efficiency 
of the C28 FA with colloidal graphite may not as good as with colloidal silver. Accordingly, the detection of the cuticular waxes is indeed affected by the effectiveness of matrix. In addition, the different ionization efficiency of waxes can be deduced based on the suborgan-dependent intensity variation.

All three $\mathrm{C} 26, \mathrm{C} 28, \mathrm{C} 30 \mathrm{FAs}$ show higher abundance in cer 1 compared to the wild-type according to GC-MS data (Table 1). Table 1 also shows the semi-quantification of epicuticular waxes extracted from LDI-MS data. For semi-quantification of LDI-MS data, representative spectra were generated by averaging the spectra over various regions and then the signal intensity of each wax compound was divided by $m / z 216$ (silver dimer). The matrix effect on ionization is assumed to be similar between the wild-type and the mutant. As compared above, confirming the suggested wax biosynthetic pathway ${ }^{39}$, the semi-quantification results in general match well with wax abundance from GC-MS method except for some cases such as C26 and C28 FAs. This discrepancy between GC-MS and LDI-MS is attributed to the fact that sampling depth by both methods are not the same. In other words, the wax abundances revealed by LDIMS may not represent the total extraction of wax compounds from the plant. In other words, GCMS reflects the total wax abundance regardless of the consideration of their actual deposition depth.

MS Imaging of Epicuticular Waxes on the Arabidopsis Leaves. Figure 3 shows the distribution of the metabolites detected from the Arabidopsis leaves. The metabolite abundances between the wild-type and the mutant leaves show consistencies with those observed on the flowers; high abundance of the C29 alkane ( $\mathrm{m} / \mathrm{z} 515), \mathrm{C} 29$ ketone $(\mathrm{m} / \mathrm{z} 529), \mathrm{C} 26 \mathrm{FA}(\mathrm{m} / \mathrm{z} 503)$, and C28 FA $(\mathrm{m} / \mathrm{z} 533)$ in the wild-type and high abundance of the C30 FA $(\mathrm{m} / z$ 559) in cer1 (MS image of C28 FA not shown). GC-MS data for leaves are also consistent with those of flowers: 
high abundance of the C29 alkane and C29 ketone in the wild-type, high abundance of all FAs in cer1. Besides the three FAs mentioned above, the abundances of the C32 FA $(m / z 589$; [C32 FA $\left.\left.+{ }^{109} \mathrm{Ag}\right]^{+}\right)$and $\mathrm{C} 34 \mathrm{FA}\left(\mathrm{m} / \mathrm{z} 617 ;\left[\mathrm{C} 34 \mathrm{FA}+{ }^{109} \mathrm{Ag}\right]^{+}\right)$on two genotypes were also compared and it turned out that both compounds decreased on the mutant. According to Table 1, the abundance of FAs with C26, 28, 32 alkyl chains on two genotypes are inconsistent with GC-MS which exhibits higher abundance on the mutant for all three. As already mentioned earlier, the results from GC-MS are not always accordant with those seen from the MS imaging since GC-MS detects the whole wax extracts from the leaf, not only those from its outer surface layer. It is worth pointing out that although the metabolites investigated except for C30 FA have the similar trends in terms of abundance, the distribution of each wax compound is different depending on the species.

MS Imaging of Epicuticular Waxes on the Arabidopsis Stems. The Arabidopsis stems used have a length of about $4-7 \mathrm{~cm}$ in general. Because it takes a long time in acquiring the mass spectrum on each data point along this long stem, the apical part near the flower and the basal part near the root were selected for the MS imaging. Also, MS imaging can help to validate if there is any difference in the distribution of metabolites at two termini towards the flower and root.

First of all, when the abundances of the major wax compounds are compared between the two genotypes (Figure 4), stems also show the consistent results with the flowers, leaves (Figure 2, 3). For instance, the $\mathrm{C} 29$ alkane $(\mathrm{m} / \mathrm{z} 515)$ is more copious on the wild-type than the mutant for both apical and basal parts of the stem. MS images of other wax compounds such as C29 ketone $(\mathrm{m} / \mathrm{z}$ 529) and C28 FA ( $\mathrm{m} / \mathrm{z} 533$; data not shown) display the same trends whereas the C30 FA $(\mathrm{m} / \mathrm{z}$ 559) was more abundant on the mutant than the wild-type. The above observations from the MS 
imaging mostly match well with the wax abundance provided by GC-MS (Table 1) except for C26, C28 FAs, and also elucidate the preceding observations on different organs in connection with the generally hypothesized role of $C E R 1$ gene.

As shown in Figure 4, there exists a clear difference in the epicuticular wax distribution and abundance between the apical and basal part of the stem according to MS imaging. For the wildtype, all wax compounds shown in Figure 4 are more abundant on the basal part than the apical part. However, all these compounds are present in a greater quantity on the apical part than the basal part for the mutant. Although these observations were seen reproducibly in our experiments for the MS imaging, further experiments with other methods such as GC-MS need to be done for the clear verification.

MS Imaging for Each Isobaric Ion with High Mass Resolution. Although the distribution of representative wax metabolites was successfully visualized by using LTQ mass spectrometer, this low resolution mass spectrometer also showed the limitation in some cases, especially in separating the aldehydes and alkanes which are important to elucidate the functional role of mutated CER 1 gene. This was the biggest problem associated with the previous work ${ }^{6}$. The high resolution mass spectrometer was used to address this problem involving the ambiguity in peak assignment of isobaric ions.

Because both C29 alkane $(\mathrm{m} / \mathrm{z} 515.385)$ and C28 aldehyde $(\mathrm{m} / \mathrm{z} 515.348)$ appear at $\mathrm{m} / \mathrm{z} 515$ in the mass spectrum acquired with low resolution mass spectrometer, it was not possible to discriminate their spatial distribution by MS imaging. However, as shown in the spectrum of Figure 5, the separation of the two peaks having the same nominal mass $(\mathrm{m} / \mathrm{z} 515)$ was possible to see and each separated peak could be assigned to $\mathrm{C} 29$ alkane $\left(\left[\mathrm{C}_{29} \mathrm{H}_{60}+{ }^{107} \mathrm{Ag}\right]\right]^{+}, \mathrm{m} / z$ 515.3728) and $\mathrm{C} 28$ aldehyde $\left(\left[\mathrm{C}_{28} \mathrm{H}_{56} \mathrm{O}+{ }^{107} \mathrm{Ag}\right]^{+}, \mathrm{m} / z\right.$ 515.3376) with the mass accuracy of -2.5 
ppm, $-0.1 \mathrm{ppm}$, respectively from chemical composition analysis. Similarly, the isobaric ions at the nominal mass of $\mathrm{m} / \mathrm{z} 517$ can be differentially assigned to adduct of the C29 alkane with ${ }^{109} \mathrm{Ag}$ isotope $\left(\mathrm{C}_{29} \mathrm{H}_{60}{ }^{109} \mathrm{Ag}, \mathrm{m} / z\right.$ 517.3734) and the $\mathrm{C} 28$ alcohol $\left(\mathrm{C}_{28} \mathrm{H}_{58} \mathrm{O} \mathrm{Ag}, m / z\right.$ 517.3531).

Figure 5 displays the MS images with the accurate mass (0.02 Da mass window) for the isobaric ions at $m / z 515$. Based on this, it is possible to compare the abundances of each isobaric ion on the mutant with respect to the wild-type. When two MS images of the $\mathrm{C} 29$ alkane are compared, it is clearly seen that the C29 alkane dramatically decreased on the mutant. While the C29 alkane is almost absent on the mutant, it is distributed over the whole surface area with high abundance on the wild-type. On the contrary, the C28 aldehyde is almost lacking on the wildtype, but its abundance level slightly increased on the mutant. Although now shown in Figure 5, the $\mathrm{C} 30$ aldehyde $(\mathrm{m} / \mathrm{z} 543)$, the precursor of $\mathrm{C} 29$ alkane formation, was also found to display the significant increased wax level on the mutant. The product of C28 aldehyde decarbonylation, C27 alkane ( $m / z$ 487), could not be compared on both genotypes due to the high background noise. This result provides a substantial proof for the claim that the CER 1 gene plays a role in the conversion of aldehydes to alkanes in the wax biosynthetic pathway. Likewise, the isobaric ions at $m / z 543$ can be discriminated into two different wax compounds (C31 alkane, C30 aldehyde) with high resolution mass spectrometer. Alternatively, MS/MS spectrometry could be another way to separate isobaric ions, but it was not performed in our experiment because 1) it is significantly limited by mass spectrometer sensitivity, 2) it is challenging unless we have the prior idea about what metabolites exist on the sample surface, 3) it does not work for the metabolites with the long alkyl chain due to their low efficiency of fragmentation.

MS Imaging of Flavonoid Compounds on the Arabidopsis Flowers. For the test of the relevance of $C E R 1$ gene mutation to the distribution and/or abundance of flavonoids, the spatial 
distribution of flavonoid compounds on the Arabidopsis whole flowers was also studied with the colloidal graphite on the cer 1 mutant with respect to the wild-type (Figure S-1 in Appendix 2). Flavonoids are minor components of the cuticular wax together with triterpenoids, phenylpropanoids ${ }^{39}$, and the imaging of some flavonoid compounds in intact tissue by GALDI (colloidal graphite-assisted laser desorption/ionization) MS has been reported in earlier work ${ }^{43}$. Since the CER 1 gene is known to be in charge of the biosynthesis of the major wax compounds such as aldehydes, alkanes, ketones, alcohols, and esters, it is predicted that the $C E R 1$ gene mutation induces almost no significant changes in terms of abundance and/or distribution of flavonoid compounds.

The observations seen in Figure S-1 are in accordance with the preliminary expectations. For example, the genetic mutation of $C E R 1$ gene barely affects the abundance of the glycosylated flavonol, kaempferol-rhamnoside (K-Rha; $m / z$ 431). The distribution of the K-Rha on the wildtype looks similar to that on the mutant. The K-Rha mainly has an occurrence on the tip of the petal and the carpel for both genotypes. One of the flavonols, quercetin (Q; $m / z 301)$, also displays the similar trend on the wild-type and the mutant, showing almost no major changes in the abundance and spatial distribution. For both genotypes, $Q$ is distributed on the midpoint of the petal at the highest abundance level, on the carpel with the much less amounts and on the sepal with almost negligible quantity. Even though isorharmnetin (I; $\mathrm{m} / \mathrm{z} 315)$ displayed the most noticeable difference in the abundance among all flavonoids shown, the degree of changes in abundance is still much less than that of major wax compounds. Their spatial distributions are also very similar on Ler and cer1. Therefore, the MS images shown in the Figure S-1 experimentally proved that the distribution and/or abundance of flavonoids we monitored are 
irrelevant to the $C E R 1$ gene mutation which majorly affects the major wax compounds in

\section{Schematic Diagram.}

MS Imaging of Root Metabolites at High Spatial Resolution. We have a high spatial resolution which was applied to get the surface metabolites images of Arabidopsis roots of Ler versus cer 1 . Submicrometer MS imaging of potassium and calcium has been performed on pine

tree roots by Spengler and Huber ${ }^{44}$; however, the root MALDI MS imaging has been achieved for molecular species for the first time at our best knowledge.

In our experimental setup for the root MS imaging in high spatial resolution, the optical fiber with $25 \mu \mathrm{m}$ core diameter was used for the reduction of laser beam size to $\sim 12 \mu \mathrm{m}$ on the sample. The improved spatial resolution of about $\sim 12 \mu \mathrm{m}$ was achieved also with the high mass resolution for the root MS images. We failed to gain the signal from the thick part of the root at presumably because the thick root part did not allow the well-defined uniform electric field for the effective ion extraction. The nonflat thick part of the roots especially makes it hard to attach the sample flat to the surface of target plate, and therefore also results in the poor signal detection over those surface areas. This implies that the caution is necessary in quantitative interpretation because the ion signals may be affected by the distorted laser depth of field and may not represent the actual amount of analyte present on the surface.

Different types of metabolites from those on other organs of Arabidopsis have been known in the root such as caffeate, coumarate with $\mathrm{C} 18-22$ alkyl chain or sterol ${ }^{45}$ which were also observed by the MS imaging. Despite the lack of MS database for the root metabolites, the direct identification of ions detected could be performed through the chemical composition analysis due to high mass resolution. GC-MS analysis of metabolites on the root surface confirmed the high abundance of the metabolites we observed (data not shown). The two sets of MS images for 
C20 caffeate $(\mathrm{m} / \mathrm{z} 567)$ and C22 coumarate $(\mathrm{m} / \mathrm{z} 579)$ were shown in Figure 6 . Both of these metabolites show almost no significant changes in their abundances upon the CER1 gene mutation with the poor ion signal due to the thick part of roots taken into account while the epicuticular waxes were found at different abundance level on the mutant compared to the wildtype for other organs. Because the major compounds detected on the root are formed through the esterification of coumaric acid, caffeic acid with $\mathrm{C} 18-22$ saturated primary alcohols, the formation of these are not affected by the mutation of CER1 gene which is assumed as aldehyde decarbonylase in the wax biosynthetic pathway.

\section{Conclusions}

In our work, the high spatial resolution achieved by optical fibers helped to attain the detailed MS images of plant surface metabolites of interest even on the minute flower suborgan-stamen. In addition, high mass resolution spectrometry (Orbitrap) made it possible to address the vagueness in the exact identification and obtainment of the separate MS images for isobaric ions for comparison of abundance level of each isobaric ion between the mutant and the wild-type. The most prominent progress made was the differentiation of isobaric ions, for example, C29 alkane and C28 aldehyde at $\mathrm{m} / \mathrm{z} 515$ even without consecutive tandem MS and the consequent capability of generating the MS images for each. These improvements greatly contributed to the elucidation of CER 1 gene functional role in our case by visualizing the spatial distribution of aldehydes, alkanes which showed direct influences from the alteration of $C E R 1$ gene. In this manner, direct identification of wax metabolites through the chemical composition analysis as well as their detailed spatial distribution on the targeted organ tissues (flowers, leaves, stems and 
roots) by our technical improvements proved the successful application of MS imaging to a biological system. Due to the lack of mass spectral database for root metabolites, root imaging necessitated the high mass resolution for clear identification of compounds detected (data not shown) as well as high spatial resolution owing to the diminutive root tissue. Both these factors were also satisfied by our technical advances and finally root imaging was successfully performed with MALDI MS imaging for the first time. Our systematic study on various organs not only supported the claim about the functional role of $C E R 1$ gene by displaying the abundance changes of cuticular waxes on the mutant compared to the wild-type, but also allowed to observe their site-specific expression. However, even higher spatial resolution is necessary for imaging plant metabolites in subcellular scale to clearly elucidate the more detailed site-specific expression of metabolites as a result of genes mutation. With our current setup for matrix application, it was confirmed that the colloidal silver was homogeneous within the range of $\sim 3$ $10 \mu \mathrm{m}$ by SEM (scanning electron microscopy). To achieve higher spatial resolution, it is absolutely necessary to explore a better way for even more homogeneous matrix deposition in the near future. On the other hand, based on the discrepancy of abundance level between GC-MS and MS imaging for some wax compounds, it can be deduced that the detection of cuticular wax by MS imaging does not reflect the whole wax abundance on the each organ. Therefore, the profiling of the plant metabolites at different depth and their imaging by MS imaging technique could be also worth trying in the following study. 


\section{Acknowledgement}

Acknowledgement is made to Sangwon Cha for his technical advice. We also acknowledge the technical expertise and advice of Ann Perera, W. M. Keck Metabolomics Research Laboratory. The Ames Laboratory is operated for the U.S. Department of Energy by Iowa State University under Contract No. DE-AC02-07CH11358. This work was supported by U.S. Department of Energy, Office of Basic Energy Science, Division of Chemical Sciences.

\section{Appendixes}

MS images of fatty acids (C26-C30) and flavonoids on the Arabidopsis flowers obtained with colloidal graphite as a matrix and MS images of C29 alkane $(\mathrm{m} / \mathrm{z} 515)$ and C29 ketone $(\mathrm{m} / \mathrm{z} 529)$ on the Arabidopsis flowers at different spatial resolution are listed in Appendix 2. 
Table1. Semi-quantification of epicuticular wax compounds detected by LDI-MS and comparison with the GC-MS data

\begin{tabular}{|c|c|c|c|c|c|c|c|c|}
\hline \multirow{3}{*}{ Organ } & \multirow{3}{*}{ Identification } & \multirow{3}{*}{$m / z$} & \multicolumn{4}{|c|}{ LDI-MS (\%) } & \multicolumn{2}{|c|}{ GC-MS (nmol/g) } \\
\hline & & & \multicolumn{2}{|c|}{ Wild-type (Ler) } & \multicolumn{2}{|c|}{ Mutant (cer1) } & \multirow[t]{2}{*}{ Wild-type (Ler) } & \multirow[t]{2}{*}{ Mutant (cer1) } \\
\hline & & & Carpel & Petal & Carpel & Petal & & \\
\hline \multirow{5}{*}{$\begin{array}{l}\text { Flowers } \\
\text { (carpel) }\end{array}$} & C29 Alkane & 515 & $6.0 \pm 0.7$ & $0.4 \pm 0.2$ & $0.1 \pm 0.03$ & $0.02 \pm 0.007$ & $1450.0 \pm 46.2$ & $209.6 \pm 89.3$ \\
\hline & C29 Ketone & 529 & $9.6 \pm 1.2$ & $0.08 \pm 0.04$ & $0.1 \pm 0.1$ & $0.03 \pm 0.01$ & $367.9 \pm 49.8$ & $59.0 \pm 15.6$ \\
\hline & C26 FA & 503 & $0.7 \pm 0.2$ & $0.7 \pm 0.2$ & $0.03 \pm 0.003$ & $0.07 \pm 0.03$ & $2.3 \pm 1.4$ & $6.4 \pm 2.5$ \\
\hline & $\mathrm{C} 28 \mathrm{FA}^{*}$ & 533 & $5.1 \pm 1.1$ & $1.5 \pm 0.6$ & $0.7 \pm 0.01$ & $0.9 \pm 0.1$ & $0.4 \pm 0.3$ & $3.2 \pm 1.1$ \\
\hline & C30 FA & 559 & $0.4 \pm 0.06$ & $0.08 \pm 0.01$ & $0.7 \pm 0.3$ & $0.1 \pm 0.06$ & $5.5 \pm 0.8$ & $71.3 \pm 25.8$ \\
\hline \multirow{7}{*}{ Leaves } & C29 Alkane & 515 & \multicolumn{2}{|c|}{$6.3 \pm 1.6$} & \multicolumn{2}{|c|}{$1.4 \pm 0.2$} & $98.9 \pm 5.8$ & $17.1 \pm 1.1$ \\
\hline & C29 Ketone & 529 & \multicolumn{2}{|c|}{$0.5 \pm 0.09$} & \multicolumn{2}{|c|}{$0.1 \pm 0.02$} & $2.3 \pm 1.1$ & $0.3 \pm 0.1$ \\
\hline & C26 FA & 503 & \multicolumn{2}{|c|}{$6.0 \pm 2.3$} & \multicolumn{2}{|c|}{$1.2 \pm 0.2$} & $20.1 \pm 2.0$ & $96.0 \pm 4.8$ \\
\hline & $\mathrm{C}^{28} \mathrm{FA}^{*}$ & 533 & \multicolumn{2}{|c|}{$2.7 \pm 0.2$} & \multicolumn{2}{|c|}{$1.2 \pm 0.2$} & $3.5 \pm 0.3$ & $9.5 \pm 0.9$ \\
\hline & C30 FA & 559 & \multicolumn{2}{|c|}{$0.4 \pm 0.08$} & \multicolumn{2}{|c|}{$0.3 \pm 0.08$} & $5.6 \pm 1.4$ & $33.2 \pm 0.5$ \\
\hline & C32 FA ${ }_{*}^{*}$ & 589 & \multicolumn{2}{|c|}{$0.4 \pm 0.3$} & \multicolumn{2}{|c|}{$0.1 \pm 0.04$} & $19.2 \pm 4.5$ & $25.6 \pm 2.5$ \\
\hline & C34 FA ${ }^{*}$ & 617 & \multicolumn{2}{|c|}{$0.5 \pm 0.3$} & \multicolumn{2}{|c|}{$0.06 \pm 0.02$} & - & - \\
\hline \multirow{8}{*}{ Stems } & & & Apical & Basal & Apical & Basal & & \\
\hline & C29 Alkane & 515 & $61.8 \pm 9.0$ & $53.1 \pm 10.1$ & $18.7 \pm 3.5$ & $9.6 \pm 2.5$ & $1447.9 \pm 206.7$ & $52.1 \pm 5.3$ \\
\hline & C29 Ketone & 529 & $231.5 \pm 34.3$ & $166.2 \pm 58.3$ & $43.2 \pm 7.1$ & $12.8 \pm 3.8$ & $850.2 \pm 143.3$ & $64.0 \pm 2.2$ \\
\hline & C26 FA & 503 & $8.6 \pm 1.3$ & $4.6 \pm 1.3$ & $2.5 \pm 0.6$ & $3.1 \pm 2.3$ & $0.06 \pm 0.01$ & $0.14 \pm 0.02$ \\
\hline & $\mathrm{C} 28 \mathrm{FA}^{*}$ & 533 & $62.3 \pm 8.4$ & $43.8 \pm 14.3$ & $11.2 \pm 2.9$ & $7.3 \pm 2.8$ & $0.1 \pm 0.01$ & $0.1 \pm 0.02$ \\
\hline & C30 FA & 559 & $19.2 \pm 3.3$ & $19.2 \pm 3.3$ & $58.5 \pm 21.1$ & $21.0 \pm 5.9$ & $0.3 \pm 0.05$ & $1.7 \pm 0.4$ \\
\hline & C32 FA ${ }^{*}$ & 589 & $5.6 \pm 1.4$ & $4.0 \pm 1.9$ & $4.4 \pm 2.0$ & $3.8 \pm 2.4$ & - & - \\
\hline & C34 FA ${ }^{*}$ & 617 & $5.4 \pm 1.1$ & $2.4 \pm 1.0$ & $1.0 \pm 0.7$ & $2.5 \pm 2.2$ & - & - \\
\hline
\end{tabular}

For the cuticular wax compounds shown on the figures, their relative abundance was calculated by dividing the signal intensity of each wax metabolite by $\mathrm{m} / \mathrm{z} 216$ (silver dimer) on the representative mass spectrum of each organ which was generated by averaging the spectra over the certain region of the sample with substantial intensity. All $\mathrm{m} / \mathrm{z}$ values were taken as ${ }^{107} \mathrm{Ag}$ adduct of 
each wax metabolite except C28 FA ${ }^{*}(\mathrm{~m} / \mathrm{z} 533)$ due to its overlap with ${ }^{109} \mathrm{Ag}$ adduct ion of C29 ketone $(\mathrm{m} / \mathrm{z}$ 529). GC-MS results were the average of three replicates with \pm standard deviation. 


\section{References}

1. Caprioli, R. M.; Farmer, T. B.; Gile, J., Anal. Chem. 1997, 69 (23), 4751-60.

2. Reyzer, M. L.; Hsieh, Y.; Ng, K.; Korfmacher, W. A.; Caprioli, R. M., J. Mass Spectrom. 2003, 38 (10), 1081-92.

3. $\quad$ Stoeckli, M.; Chaurand, P.; Hallahan, D.; Caprioli, R., Nature Medicine 2001, 7 (4), 493496.

4. Stoeckli, M.; Staab, D.; Staufenbiel, M.; Wiederhold, K. H.; Signor, L., Anal. Biochem. 2002, $311(1), 33-9$.

5. $\quad$ Rohner, T. C.; Staab, D.; Stoeckli, M., Mech. Ageing Dev. 2005, 126 (1), 177-85.

6. $\quad$ Cha, S.; Song, Z.; Nikolau, B. J.; Yeung, E. S., Anal. Chem. 2009, 81 (8), 2991-3000.

7. Burrell, M.; Earnshaw, C.; Clench, M., J. Exp. Bot. 2007, 58 (4), 757-63.

8. Hölscher, D.; Shroff, R.; Knop, K.; Gottschaldt, M.; Crecelius, A.; Schneider, B.; Heckel, D. G.; Schubert, U. S.; Svatos, A., Plant J 2009, 60 (5), 907-18.

9. Jun, J.; Song, Z.; Liu, Z.; Nikolau, B.; Yeung, E.; Lee, Y., Analytical Chemistry 2010, 82 (8), 3255-3265.

10. Spengler, B.; Hubert, M., J Am Soc Mass Spectr 2002, 13 (6), 735-748.

11. Chaurand, P.; Schriver, K. E.; Caprioli, R. M., J. Mass Spectrom. 2007, 42 (4), 476-89.

12. Chaurand, P.; Angle, P. M.; Caprioli, R. M., Proc. 57th ASMS Conf. on Mass Spectrometry and Allied Topics, Philadelphia, PA, May 31-June 4. 2009, Oral session MOAam10:10.

13. Qiao, H.; Piyadasa, G.; Spicer, V.; Ens, W., Int. J. Mass Spectrom. 2009, 281 (1-2), 4151.

14. Hensel, R. R.; King, R. C.; Owens, K. G., Rapid Communications in Mass Spectrometry 1997, 11 (16), 1785-1793.

15. Kruse, R.; Sweedler, J. V., Journal of the American Society for Mass Spectrometry 2003, $14(7), 752-759$.

16. Aerni, H. R.; Cornett, D. S.; Caprioli, R. M., Analytical chemistry 2006, 78 (3), 827-834.

17. Schwartz, S. A.; Reyzer, M. L.; Caprioli, R. M., J Mass Spectrom 2003, 38 (7), 699-708. 
18. Hankin, J. A.; Barkley, R. M.; Murphy, R. C., J Am Soc Mass Spectr 2007, 18 (9), 16461652.

19. Chen, Y. F.; Allegood, J.; Liu, Y.; Wang, E.; Cachon-Gonzalez, B.; Cox, T. M.; Merrill, A. H.; Sullards, M. C., Anal. Chem. 2008, 80 (8), 2780-2788.

20. Sluszny, C.; Yeung, E. S.; Nikolau, B. J., J. Am. Soc. Mass Spectrom. 2005, 16 (1), $107-$ 15.

21. Xia, Y. J.; Nikolau, B. J.; Schnable, P. S., Plant Physiology 1997, 115 (3), 925-937.

22. Koornneef, M.; Hanhart, C. J.; Thiel, F., Journal of Heredity 1989, 80 (2), 118-122.

23. Aarts, M. G.; Keijzer, C. J.; Stiekema, W. J.; Pereira, A., Plant Cell 1995, 7 (12), $2115-$ 27.

24. Jenks, M. A.; Tuttle, H. A.; Eigenbrode, S. D.; Feldmann, K. A., Plant Physiology 1995, $108(1), 369-377$.

25. Jenks, M. A.; Rashotte, A. M.; Tuttle, H. A.; Feldmann, K. A., Plant Physiology 1996, $110(2), 377-385$.

26. Jenks, M. A.; Tuttle, H. A.; Feldmann, K. A., Phytochemistry 1996, 42 (1), 29-34.

27. Lemieux, B., Trends in Plant Science 1996, 1 (9), 312-318.

28. Xia, Y. J.; Nicolau, B. J.; Schnable, P. S., Plant Cell 1996, 8 (8), 1291-1304.

29. Rashotte, A. M.; Jenks, M. A.; Nguyen, T. D.; Feldmann, K. A., Phytochemistry 1997, 45 (2), 251-255.

30. Rashotte, A. M.; Jenks, M. A.; Ross, A. S.; Feldmann, K. A., Planta 2004, 219 (1), 5-13.

31. Rashotte, A. M.; Jenks, M. A.; Feldmann, K. A., Phytochemistry 2001, 57 (1), 115-123.

32. Goodwin, S. M.; Rashotte, A. M.; Rahman, M.; Feldmann, K. A.; Jenks, M. A., Phytochemistry 2005, 66 (7), 771-780.

33. Nawrath, C., Current Opinion in Plant Biology 2006, 9 (3), 281-287.

34. Kunst, L.; Samuels, A. L., Prog Lipid Res 2003, 42 (1), 51-80.

35. Taban, I. M.; Altelaar, A. F.; van der Burgt, Y. E.; McDonnell, L. A.; Heeren, R. M.; Fuchser, J.; Baykut, G., J Am Soc Mass Spectrom 2007, 18 (1), 145-51.

36. Taban, I. M.; Altelaar, A. F.; van der Burgt, Y. E.; McDonnell, L. A.; Heeren, R. M.; Fuchser, J.; Baykut, G., J. Am. Soc. Mass Spectrom. 2007, 18 (1), 145-51.

37. Cornett, D. S.; Frappier, S. L.; Caprioli, R. M., Analytical chemistry 2008, 80 (14), 56485653. 
38. Hsieh, Y.; Casale, R.; Fukuda, E.; Chen, J. W.; Knemeyer, I.; Wingate, J.; Morrison, R.; Korfmacher, W., Rapid Commun Mass Sp 2006, 20 (6), 965-972.

39. Samuels, L.; Kunst, L.; Jetter, R., Annu. Rev. Plant Biol. 2008, 59, 683-707.

40. Jurchen, J. C.; Rubakhin, S. S.; Sweedler, J. V., J Am Soc Mass Spectr 2005, 16 (10), 1654-1659.

41. Mcnevin, J. P.; Woodward, W.; Hannoufa, A.; Feldmann, K. A.; Lemieux, B., Genome 1993, $36(3), 610-618$.

42. Hannoufa, A.; Mcnevin, J.; Lemieux, B., Phytochemistry 1993, 33 (4), 851-855.

43. Cha, S.; Zhang, H.; Ilarslan, H. I.; Wurtele, E. S.; Brachova, L.; Nikolau, B. J.; Yeung, E. S., Plant J. 2008, 55 (2), 348-60.

44. $\quad$ Spengler, B.; Hubert, M., J. Am. Soc. Mass Spectrom. 2002, 13 (6), 735-48.

45. Li, Y. H.; Beisson, F.; Ohlrogge, J.; Pollard, M., Plant Physiol 2007, 144 (3), 1267-1277. 


\section{Figure Captions}

Schematic Diagram. Simplified cuticular wax biosynthetic pathway of Arabidopsis thaliana.

Figure 1. Comparison of MS images of silver adduct of C29 alkane $(\mathrm{m} / \mathrm{z} 515)$ on an Arabidopsis whole flower (wild-type; Ler) normalized to the silver dimer, $\left[{ }^{107} \mathrm{Ag}+{ }^{109} \mathrm{Ag}\right]^{+}(\mathrm{m} / z$ 216), obtained with different spatial resolution. The MS images were acquired at the spatial resolution of $50 \mu \mathrm{m}$ with an actual laser spot size of $100 \mu \mathrm{m}$ using oversampling method (left) ${ }^{*}, 50 \mu \mathrm{m}$ with a laser spot size of $50 \mu \mathrm{m}$ (middle), $12 \mu \mathrm{m}$ with a laser spot size of $12 \mu \mathrm{m}$ (right). Although the first two MS images were both acquired with a raster size of $50 \mu \mathrm{m}$, the second MS image where the actual laser spot size is the same as the raster size used reveals minute features of the flower such as stamen and anther more in detail. As seen in this comparison, the higher spatial resolution we achieve in MS imaging, the more detailed information we can attain from the MS images acquired in terms of the spatial distribution of analytes of interest.

Figure 2. MS imaging of representative epicuticular wax compounds on the Arabidopsis flowers. The flowers used were collected on the 45th day. The selected area was scanned with a raster size of $50 \mu \mathrm{m}$. Due to the different ionization efficiency of each wax compound, the comparison of abundance levels of wax compounds was restricted to only the same species between two genotypes. The improved spatial resolution allowed the detailed MS imaging of metabolites even on the stamen, a diminutive suborgan of flower. Each epicuticular wax displays the different spatial distribution.

\footnotetext{
* Reprint with permission from Analytical Chemistry 2009, 81(8), 2991-3000 Copyright (C) 2009 American Chemical Society
} 
Figure 3. MS imaging of representative epicuticular wax compounds on the Arabidopsis leaves. The leaves used were collected on the 14th day. The selected area was scanned with a raster size of $50 \mu \mathrm{m}$. While the abundance level of C29 alkane ( $\mathrm{m} / \mathrm{z} 515), \mathrm{C} 29$ ketone $(\mathrm{m} / \mathrm{z} 529), \mathrm{C} 26 \mathrm{FA}$ ( $m / z 503)$, is higher on the wild-type (Ler) than the mutant (cer 1$)$, C30 FA ( $m / z 559)$ shows the opposite trend. The different distribution of each wax metabolite is visualized well by MS imaging.

Figure 4. MS imaging of representative epicuticular wax compounds on the Arabidopsis stems. The stems used were collected on the 28th day. For the basal part, the dimensions of the image was $1200 \mu \mathrm{m}$ (width) $\times 900 \mu \mathrm{m}$ (height) for Ler and $500 \mu \mathrm{m}$ (width) $\times 1100 \mu \mathrm{m}$ (height) for cer1, respectively. The samples were scanned with a step size of $50 \mu \mathrm{m}$. Two end parts of the stem were selected for MS imaging due to the long time consumption of MS imaging process for the whole stem which has a length of about 4-7 cm in general. The wax abundance levels between wild-type (Ler) and cer 1 were consistently observed on the stem as flowers, leaves. All representative epicuticular wax compounds display the higher abundance level on the wild-type with respect to the mutant (cer 1$)$. The different distribution of metabolites along the stem was also observed- the basal is shown to have more wax material than the apical part in the case of wild-type whereas the reverse trend was observed for the mutant (cer1).

Figure 5. Differentiation of isobaric ions with high resolution mass spectrometry. On the mass spectrum of the 28 days old Arabidopsis stem, the peaks for plant surface metabolites of interest are placed in the range of $m / z 500-600$. Isobaric ions at $\mathrm{m} / z 515$ and $\mathrm{m} / \mathrm{z} 517$ are shown. Based on the chemical composition analysis with high mass accuracy, higher peak at $m / z 515$ is assigned to $\mathrm{C} 29$ alkane $\left(\left[\mathrm{C}_{29} \mathrm{H}_{60} \mathrm{Ag}\right]^{+}\right)$with mass accuracy of $-2.5 \mathrm{ppm}$ and lower peak was assigned to 
$\mathrm{C} 28$ aldehyde $\left(\left(\left[\mathrm{C}_{28} \mathrm{H}_{56} \mathrm{OAg}\right]^{+}\right)\right.$with mass accuracy of $-0.1 \mathrm{ppm}$. For the two peaks at $\mathrm{m} / z 517$, higher peak is assigned to ${ }^{109} \mathrm{Ag}$ adduct of $\mathrm{C} 29$ alkane $\left(\left[\mathrm{C}_{29} \mathrm{H}_{60}{ }^{109} \mathrm{Ag}\right]^{+} ;-0.6 \mathrm{ppm}\right)$ and the other peak corresponds to the $\mathrm{C} 28$ alcohol $\left(\left[\mathrm{C}_{28} \mathrm{H}_{58} \mathrm{OAg}\right]^{+} ;-0.5 \mathrm{ppm}\right)$. The high mass resolving power of Orbitrap made it possible to differentiate isobaric ions, of which identification was only possible by tandem MS with low resolution MS. The MS images of differentiated isobaric ions are also displayed. The samples were scanned with a step size of $100 \mu \mathrm{m}$. It is confirmed that the C29 alkane dramatically decreased on the mutant whereas the C28 aldehyde slightly increased on the mutant compared to the wild-type. These changes in abundance of aldehyde and alkane corroborate the functional role of $C E R 1$ gene known as aldehyde decarbonylase in cuticular wax biosynthesis.

Figure 6. MS imaging of representative surface lipid metabolites on the Arabidopsis roots. Root samples were collected on the 46th day. The MS images were obtained with $12 \mu \mathrm{m}$ spatial resolution. While difference abundances in epicuticular lipids such as long chain alkanes, ketones, and fatty acids were shown on the other organs (flowers, leaves, stems) of cer 1 mutant subject to MS imaging compared to the wild-type, there is not any noticeable difference between MS images of surface lipid metabolites detected on the root (suberins) between wild-type and cer1 mutant. 


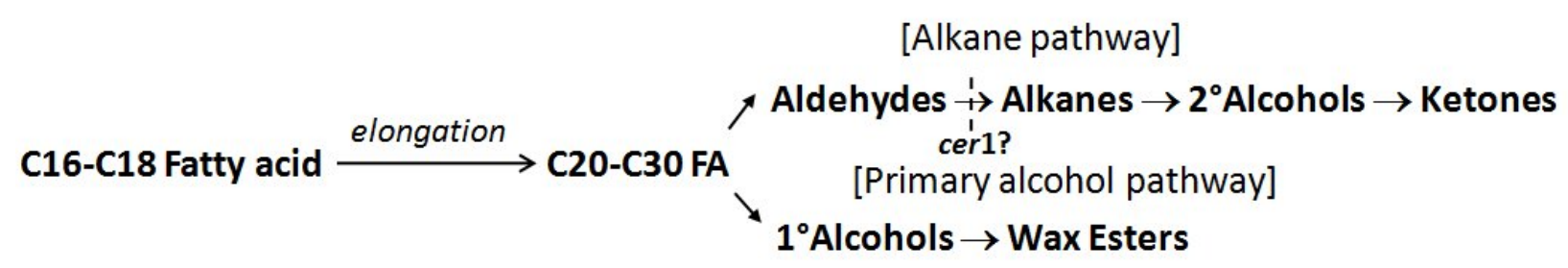

Schematic Diagram. 


\section{Arabidopsis flower wild-type (Ler)}

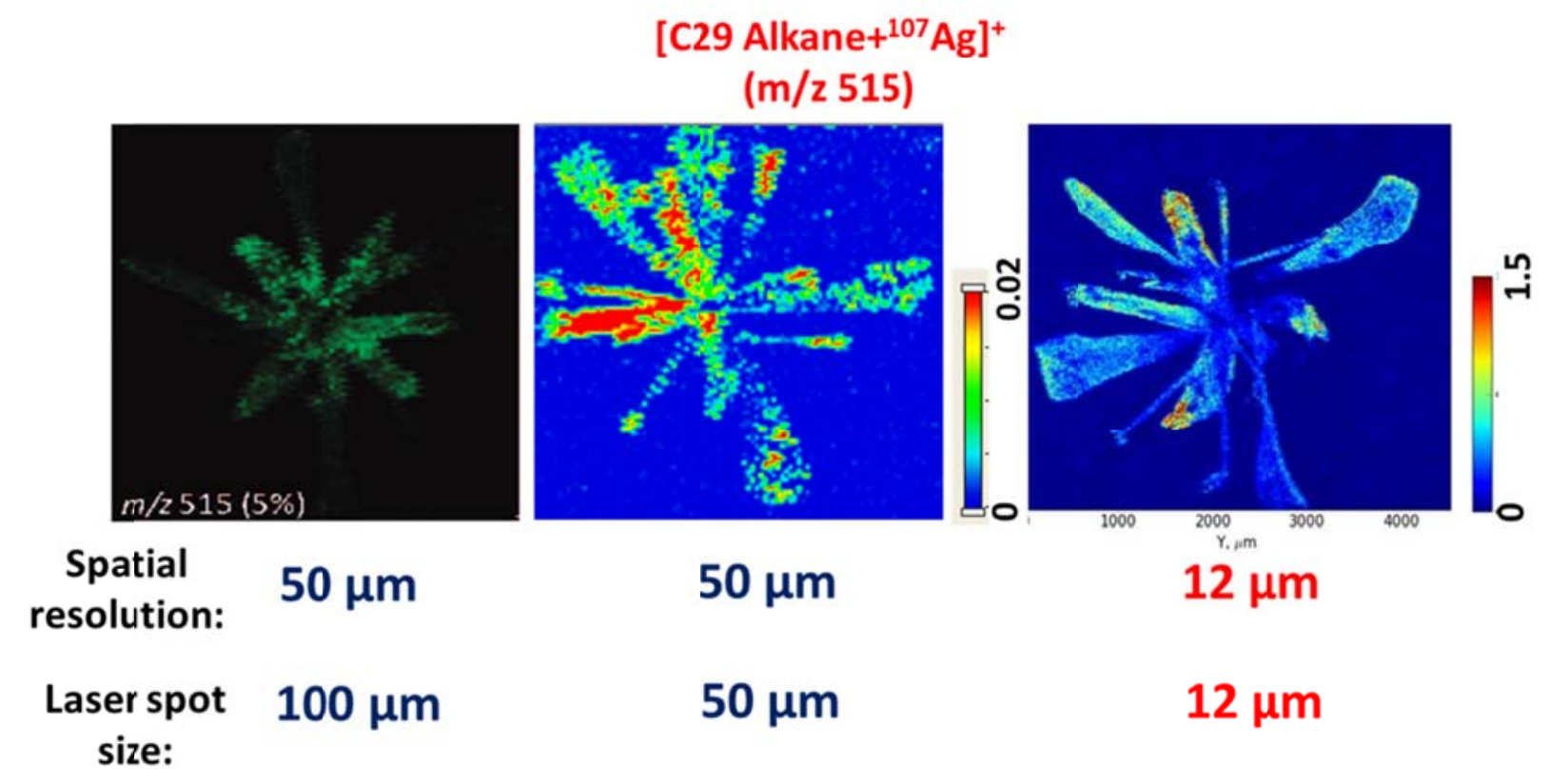

Figure 1. 


\section{[ Signal Intensity/ (m/z 216) ]}

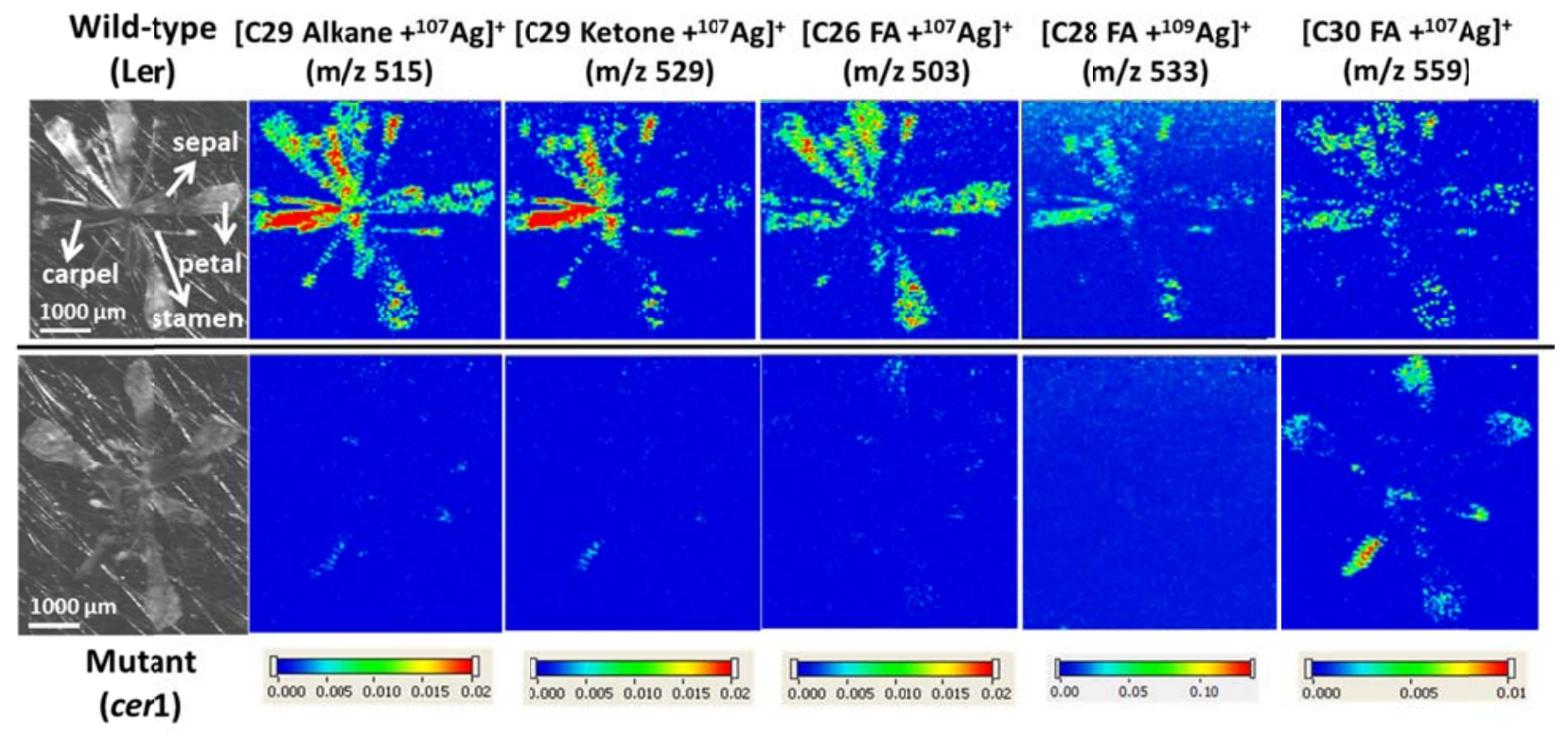

Figure 2. 
[ Signal Intensity/ (m/z 216) ]

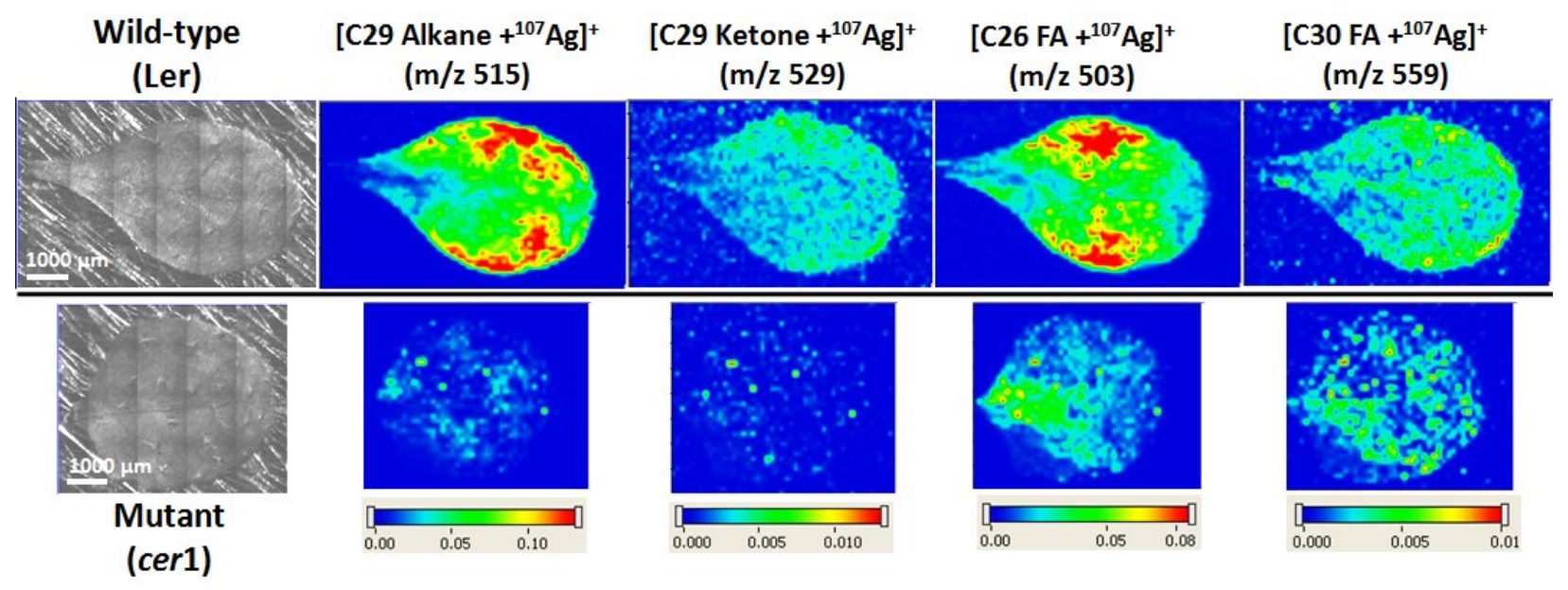

Figure 3. 


\section{[ Signal Intensity/ (m/z 216) ]}

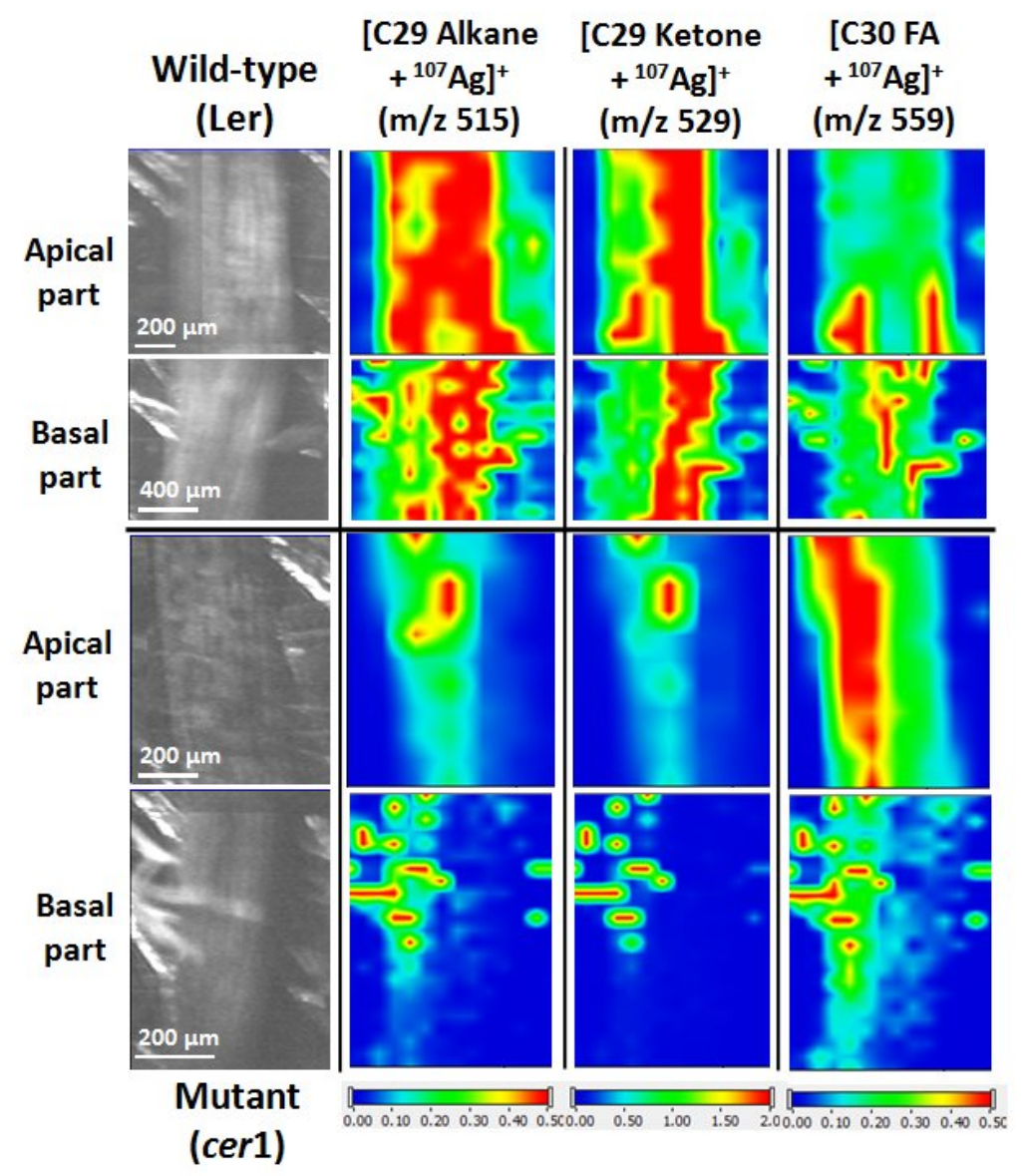

Figure 4. 
[ Signal $(\mathrm{m} / \mathrm{z} 515) /(\mathrm{m} / \mathrm{z} 216)$ ]
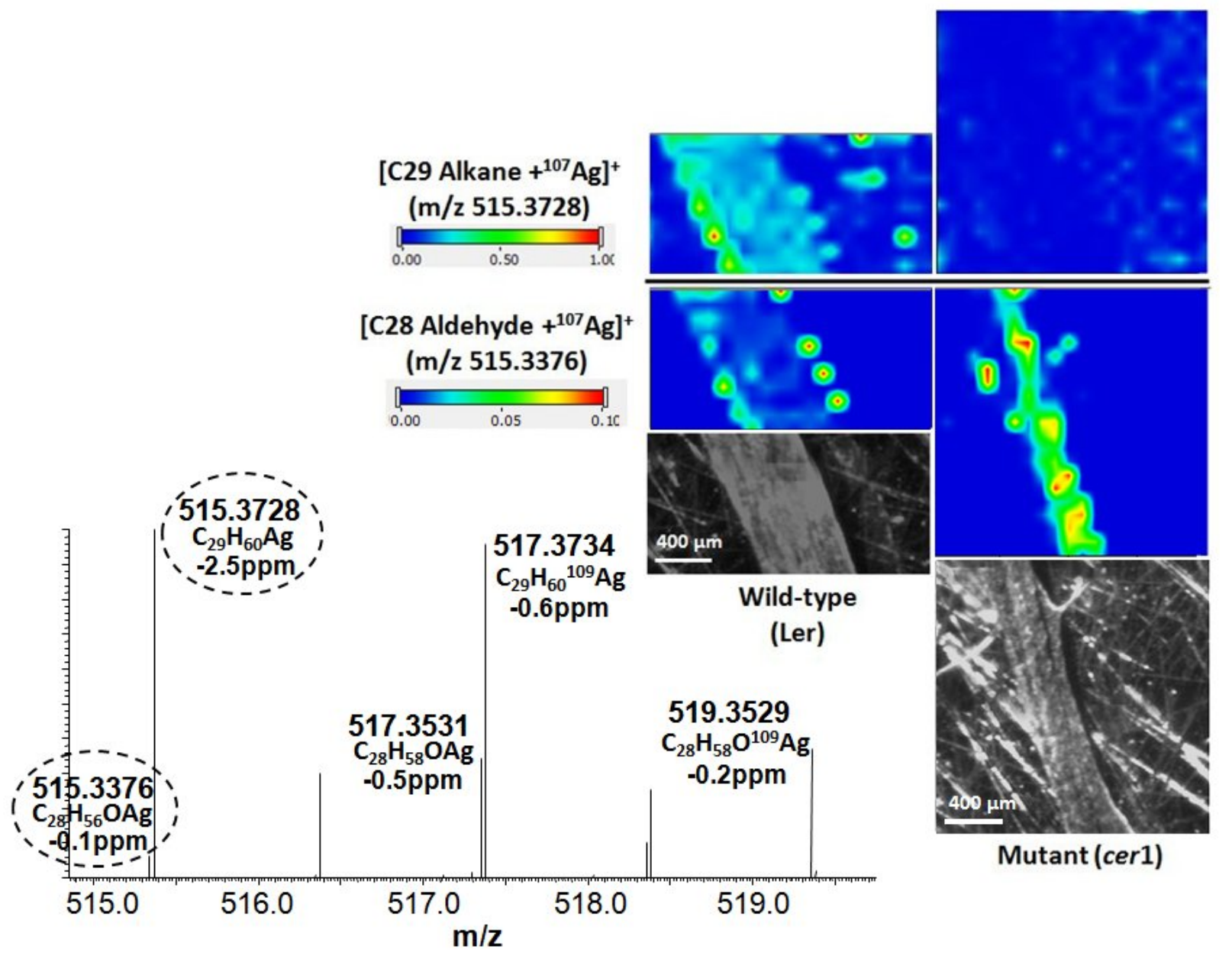

Figure 5. 


\section{[ Signal Intensity/ TIC ]}

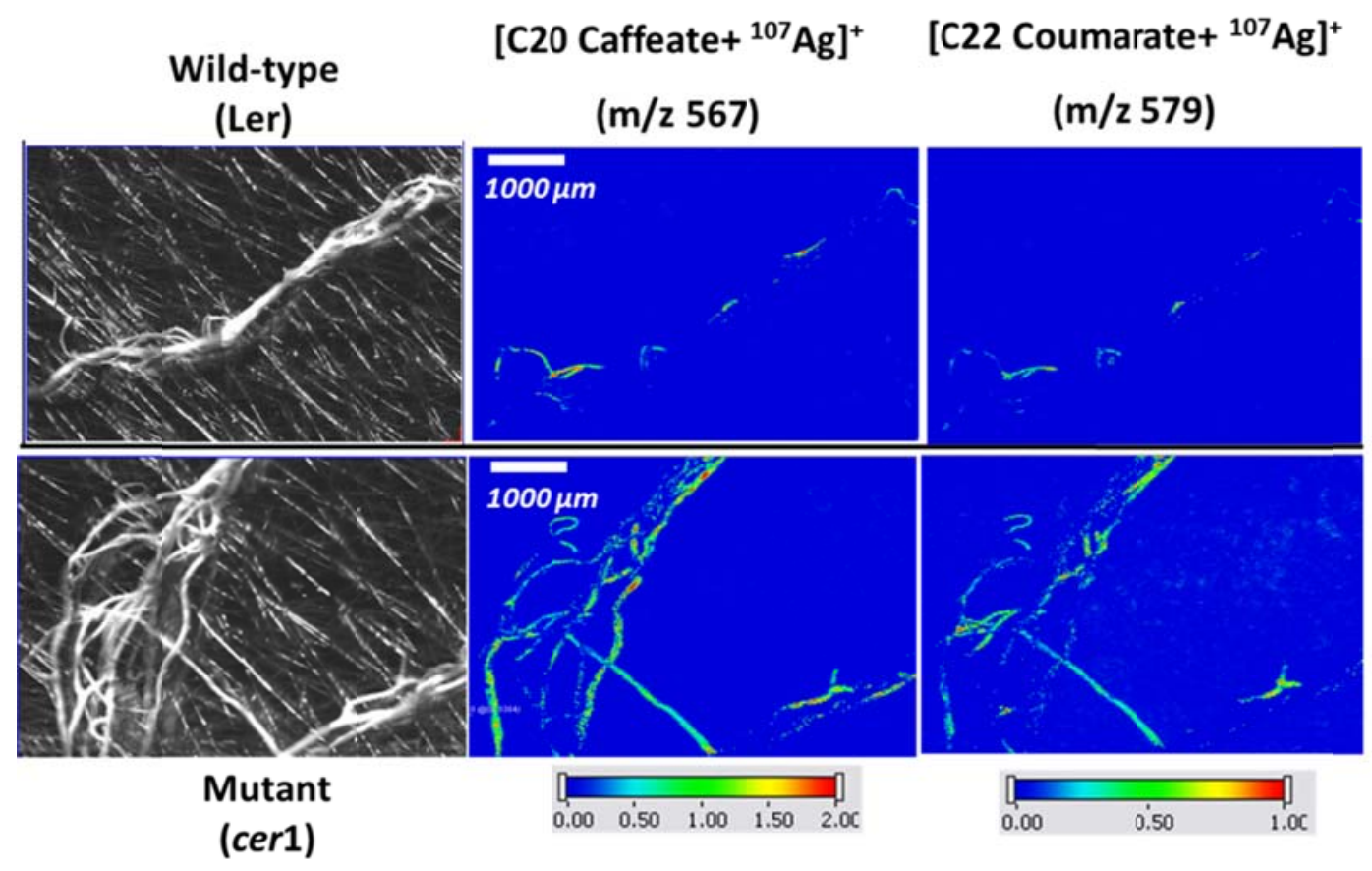

Figure 6. 


\title{
CHAPTER 4. ENZYMATIC REMOVAL OF CELL WALLS OF THE INTERNAL CELLS EXPOSED THROUGH VIBRATOME SECTIONING AND MASS SPECTROMETRIC IMAGING OF INTERNAL CELL METABOLITES
}

Ji Hyun Jun, Harry T. Horner, Basil J. Nikolau, and Young Jin Lee

\begin{abstract}
The distribution of the surface metabolites on various organs of Arabidopsis thaliana has been successfully visualized previously by developing the high-spatial and high-mass resolution laser desorption ionization (LDI)-mass spectrometric (MS) imaging. In this study, the scope of analytes is further directed toward internal metabolites. For profiling and imaging of internal cell metabolites on the plant tissue by MS imaging, vibratome sectioning of the leaf tissues was first performed to expose the cells internal to the epidermises, and then the enzymatic treatment was subsequently performed on the vibratome sections to digest the primary cell walls and middle lamella. This process exposes the internal metabolites of the naked cell protoplasts. The subsequent MS imaging on the vibratome sections digested with enzyme mixture allowed us to reveal the spatial distribution of internal metabolites, linolenic acid (C18:3 FA) and linoleic acid (C18:2 FA), heterogeneously distributed along the longitudinal section.
\end{abstract}




\section{Introduction}

The cell wall functions as a structural support and protection for the protoplasts and has fiberglass-like structure. The primary wall of growing cells is typically a thin, flexible layer (0.1$1 \mu \mathrm{m}$ ) which is primarily composed of complex polysaccharides and a small amount of structural proteins ${ }^{1}$. The main classes of polysaccharides in the cell wall are celluloses, hemicelluloses, and pectins (Figure 1). The crystalline cellulose microfibrils are linked together by non-covalent interactions with matrix polysaccharides that are hemicelloses and pectins. Hemicelluloses form a strong yet resilient network by binding to celluloses, and pectins form hydrated gels which glue adjacent cells together in an adhesive layer, the middle lamella ${ }^{1}$. The outer part of primary cell walls of many aerial organs of plants such as leaves are covered with a protective hydrophobic film $(0.1-10 \mu \mathrm{m})$ of wax and cutin, the components of plant cuticle ${ }^{2,3}$.

The existence of cutin and cell walls is a main hurdle in performing a depth profiling of the endogenous molecules directly on the intact plant tissue. Cuticular wax of the cuticle is solventsoluble lipids ${ }^{3}$, which can be removed by extensive extraction with organic solvents such as chloroform ${ }^{4,5}$. Cutin is the insoluble polyester consisting of hydroxy and epoxy fatty acids ${ }^{6}$. The removal of cell walls is also ensured only by the digestion with an enzyme mixture which degrades the components of the cell walls. Therefore, several other strategies have been previously attempted as alternatives for depth profiling.

The spatial distribution of flavonoids, typically intracellular metabolites, placed relatively at the deeper layer than the surface metabolites such as epicuticular waxes have been previously imaged on the leaf tissue of Arabidopsis thaliana by dipping of the tissue into the organic solvent for 30-60 s to dissolve the surface wax layer followed by GALDI (colloidal graphite-assisted laser desorption /ionization) mass spectrometry (MS) ${ }^{5}$. In this way, the secondary metabolites 
such as kaempferol and kaempferol rhamnoside could be successfully profiled and imaged. Similarly, serial scanning of the same surface area with multiple shots of the high-power laser with reapplication of matrix in between also allowed us to remove the outermost surface layer at the first scan and reveal the localization of kaempferol, minor components of cuticular waxes ${ }^{7}$, on the Arabidopsis stem on the second scan ${ }^{8}$.

Two approaches mentioned above successfully removed the outermost layer of the surface and allowed the profiling and mapping of the endogenous molecules placed at the inner layer of the tissue than the surface epicuticular lipids by LDI-MS. However, these two approaches are still limited to screen the metabolites only on the surface cuticle layer due to the restrictions of the degrees of removal of tissue layers or probing depth. In addition, even as a means to examine the plant metabolites possibly existing in the deeper tissue layers by LDI-MS imaging, there are also some drawbacks for each method. First, in organic-solvent dipping methods, dissolution of surface wax layer using organic solvent may result in the dissolution and/or delocalization of the targeted metabolites if the time duration of dipping process is not optimized or sample-to-sample variation in terms of size of the sample tissue is large even for the same organ. This leads to significant artifacts in terms of intrinsic spatial distribution of endogenous molecules detected. In addition, the tissue morphology can be altered or destroyed substantially after dipping process in the organic solvent if the tissue is too soft. As a result, the sample attachment onto the target plate may be disturbed, which leads to the significant difficulty in the sample preparation step for MS imaging. Second, the evident disadvantage of depth resolved MS imaging with high-power laser ablation is a long data acquisition time since hundreds of laser shots are necessary to physically remove the surface wax layer at its first scan throughout the sample. In addition, the matrix needs to be reapplied between the ablation steps to achieve efficient ionization of any 
remaining analyte in the sample and this procedure also increases the total amount of experimental time. In addition, there is a previous report that the depth of crater created by laser ablation is only $\sim 1 \mu \mathrm{m}$ or more depending on laser fluence ${ }^{9}$. It means that our probing depth is much shallower than the thickness of the epicuticular wax layer of the tissue. Moreover, the sputtering rate may not be consistent over the specimen surface.

In the present work, the in situ MS profiling and imaging of internal cellular metabolites beneath the cuticle and the cell wall layers are pursued by combining the two successive experimental protocols for different purposes: (1) vibratome sectioning of the leaf tissues to physically bypass the surface cuticle layer; and (2) treatment of the vibratome sections with the enzyme cocktail which effectively degrade the components of the primary cell walls and middle lamellae of the internal cells (Figure 1). Unlike the alternative strategies for depth profiling mentioned above, our approach allows the direct depth profiling at much deeper layers of the tissue with no difficulty associated with the presence of cutin and cell walls in probing the endogenous molecules. These successive procedures expose the internal cell protoplasts and make it possible to probe the spatial distribution of metabolites easily by the subsequent MS imaging.

For obtaining sections through the leaf, a vibratome was used instead of a cryostat to better maintain the morphology of the cut sections and avoid the chances of contamination and addition of an unnatural substrate caused by the cryo-embedding material (OCT). In addition, when the sections are prepared using the vibratome, there is less chance of artifact in terms of spatial distribution of internal metabolites which may be caused by freezing because the plant cell vacuole contains mostly water ${ }^{10}$. This spatial distribution can possibly be affected by the low temperature at which the cutting process is performed in the cryostat. 
The established standard protocol for isolation of protoplasts ${ }^{11}$ was adapted for the purpose of eliminating the protective cell wall but still maintaining the intact cell membranes, their protoplasts, and cell location within the leaf vibratome section. After careful digestion of the components of cell wall with an enzyme mixture, the exposed protoplasts were subsequently subjected to matrix application and MS imaging where two successive laser scans were performed on each data point of the sections imaged.

For MS imaging, colloidal silver was used as a matrix for several reasons. First, it does not crystalize and forms a homogeneous surface layer, and hence allows good reproducibility ${ }^{12}$. Second, this matrix makes it easier to distinguish metabolite peaks from the background peaks

by forming the intact silver $\left({ }^{107} \mathrm{Ag},{ }^{109} \mathrm{Ag}\right)$ adduct ions. Moreover, the peaks arising from matrix do not interfere with the peaks attributed to internal cell metabolites detected on the mass spectrum, which has been a significant problem for other organic matrixes in the analysis of small molecules $(<500 \mathrm{Da})$.

The enzymatic treatment of the vibratome section successfully allowed us to detect and map the localization of the internal metabolites, linolenic acid (C18:3 FA) and linoleic acid (C18:2 FA) by MS imaging using colloidal silver as a matrix. As control experiments, the spatial abundances of these metabolites were monitored on the vibratome sections which were not subjected to enzymatic digestion.

\section{Experimental Section}

Chemicals. Cellulase Onozuka R10 from Trichoderma viride (cellulase activity $>10,000$ units/g) and Macerozyme R10, a mixture of pectinase, cellulase, and hemicellulase (> 3000 units/g), were purchased from PhytoTechnology Laboratories (Overland Park, KS). Calcium 
chloride (anhydrous, suitable for plant cell culture), MES (4-morpholinoethanesulfonic acid, $\geq$ 99\%), and D-sorbitol ( $\geq 99.5 \%$ ) were obtained from Sigma-Aldrich (St. Louis, MO). Potassium hydroxide was purchased from Fisher Scientific (Fair Lawn, NJ). Colloidal silver (99.99\% pure silver, $0.65 \mathrm{~nm} ; 20 \mathrm{ppm}$ ) was purchased from Purest Colloids, Inc. (Westampton, NJ). Doubledeionized water was obtained from a MilliQ water purification system (Framington, MA).

Plant Growth Condition. Arabidopsis thaliana ecotype Columbia (Col-0) was obtained from the Arabidopsis Biological Resource Center (Columbus, $\mathrm{OH})$. Seeds were sterilized and sown on Murashige and Skoog basal salt mixture (MS) media in Petri dishes. The dishes were placed in the growth room for 10 days after breaking seed dormancy by maintaining them at $4^{\circ} \mathrm{C}$ for four days. On the $15^{\text {th }}$ day, the seedlings were transferred to soil in $5 \mathrm{~cm} \times 5 \mathrm{~cm}$ pots for continuous growth. Leaf samples were sectioned with a vibratome (Ted Pella, Redding, CA) on the 43th day for the sample preparation for MS imaging. The growth room was set at $24^{\circ} \mathrm{C}$ with continuous illumination at $85 \mu \mathrm{E} \mathrm{m}^{-2} \mathrm{~s}^{-1}$ and ambient relative humidity.

Sample Preparation for LDI-MS Imaging. The leaf tissue was collected and immediately subjected to sectioning using a vibratome. The vibratome sections were cut $80 \mu \mathrm{m}$ thick and loaded onto superfrost microscope slides (Fisher, Pittsburgh, PA) for LDI-MS imaging. The prepared sections were first dried under a moderate vacuum ( $\sim 50$ Torr) for 20 min to minimize the metabolite turnover and then one section was subsequently incubated in a Petri dish for 20 min at $37^{\circ} \mathrm{C}$ with cell wall-degrading enzyme mixture mainly composed of $2 \mathrm{wt} \%$ Cellulase Onozuka R10 and $0.3 \mathrm{wt} \%$ Macerozyme R10 in the solution containing a $1 \mathrm{mM}$ calcium chloride, 5 mM MES-KOH (pH 5.5) and $500 \mathrm{mM}$ D-sorbitol ${ }^{11,13,14}$. The incubation time was determined by observing the vibratome sections at various times with respect to minimal disintegration using bright-field microscopy. During the incubation, a Petri dish was used as a humidity chamber to 
prevent the accelerated drying of the enzyme mixture due to the warm temperature and the deactivation of enzyme. The digested vibratome sections were washed with the solution containing a $1 \mathrm{mM}$ calcium chloride, $5 \mathrm{mM}$ MES-KOH (pH 6.0) and $500 \mathrm{mM}$ D-sorbitol, osmotic stabilizer functioning to avoid the destruction of protoplasts exposed ${ }^{15}$, to remove the digested cell wall debris and other contaminants, by gently applying and sucking up the solution with pipette several times. This step was followed by additional drying in a vacuum chamber $(\sim$ 50 Torr) prior to matrix application. In parallel, another vibratome section was also prepared as a control by undergoing the same steps except for the enzymatic digestion.

An oscillating spray device, that was modified from a commercial airbrush (Aztek A470; Testor; Rockford, IL) by replacing the inner spraying tip with a fused silica capillary $(100 \mu \mathrm{m}$ i.d., $360 \mu$ m o.d.; Polymicro Technologyl Phoenix, AZ), was used to homogenously apply colloidal silver. Matrix was transferred to the modified airbrush through a syringe pump (Fisher Scientific; Pittsburgh, PA) and sprayed onto the enzymatically treated cross-section by nebulizing with $\mathrm{N}_{2}$ gas at a pressure of 40 psi. The distance between the tip of the capillary and the sample plate was fixed at $9 \mathrm{~cm}$. The matrix flow rate was set at $50 \mu \mathrm{L} \mathrm{min}^{-1}$ and total volumes sprayed were $0.15 \mathrm{~mL}$. The matrix was applied by spraying for $12 \mathrm{~s}$ duration, suspended by $30 \mathrm{~s}$ intervals to allow for vaporization of the solvent and thus reducing aggregation of the matrix droplets.

Mass Spectrometry Imaging. A high-resolution mass spectrometer with a MALDI source (MALDI LTQ-Orbitrap Discovery; Thermo Electron) was used for profiling and imaging ${ }^{16}$. It has a mass resolution of 30,000 at $\mathrm{m} / \mathrm{z} 400$ and a mass accuracy of $5 \mathrm{ppm}$ with external calibration and 3 ppm with internal calibration. The $\mathrm{N}_{2}$ laser (337 nm, MNL 100; Lasertechnik Berlin; Berlin, Germany) with a maximum energy of $80 \mu \mathrm{J} /$ pulse and maximum repetition rate of 
$60 \mathrm{~Hz}$ is equipped for the system and directly attached to the mass spectrometer. The laser beam from the source is transferred through mirrors and lenses. A black-painted stainless steel plate with a pinhole aperture with a $3 \mathrm{~mm}$ diameter was utilized to adjust the laser spot size to an achieved laser spot size of $\sim 25 \mu \mathrm{m}$. The MALDI source was maintained at the pressure of 75-80 mTorr.

Data Acquisition and Processing for Mass Spectrometric Imaging. Data were acquired from exposed leaf protoplasts prepared by incubation with enzyme mixture followed by matrix application with a fixed number of laser shots without use of automatic gain control (AGC). The number of laser shots and laser power were optimized based on tests conducted on a small area of a sample tissue with AGC turned-on. The sections were scanned with a step size of $35 \mu \mathrm{m}$ for the data acquisition for MS imaging. The MS imaging data were obtained over a $m / z$ 150-1000 range in the positive ion mode. Mass spectrometric images were generated with a tissue imaging visualization software (ImageQuest 1.0.1; Thermo Finnigan). Ion signals of interest were normalized with respect to either total ion current (TIC) on each mass spectrum and the mass window of $\pm 0.008 \mathrm{Da}$ was used to construct the MS images.

\section{Results and Discussion}

Figure 2 shows the SEM images visualizing structural changes of vibratome sections from the Arabidopsis leaf caused by its enzymatic treatment for $20 \mathrm{~min}$ at $37^{\circ} \mathrm{C}$. The incubation time was optimized to 20 min based on the preliminary observations by bright-field microscopy on the vibratome sections at various times with respect to minimal tissue disintegration. As can be seen in Figure 2A-D, a clear difference is observed in the outer surface of the cross sections between the enzymatically-treated and untreated sections. The removal of the cell walls is also shown in 
the higher magnification image of the digested section in Figure 2D compared to that of undigested section (Figure 2C) showing the presence of cell walls. In the SEM image of the enzymatically treated section (Figure 2D), chloroplasts are visualized through the plasma membranes of the protoplasts. Therefore, the removal of cell walls was empirically confirmed by SEM images.

\section{In Situ MS Profiling of Metabolites in the Exposed Cells after Enzymatic Treatment}

of Leaf Vibratome Sections. After the enzymatic digestion of cell walls of internal cells, these cells were subjected to matrix application and MS imaging by two successive laser scans on the same lateral position with one laser shot per scan throughout all data points of the sections imaged. As a control, the vibratome section untreated with enzyme was also subjected to MS imaging and the data from two different samples are compared with each other.

From MS profiling on the enzymatically digested vs. undigested vibratome sections, while there is a large difference in the spectral features between the first and second scan acquired on the same data point of the enzymatically treated vibratome section (Figure 3A), the two mass spectra from the successive scans on the untreated vibratome section do not show much difference except for the distinct appearance of the peak at $\mathrm{m} / \mathrm{z} 573$ on the second scan (Figure 3B). For the enzymatically treated section, many peaks newly detected in a $m / z 420-640$ range on the second scan could be explained by the assumption that our enzymatic treatment of the vibratome sections exposing the internal cells induced the effective digestion of cell walls and rendered the internal cells more vulnerable to laser detection than those shielded by cell wall surfaces in the untreated vibratome section. This can be easily understood by the structural changes in the vibratome section caused by the enzymatic treatment shown in the SEM images (Figure 2). For undigested section, the protoplasts are not exposed as those on the enzymatically 
digested section, and hence even the successive laser detection does not allow profiling of any metabolites in the internal cell layers below due to the presence of the cell walls.

Among the peaks detected on the second scan from the enzymatically treated section, which presumably arise from the metabolites in the internal cell layers, the one detected at $\mathrm{m} / \mathrm{z}$ 385.1286 could be assigned to the silver ion adduct of linolenic acid (C18:3 FA; $\left.\left[\mathrm{C}_{18} \mathrm{H}_{30} \mathrm{O}_{2}{ }^{107} \mathrm{Ag}\right]^{+}\right)$through the chemical composition analysis with a mass accuracy of $-1.3 \mathrm{ppm}$ and its spatial distribution was revealed on the MS image in comparison with the untreated section. Although not shown clearly on the mass spectrum in Figure 3A, the peak at $m / z 387.145$ was also observed in the zoomed-in mass spectrum and this peak is assigned to the silver ion adduct of linoleic acid $\left(\mathrm{C} 18: 2 \mathrm{FA} ;\left[\mathrm{C}_{18} \mathrm{H}_{32} \mathrm{O}_{2}{ }^{107} \mathrm{Ag}\right]^{+}\right)$with a mass accuracy of $-2.2 \mathrm{ppm}$.

Similarly, the peak at $m / z 573.4160$ on the second scan on the undigested vibratome section could be assigned to the silver ion adduct of $\mathrm{C} 32$ alcohol $\left(\left[\mathrm{C}_{32} \mathrm{H}_{66} \mathrm{O}^{107} \mathrm{Ag}\right]^{+}\right)$with a mass accuracy of $0.1 \mathrm{ppm}$ and its localization was also unveiled in the MS images. A pair of silver ion adducts arising from the isotope $\left({ }^{109} \mathrm{Ag}\right)$ are also seen on the mass spectrum for all three species. It is noteworthy to mention that a group of peaks appearing on the second scan from the enzymatically treated section is not attributed to the components or contaminants in enzyme digestion medium applied to the vibratome section because there is almost no difference in the spectral feature of the mass spectra of the first scan for both enzymatically treated and untreated sections.

Based on the results mentioned above, it was verified that it is possible to use our enzymatic digestion method for in situ profiling and imaging of at least some internal metabolites.

\section{In Situ MS Imaging of Metabolites in the Internal Cell Protoplasts after Enzymatic}

Treatment of Leaf Vibratome Section. Figure 4 shows the MS images of silver ion adducts of 
linolenic acid (C18:3 FA) ( $\mathrm{m} / \mathrm{z}$ 385.129), linoleic acid (C18:2 FA), and C32 alcohol $(\mathrm{m} / \mathrm{z} 573.416)$ normalized to the total ion current (TIC). The peaks assigned to linolenic acid (C18:3 FA) $(\mathrm{m} / \mathrm{z}$ 385.129) and linoleic acid (C18:2 FA) $(\mathrm{m} / \mathrm{z}$ 387.145) are the characteristic peaks distinctively appearing after a first laser shot on the vibratome section treated with enzyme. While linolenic acid (C18:3 FA) is spatially distributed overall at high abundance on the section digested with enzyme, it is barely detected on the undigested section and its abundance is much lower than digested section. It should be noted that the sectional region below the white dotted line is damaged during the sample preparation, and therefore is not taken into account to examine the spatial distribution of the metabolites of our interest. The detection of linolenic acid (C18:3 FA), liberated from the plant cell membranes ${ }^{17,18}$, on the enzymatically-treated vibratome section presumably suggests that protecting cell walls exposed to the surface after sectioning are removed by enzymatic digestion and the cell membranes are exposed which can be sampled by laser in LDI-MS.

Free linolenic acid (C18:3 FA) released from the lipid membrane of the plant cell ${ }^{17,18}$ is known as the precursor for the synthesis of jasmonic acid which plays an important role in selfdefense of the plant by regulating the plant responses against biotic, abiotic stresses including insects, pathogenic attack or wounding by herbivores and so on ${ }^{17,19,20}$. From the previous research, it has been reported that the intracellular level of linolenic (C18:3 FA) and linoleic (C18:2 FA) acids in tomato (Lycopersicon esculentum) leaves increased in response to wounding caused by mechanical damage ${ }^{18}$. Also, there has been a previous report that the level of linolenic acid (C18:3 FA) increased in cultured cells of several plant species that are treated with fungal elicitors $^{21}$. Based on these reports, the higher abundance of linolenic acid (C18:3 FA) on the digested section might indicate a detection of this compound accumulating in the cell resulting 
from a release from membranes as a response to the external stimulus such as mechanical sectioning and/or enzymatic treatment by LDI-MS. However, nothing can be concluded with respect to the relations between the high abundance of linolenic acid (C18:3 FA) on the digested section and the effect of two combinational experimental treatments on the leaf tissue only from this work. From MS imaging, jasmonic acid was not detected on the enzymatically digested section.

Similarly, linoleic acid (C18:2 FA) is also detected at much higher abundance on the enzymatically treated section while it is barely seen on the untreated section, and its distribution is seen along the longitudinal section of the leaf. However, the accumulation level of linoleic acid (C18:2 FA) is lower than that of linolenic acid (C18:3 FA).

In contrast, one of the surface metabolites, $\mathrm{C} 32$ alcohol, is not detected on the enzymatically digested section, but observed in high abundance on the overall parts of the undigested section. Considering the fact that our step size of $35 \mu \mathrm{m}$ does not enable the fine localization of detected molecules on the thin longitudinal section having a thickness of only about $\sim 80 \mu \mathrm{m}$, it can be deduced that the distribution of $\mathrm{C} 32$ alcohol appeared on this MS image mainly comes from the outermost surface cuticular wax layer in the section. Similarly, we also have observed the distribution of the well-known cuticular surface lipid compounds (C29, C31, C33 alkanes at $\mathrm{m} / \mathrm{z}$ 515, 543, 571, respectively; Figure 5A, and C26, C28, C30 fatty acids at $m / z 503,531,559$, respectively and C29 ketone at $\mathrm{m} / \mathrm{z} 529$; Figure $5 \mathrm{~B}$ ) only on the undigested vibratome section, but none of them was found on the digested section. Although they are commonly detected from the undigested section, the spatial distribution and the abundance level of each cuticular wax compound is different from the others. 
From our experimental results, we have seen the plausibility of our method of enzymatic digestion combined with vibratome sectioning as an effective way to expose the internal cells surrounded by cell walls below the cuticle layer and reveal the underlying internal cell layers after subsequent digestion of cell walls. This method allows the in situ profiling and imaging of any internal metabolites with LDI-MS. It is worthwhile to adopt several other matrixes to assist the ionization of various other metabolites which may exist in the internal cell layers revealed by enzymatic digestion; ones that were not be able to be detected with colloidal silver as a matrix.

\section{Conclusions}

The plausibility of in situ profiling and imaging of internal metabolites by MS was demonstrated in this study by combining vibratome sectioning to get around having to remove the surface cuticule layer. The enzymatic digestion method subsequently applied to the vibratome sections exposed the internal cell layers for laser detection. The great potential of our method has been shown by successfully detecting and mapping the distribution of linolenic acid (C18:3 FA) and linoleic acid (C18:2 FA) after cell wall enzymatic treatment. While their distributions are shown along the digested section at high abundance, they are barely detected from the undigested section. In contrast, C32 alcohol, one of the surface cuticular wax compounds, assigned to the characteristic peak arising after first laser shot on the undigested section is found at high abundance, but its accumulation is barely seen on the digested section. Moreover, other representative surface metabolites such as C29-33 alkane, C26-30 FA, and C29 ketone are all detected on the undigested section with different spatial distributions, but none of them was shown on the digested section as expected based on the structural changes caused by enzymatic digestion of the vibratome section shown in our SEM images. MS imaging with other 
types of matrixes may also allow the detection of other internal metabolites in future studies. Also, our method can also give the information on the localization of internal metabolites at the (sub) cellular level if combined with higher spatial resolution by the reduction of the laser spot size down to the submicron level. In the near future, the enzymatic treatment of the intact leaf tissues will be performed with the addition of cutinase to the enzyme cocktail used in this work to simultaneously digest the surface cutin layer as well as the cell walls underneath so that the internal cell layers can be exposed to the surface, which allows the in situ profiling and imaging of internal metabolites even without prior vibratome sectioning adopted in the present work due to the difficulty in obtaining the cutinase enzyme. Preparation of purified cutinase and its application are planned to make our method more direct and convenient.

\section{Acknowledgement}

Acknowledgements are made to Randall L. Den Adel and Tracy M. Pepper in the Microscopy and NanoImaging Factility for their great help and advice on the biological issues confronted in this work. The Ames Laboratory is operated for the U.S. Department of Energy by Iowa State University under Contract No. DE-AC02-07CH11358. This work was supported by U.S. Department of Energy, Office of Basic Energy Science, Division of Chemical Sciences. 


\section{References}

1. Cosgrove, D., Nature Reviews Molecular Cell Biology 2005, 6 (11), 850-861.

2. Northcot.DH, Annual Review of Plant Physiology 1972, 23, 113-\&.

3. Vogg, G.; Fischer, S.; Leide, J.; Emmanuel, E.; Jetter, R.; Levy, A. A.; Riederer, M., J Exp Bot 2004, 55 (401), 1401-10.

4. Kolattukudy, P., Annual Review of Plant Physiology and Plant Molecular Biology 1981, $32,539-567$.

5. Cha, S.; Zhang, H.; Ilarslan, H.; Wurtele, E.; Brachova, L.; Nikolau, B.; Yeung, E., Plant Journal 2008, 55 (2), 348-360.

6. $\quad$ Carvalho, C. M.; Aires-Barros, M. R.; Cabral, J. M., Biotechnol Bioeng 1999, 66 (1), 1734.

7. Wen, M.; Au, J.; Gniwotta, F.; Jetter, R., Phytochemistry 2006, 67 (22), 2494-2502.

8. Jun, J.; Song, Z.; Liu, Z.; Nikolau, B.; Yeung, E.; Lee, Y., Analytical Chemistry 2010, 82 (8), 3255-3265.

9. Heeren, R. M. A.; McDonnell, L. A.; Amstalden, E.; Luxembourg, S. L.; Altelaar, A. F. M.; Piersma, S. R., Applied Surface Science 2006, 252 (19), 6827-6835.

10. Marty, F., Plant Cell 1999, 11 (4), 587-599.

11. Electrophoresis., S. Protoplast Preparation - Recipes.

http://www.serva.de/servaWeb/www_root/documents/Protoplast $\% 20$ Preparation_1.pdf (accessed 03.31).

12. Cha, S.; Song, Z.; Nikolau, B. J.; Yeung, E. S., Anal. Chem. 2009, 81 (8), 2991-3000.

13. Huber, S.; Edwards, G., Physiologia Plantarum 1975, 35 (3), 203-209.

14. Edwards, G.; Robinson, S.; Tyler, N.; Walker, D., Plant Physiology 1978, 62 (2), $313-$ 319.

15. Murai, M.; Yoshida, S., Plant and Cell Physiology 1998, 39 (1), 97-105.

16. Strupat, K.; Kovtoun, V.; Bui, H.; Viner, R.; Stafford, G.; Horning, S., Journal of the American Society For Mass Spectrometry 2009, 20 (8), 1451-1463.

17. Blechert, S.; Brodschelm, W.; Hölder, S.; Kammerer, L.; Kutchan, T. M.; Mueller, M. J.; Xia, Z. Q.; Zenk, M. H., Proc Natl Acad Sci U S A 1995, 92 (10), 4099-105. 
18. Conconi, A.; Miquel, M.; Browse, J. A.; Ryan, C. A., Plant Physiol 1996, 111 (3), $797-$ 803.

19. Delker, C.; Stenzel, I.; Hause, B.; Miersch, O.; Feussner, I.; Wasternack, C., Plant Biol (Stuttg) 2006, 8 (3), 297-306.

20. Bonaventure, G.; Baldwin, I. T., Plant Signal Behav 2010, 5 (3), 287-9.

21. Gundlach, H.; Müller, M. J.; Kutchan, T. M.; Zenk, M. H., Proc Natl Acad Sci U S A 1992, 89 (6), 2389-93. 


\section{Figure Captions}

Figure 1. Simplified schematic diagram of a primary plant cell wall and cuticle. Adapted from

(1) Molecular Cell Biology, $4^{\text {th }}$ edition. Lodish H., Berk A., Zipursky SL., et al., New York:

W.H.Freeman, 2000;

(2) Cuticular wax formation by epidermal cells, Samuels, L.; Kunst, L.; Jetter, R., Sealing plant surfaces, Annu. Rev. Plant Biol. 2008, 59, 683-707;

(3) Self-healing of voids in the wax coating on plant surfaces, Koch, K.; Bhushan, B.; Ensikat, H.-J.; Barthlott, W., Philosophical Transactions of the Royal Society a-Mathematical Physical and Engineering Sciences. 2009, 367 (1894), 1673-1688;

(4)http://en.wikipedia.org/wiki/Plant_cell_wall.

Figure 2. SEM images showing structural changes (indicated by the dotted ellipse in B and the zoomed-in image, D) of vibratome sections from the Arabidopsis leaf caused by enzymatic treatment for $20 \mathrm{~min}$ at $37^{\circ} \mathrm{C}$. Two bottom images $(\mathrm{C}, \mathrm{D})$ are higher magnification images of the region indicated with the dotted circle on the top images (A, B).

A, C. Vibratome section not subjected to enzymatic treatment, B, D. Vibratome section exposed to enzymatic treatment.

Figure 3. In situ MS profiling of metabolites in the exposed cells after enzymatic treatment of leaf vibratome sections using colloidal silver as a matrix. (* labeled peaks arise from matrix or contamination) 
A. The mass spectrum over a $\mathrm{m} / \mathrm{z}$ 150-1000 range from the successive scans of the same data point on the vibratome section treated with enzyme mixture for $20 \mathrm{~min}$. There are many peaks newly appeared on the second scan and this is presumably because the enzymatic treatment of the prepared section results in the effective digestion of cell walls and renders the internal cells more vulnerable to laser detection than those exposed to the surface in the untreated vibratome section. Among these peaks, the peaks at $\mathrm{m} / \mathrm{z} 385.129, \mathrm{~m} / \mathrm{z} 387.145$ are assigned to the silver ion adduct of linolenic acid (C18:3 FA; $\left.\left[\mathrm{C}_{18} \mathrm{H}_{30} \mathrm{O}_{2}{ }^{107} \mathrm{Ag}\right]^{+}\right)$, linoleic acid (C18:2 FA;

$\left.\left[\mathrm{C}_{18} \mathrm{H}_{32} \mathrm{O}_{2}{ }^{107} \mathrm{Ag}\right]^{+}\right)$with a mass accuracy of $-1.3 \mathrm{ppm},-2.2 \mathrm{ppm}$, respectively through the chemical composition analysis.

B. The mass spectrum over a $m / z$ 150-1000 range from the successive scans of the same data point on the leaf vibratome section not treated with enzyme mixture as a control. Whereas the spectral features of two mass spectra from the successive scans of the same data point are very different for enzymatically treated section, the successive scans do not show much difference except for the detection of the peak at $\mathrm{m} / \mathrm{z} 573.4160$ on the second scan. This peak was assigned to the silver ion adduct of $\mathrm{C} 32$ alcohol $\left(\left[\mathrm{C}_{32} \mathrm{H}_{66} \mathrm{O}^{107} \mathrm{Ag}\right]^{+}\right)$with a mass accuracy of $0.1 \mathrm{ppm}$ through the chemical composition analysis. Although not shown very well in this figure, the zoomed-in spectrum of the second scan also detects various cuticular wax compounds at $\mathrm{m} / \mathrm{z}$ 515.374 (C29 alkane), m/z 543.405 (C31 alkane), m/z 571.437 (C33 alkane), m/z 503.301 (C26 FA), $m / z 531.333$ (C28 FA), $m / z 559.364$ (C30 FA), and $m / z 529.353$ (C29 ketone).

Figure 4. In situ MS imaging of metabolites in the internal cell protoplasts after enzymatic treatment of leaf vibratome section using colloidal silver as a matrix. The MS images of linolenic acid (C18:3 FA), linoleic acid (C18:2 FA), and C32 alcohol are shown. Ion signals of interest 
were normalized with respect to TIC on each mass spectrum. The first two molecules are detected on the digested section at high abundance whereas they are barely detected on the undigested section. The reverse trend was observed for the surface cuticular wax molecule, C32 alcohol as expected from Figure 3B as well as the prior knowledge about this molecule. It should be noted that the sectional region below the white dotted line is damaged during the sample preparation, and therefore is not taken into account to examine the spatial distribution of the metabolites of our interest.

Figure 5. MS images of well-known surface metabolites on the enzymatically digested and undigested vibratome sections, normalized to TIC on each mass spectrum.

\section{A. C29-C33 Alkanes. \\ B. C26-C30 FA and C29 ketone.}

The distribution of theses well-known cuticular surface lipid compounds are detected only on the undigested vibratome section, but none of them was found on the digested section. Although they are commonly detected from the undigested section, their spatial distribution and abundance level are all different. 


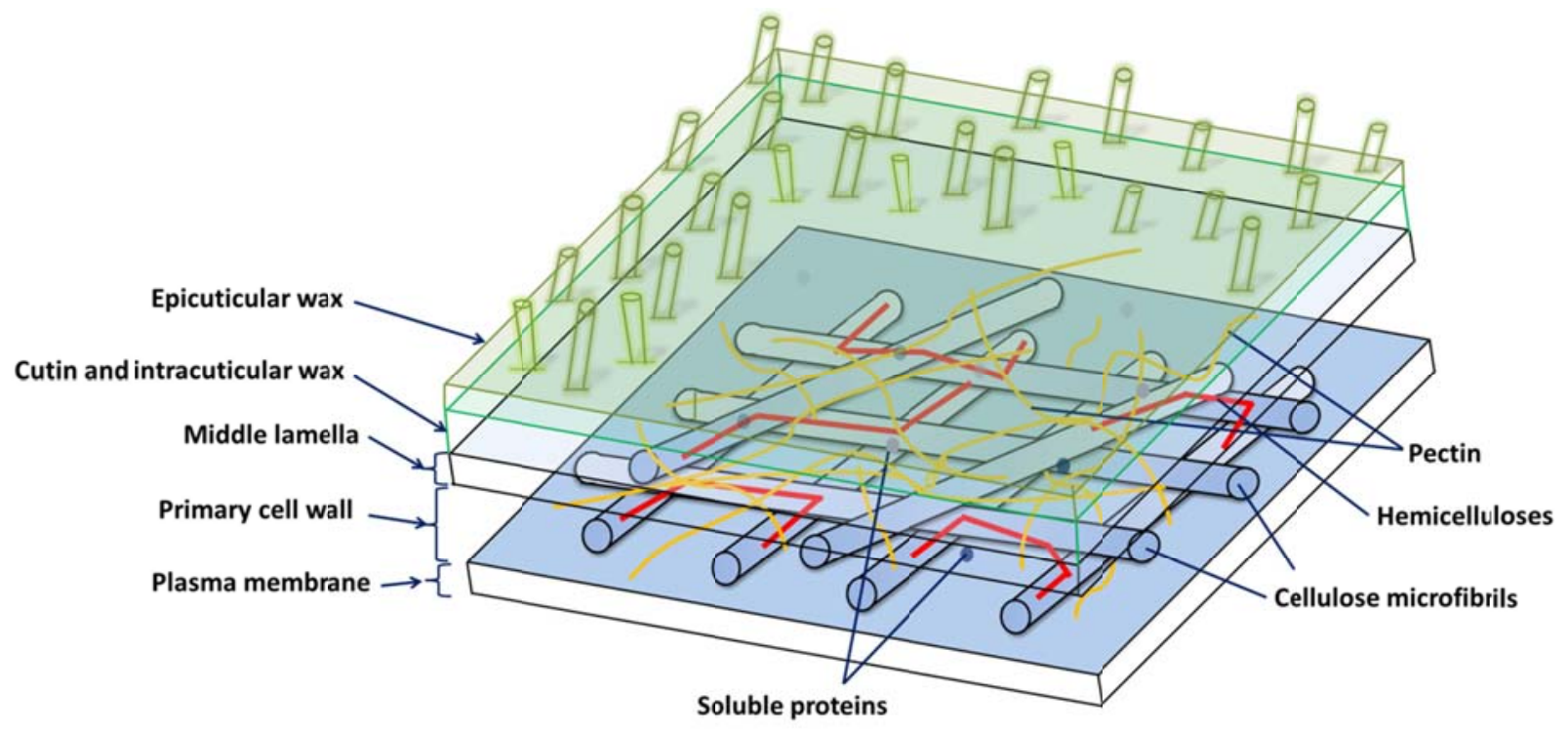

\section{Figure 1.}




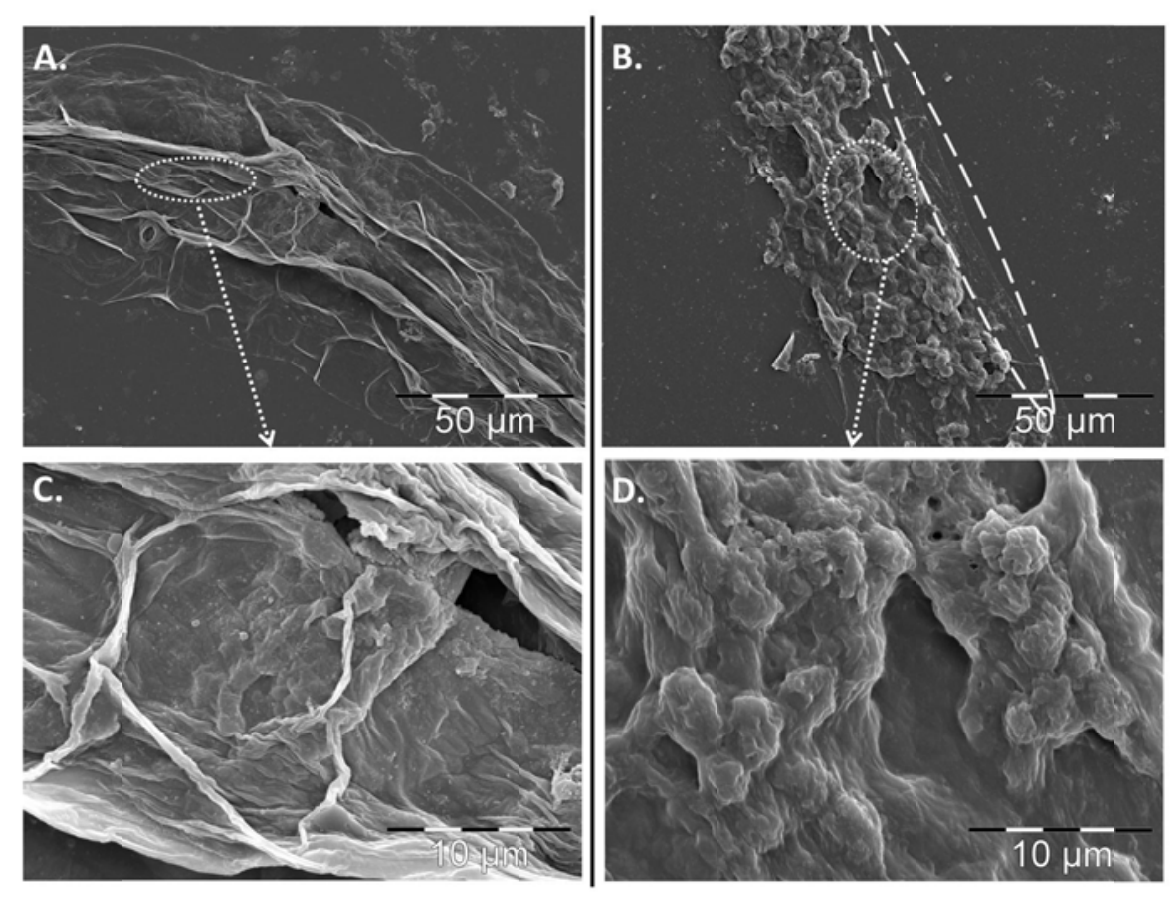

Figure 2. 

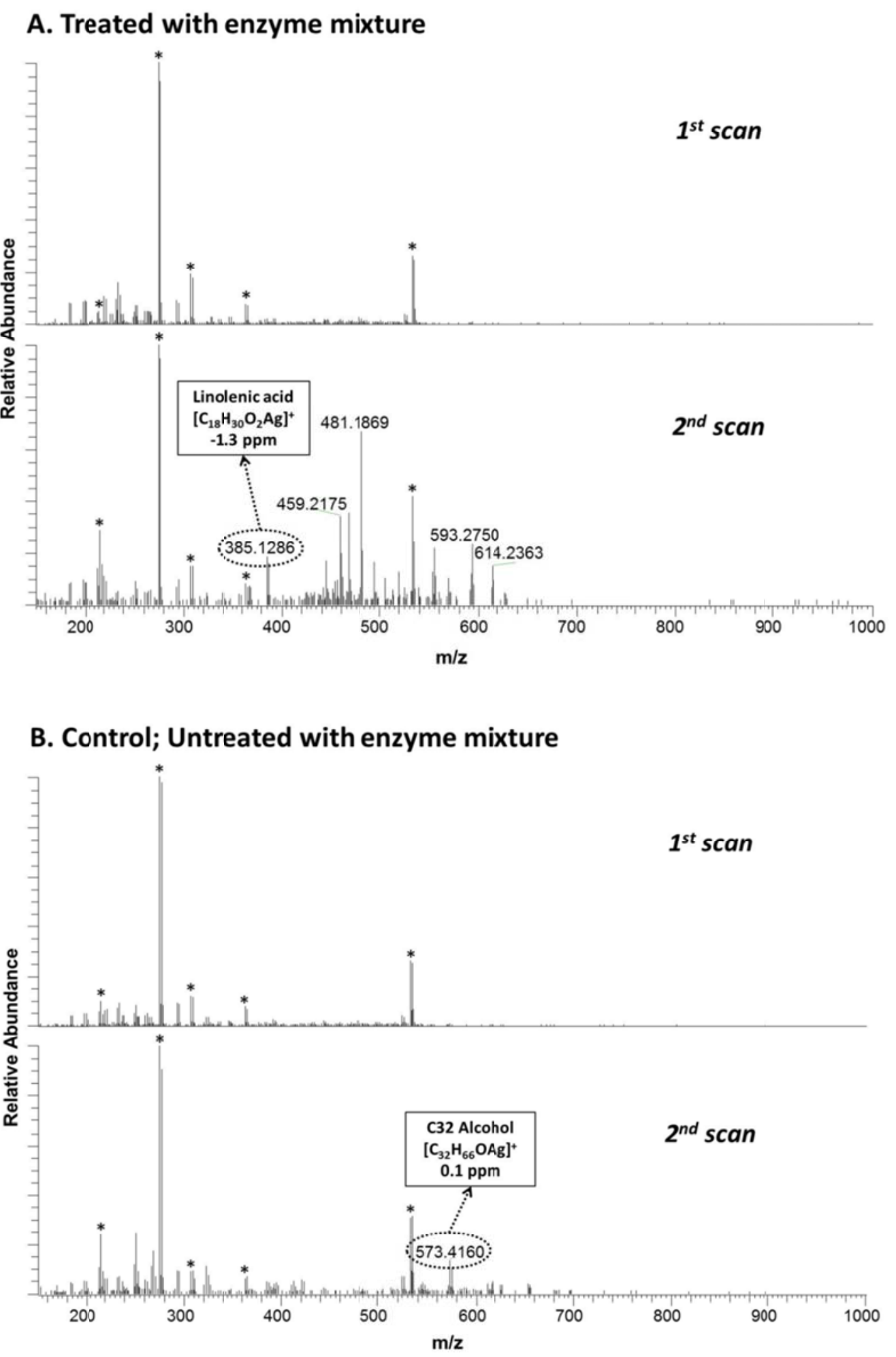

Figure 3. 
- MS imaging with colloidal silver as a matrix

[Mass Range/ TIC]

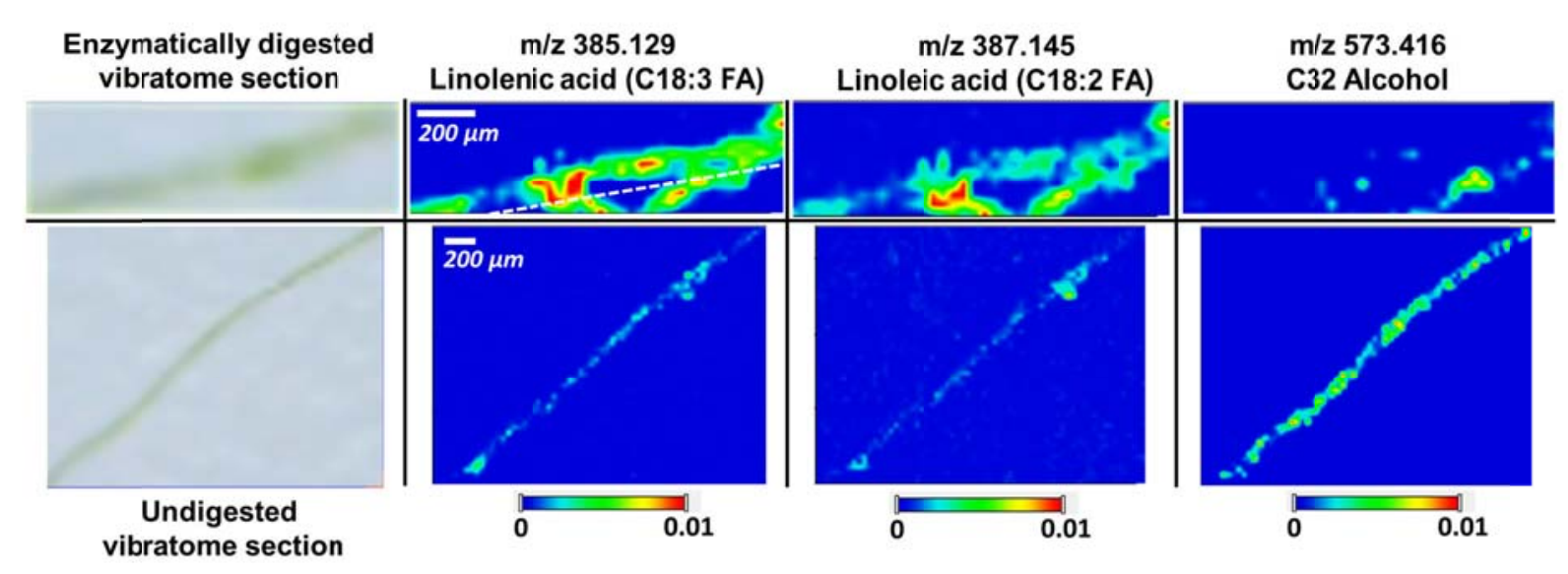

Figure 4. 
- MS imaging with colloidal silver as a matrix

[Mass Range/ TIC]

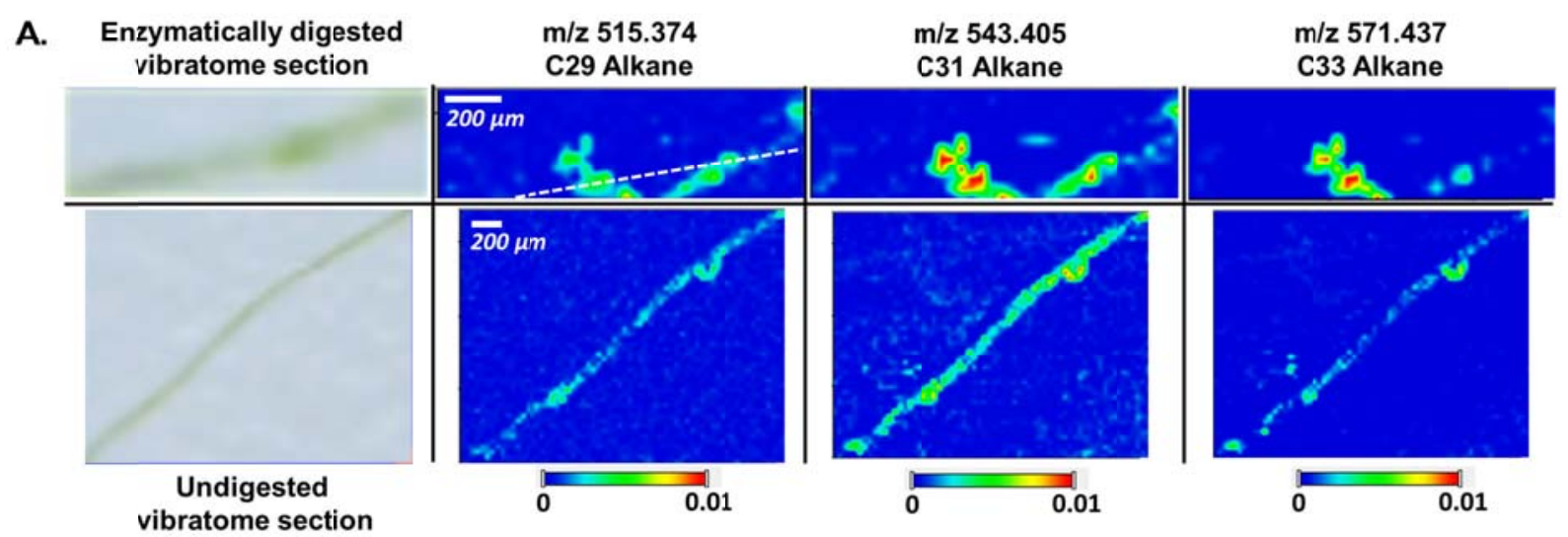

Figure 5. 
- MS imaging with colloidal silver as a matrix

\section{[Mass Range/ TIC]}

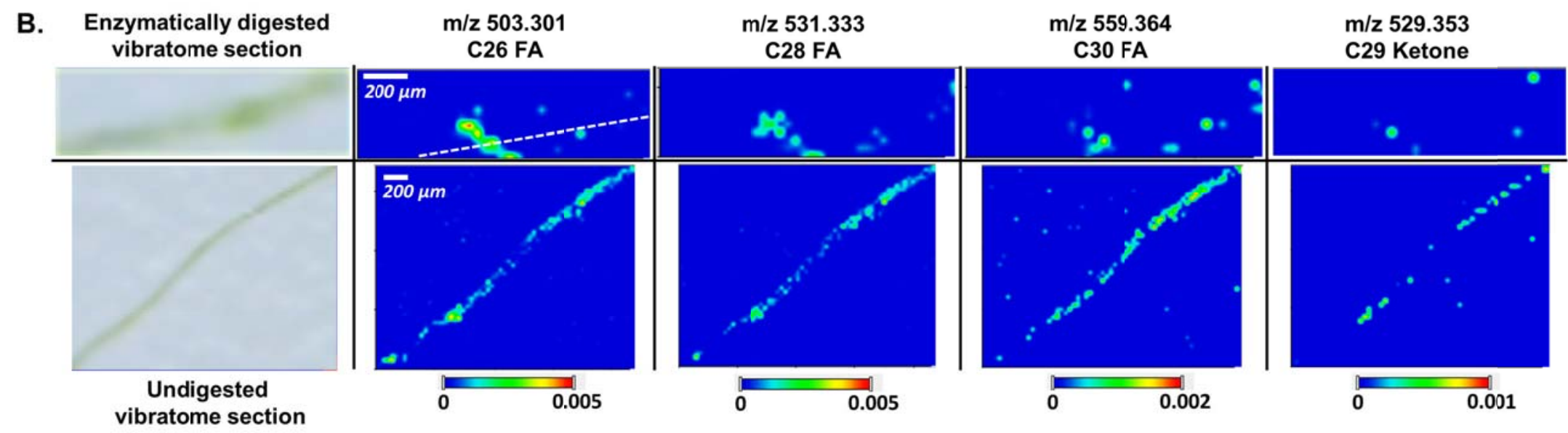

Figure 5. 


\title{
CHAPTER 5. DEVELOPING A MALDI MS IMAGING ASSAY TO LOCALIZE CHLOROPLASTS AND QUANTIFY THE ABUNDANCES OF CHLOROPHYLL $a$ AT THE SINGLE ORGANELLE LEVEL
}

\author{
Manuscript in preparation for submission \\ Ji Hyun Jun, Wei Sun, Basil J. Nikolau and Young Jin Lee
}

\begin{abstract}
An assay for the relative quantification of analytes at the single subcellular/organelle level was developed using mass-assisted laser desorption ionization (MALDI) mass spectrometric (MS) imaging. As a test system, native plant organelle, chloroplast, were directly fractionated from the plant tissues and dispersed at a low density onto the target plate coated with matrix homogeneously through sublimation. The concentration of chloroplast suspension is dilute enough to ensure the detection of an individual organelle at each spot using specific laser spot sizes and laser rastering parameters. The low density of chloroplasts was confirmed first by fluorescence microscopy and the target plate was subsequently subjected to MS imaging. The ion signals of chlorophyll $a(\mathrm{Chl} a$ ) degradation products such as pheophytin $a$ and pheophobide $a$ were monitored to localize the chloroplasts and evaluate the relative abundance of analyte within at the single organelle level. At various densities, the number of chloroplasts and their localization detected in MS image greatly match those detected by fluorescence signal arising from the chlorophyll pigment inside the chloroplasts. A good correlation between the two methods was observed with better correlation at low density. The plausibility of relative
\end{abstract}


quantification is further tested by correlating between ion signal intensity of pheophytin $a$ and pheophobide $a$ and the fluorescence intensity on a pixel-to-pixel basis. The proportional correlation is observed between the signals detected by two methods only for the chloroplasts without significant leakage of chlorophyll within. It is suggested to perform MS imaging without the prior fluorescence measurement to minimize the chloroplast damage. In this preliminary study, we see both plausibility and significant obstacles. Further investigation is necessary to make this method more complete and to develop MALDI MS imaging as an effective analysis tool to evaluate a relative accumulation of analytes of interest at the single organelle level.

\section{Introduction}

The qualitative and quantitative analysis of chemical species in single cells instead of bulk plant tissue provides valuable information on the functional mechanism of plants ${ }^{1,2}$ in that even genetically identical cells under the same environmental conditions exhibit the different phenotypes and varied molecular composition ${ }^{1,3-6}$. These findings as well as importance of differentiating cellular variations automatically led to the need for the development of analytical methods at the single cell level. For this purpose, methods utilizing voltammetry, microcolumn liquid chromatography (LC) ${ }^{7}$, capillary electrophoresis (CE) ${ }^{8}$, mass spectrometry ${ }^{1-2,9-11}$, and fluorescence imaging ${ }^{12}$ have been developed.

Previously, we developed a tool to study single cell level populations of cholesterol in astrocytes using laser desorption ionization (LDI) mass spectrometric (MS) imaging ${ }^{13}$. Here, we extend this approach to subcellular organelles in an attempt to develop an assay using matrixassisted laser desorption ionization (MALDI) MS imaging to localize and quantify analytes at 
the single cell and/or single organelle level. As a probe system, chlorophyll ion signal in single chloroplasts isolated from spinach leaf tissues is monitored to localize the subcellular organelles dispersed at a low density. Fluorescence signal is also monitored in parallel to confirm the MS signal as reflective of the accumulation of analyte molecules within the single organelle.

Isolated chloroplast is a useful target system for our study because it contains the lightharvesting pigments such as $\mathrm{Chl} a, \mathrm{Chl} b$, and catenoids ${ }^{14}$. These compounds yield both MALDI MS signals and fluorescence signal detectable with another analytical method, fluorescence microscopy. The use of fluorescence microscopy as a complimentary detection method is very helpful to confirm the single-organelle-level detection due to the intrinsic fluorescence of chlorophyll within the cell. The qualitative and quantitative analysis of isolated chloroplast in this study may contribute to the understanding of the bulk property of leaf tissues. Furthermore, the plant biology at the macroscopic scale as the properties of leaf tissue is determined by various factors including morphology, anatomy, pigment content and composition, distribution of chloroplasts within the tissue ${ }^{14}$.

There are several advantages of using MALDI MS imaging for the study of biological systems at the single cell and/or single organelle level. First of all, there is no need to tag or preselect the analytes of interest ${ }^{15}$ or have a priori knowledge about the analyte composition. Secondly, it has high sensitivity ${ }^{15}$. Thirdly, the spatial information is preserved within the cells and dilution of biomolecules or contamination, which is caused by the adjacent cells, is reduced ${ }^{15}$ compared to other analytical methods for single cell studies. Lastly, one data acquisition enables the analysis of many organelles spread on the target plate at once. However, the minute amount of analytes within a cell ${ }^{10,15}$ and the small size of the cell make it hard to probe with 
laser ${ }^{9,15}$. These are the main disadvantages of performing the single cell level profiling of biomolecules with MALDI MS.

In the present work, MALDI MS imaging has been utilized as an analytical method to profile and quantify analytes within isolated subcellular organelles. Although the plausibility of this analytical method has been demonstrated, several obstacles were also found, which in turn significantly inhibit the use of MS imaging to visualize and quantify analytes at the single organelle level. Specifically, the instability of native chloroplasts and their tendency to aggregate was a main hurdle to overcome as confirmed by both MS imaging and fluorescence microscopy. Further work is necessary to maintain the integrity, to increase stability of chloroplasts, and to confirm a correlation between the MS signal intensity and the fluorescence intensity, which arise from the analytes within the single organelle representing their accumulation level. After the main limitations are resolved, MALDI MS imaging will be able to become an effective assay for monitoring and quantifying the analytes of interest at the single organelle level, which helps a better understanding of plant biology.

Although many studies have been previously done to perform measurements of biomolecules such as proteins and peptides ${ }^{16-19}$ or cholesterol ${ }^{13}$ in single animal cells using MALDI MS, this is to the best of our knowledge the first work attempting to localize and relatively quantify analytes within the subcellar organelle isolated from the plant tissues.

\section{Experimental Section}

Chemicals. Chloroplast isolation kit containing chloroplast isolation buffer $5 \mathrm{x}$ (CIB), Percoll ${ }^{\circledast}$, bovine serum albumin (BSA), filter mesh, sucrose ( $\left.\geq 99.5 \%\right)$, HEPES buffer $(\geq$ 
$99.5 \%$ ), and ammonium sulfate ( $\geq 99.0 \%$ ) were purchased from Sigma-Aldrich (St. Louis, MO).

2, 5-Dihydroxybenzoic acid (DHB; $99 \%$ ) was obtained from Acros Organics (Morris Plains, NJ).

Isolation of Intact Chloroplasts. Chloroplasts were isolated from $30 \mathrm{~g}$ of spinach leaves purchased from local markets according to the procedure provided for chloroplast isolation kit by Sigma-Aldrich ${ }^{20}$. Briefly, pieces of cut spinach leaves were homogenized with chloroplast isolation buffer using a blender. The homogenate underwent sequential centrifugations at $200 \mathrm{xg}$ to remove unwanted whole cells or cell wall debris. This was followed by another centrifugation at $1,000 \mathrm{x}$ g of supernatant. To purify the intact chloroplast from the broken chloroplast, green chloroplast pellet suspension was layered on a $40 \%$ Percoll $^{\circledR}$ gradient and centrifuged at $1,700 \mathrm{x}$ g. All of the steps described above were completed at $4{ }^{\circ} \mathrm{C}$ and buffers and equipment were precooled prior to the conduction of experimental procedures for chloroplasts isolation. All centrifugations were also performed at 2 to $4{ }^{\circ} \mathrm{C}$ with pre-cooled rotors as recommended by the manual provided by Sigma. Because the isolated chloroplasts are known to lose their activity rapidly, it is recommended to keep the chloroplast suspension in the dark, on ice, until further use. Therefore, the time duration between isolation of chloroplasts and the following experimental steps was minimized to prevent degradation.

Sample Preparation for Fluorescence Microscopy and LDI MS Imaging. After intact chloroplasts were isolated from spinach leaves, chloroplast suspension was diluted with $0.3 \mathrm{M}$ sucrose in $50 \mathrm{mM}$ HEPES buffer $(\mathrm{pH}=7.5)$ at a few different ratios. When the chloroplast suspension is diluted with HEPES buffer, osmolarity of the solutions should be maintained by having sucrose concentration at about $0.3 \mathrm{M}$; otherwise, the isolated chloroplasts are broken ${ }^{21}$. Appropriate chloroplast suspension/buffer ratios were determined prior to MS imaging based on the observation of chloroplasts density in a droplet spotted onto the microscope slide by 
fluorescence microscopy. To overcome the significant aggregation of isolated chloroplasts observed by fluorescence microscopy, $0.1 \mathrm{M}$ ammonium sulfate was added to the diluted aliquot at $1: 2 \mathrm{v} / \mathrm{v}$ ratios.

At the same time, microscope glass slide used as a target plate was prepared by coating with DHB through sublimation method described by Hankin and co-workers ${ }^{22}$. Briefly, $300 \mathrm{mg}$ DHB powder was placed inside the base component of the sublimation apparatus (Chemglass Life Sciences; Vineland, NJ) which is kept at the reduced pressure for 10-15 min. The coolant- dry ice and acetone -was then added on the top part of the apparatus. After $5 \mathrm{~min}$, the sublimation apparatus was gradually heated from 25 to $300{ }^{\circ} \mathrm{C}$ according to the temperature values indicated by the heating plate. After sublimation was completed, which was confirmed by the deposition of a thin matrix layer onto the target plate, the heating source and coolant were removed from the sublimation apparatus. The target plate was allowed to come to room temperature before repressurizing. This method gives highly reproducible, homogeneous coating with a microcrystalline morphology.

After coating of the target slide with DHB matrix, laser burn marks were generated by defining a square area of $1000 \mu \mathrm{m} \times 1000 \mu \mathrm{m}$ on the Tune Plus, software provided by Thermo Finnigan, and firing the boundaries of selected area using high laser power. This step was intended for the detection of chloroplasts distributed within the predefined area by fluorescence microscopy and MS imaging and allow for matching of the experimental results from two methods with ease.

Chloroplast suspension $(0.5 \mu \mathrm{L})$ prepared following the steps described above was spotted within the predefined area marked on the DHB-coated microscope glass slide. Fluorescence 
measurements were performed on the spotted sample first, followed by MS imaging with a minimal time gap between each method.

\section{Imaging Chloroplasts in Fluorescence Microscopy and Differential Interference}

Contrast (DIC) Microscopy. An upright Nikon Eclipse 80i microscope equipped with an XCite 120 (Mississauga, Ontario, Canada) fluorescence excitation light source was used in this study. When the Nikon microscope was operated in epi-fluorescence mode, samples were excited with a $(440 \mathrm{~nm} / 40 \mathrm{~nm})$ bandpass filter (Semrock, Rochester, NY), and the emission fluorescence was collected with a $655 \mathrm{~nm}$ longpass filter (Semorock, Rochester, NY). When the microscope was operated in DIC mode, a set of 2 Nomarski prisms, 2 polarizers and a quarterwave plate were installed. The samples were illuminated through an oil immersion condenser (numerical aperture 1.40) and the optical signals were collected with a proper objective. An Andor iXon ${ }^{\mathrm{EM}}+$ camera $(512 \times 512$ imaging array, $16 \times 16-\mu \mathrm{m}$ pixel size, Belfast, Northern Ireland $)$ was used to record the fluorescence images. ImageJ software developed by National Institutes of Health was used to analyze the images.

Mass Spectrometry Imaging. MS imaging of the chloroplast suspension at various densities was performed with a high-resolution mass spectrometer with a MALDI source (MALDI LTQOrbitrap Discovery; Thermo Electron). The mass analyzer has a mass resolution of 30000 at $\mathrm{m} / \mathrm{z}$ 400 and a mass accuracy of $5 \mathrm{ppm}$ with external calibration and $3 \mathrm{ppm}$ with internal calibration. A nitrogen laser (MNL 100; Lasertechnik Berlin; Berlin, Germany) was used in this system and it has a maximum energy of $80 \mu \mathrm{J} /$ pulse and maximum repetition rate of $60 \mathrm{~Hz}$. The laser beam was transferred through mirrors and lenses instead of an optical fiber. A black-painted stainless steel plate with a pinhole aperture with a diameter of $3 \mathrm{~mm}$ was placed in the laser beam path to reduce the laser spot size to $25 \mu \mathrm{m}$. The MALDI source pressure was kept at 75-80 mTorr. 
Data were acquired from the predefined area where chloroplasts are distributed with a dilute concentration with a fixed number of laser shots without automatic gain control (AGC). The number of laser shots and laser power were determined based on tests conducted on the spotted chloroplast suspension which was not as dilute as the sample used for the actual MS imaging. This increased the probability of detecting the dispersed chloroplasts. The selected area was rastered with a step size of $40 \mu \mathrm{m}$ to minimize the redundant sampling of chloroplasts in two adjacent pixels.

\section{Data Processing for Fluorescence Microscopic Image and Mass Spectrometric Image.}

The width of one pixel in fluorescence microscopic image used to match with the MS image corresponds to $\sim 1.97 \mu \mathrm{m}$. Within the defined area for MS imaging, the $\mathrm{x}, \mathrm{y}$ coordinates of the pixel with the highest MS ion signal intensity corresponding to a chloroplast was used to determine the starting data point for data processing of the fluorescence image. After which, a $500 \times 500$ pixel area from the starting point was extracted from the raw data, the fluorescence signal intensity was integrated over $20 \times 20$ pixels representing the fluorescence signal within the area of dimension of about $40 \mu \mathrm{m} \times 40 \mu \mathrm{m}$ corresponding to the spatial resolution of the MS image. Therefore, the total number of pixels obtained from the fluorescence image after the integration is consistent with that of the MS image.

A tissue imaging visualization software (ImageQuest 1.0.1; Thermo Finnigan) was used to generate the mass spectrometric images. The MS images for the compound of interest were constructed by normalizing the ion signals of interest with respect to the base peak of DHB matrix $\left(m / z 273 ;\left[2\left(\mathrm{M}^{-} \mathrm{H}_{2} \mathrm{O}\right)+\mathrm{H}\right]^{+}\right)$for each spectrum using mass windows of $\pm 0.008 \mathrm{Da}$. Similarly, to compare the ion signal intensity from MS imaging with fluorescence signal intensity on a pixel-to-pixel basis, the ion signal intensity of two $\mathrm{Chl} a$-derived molecules, 
pheophytin $a$ and pheophorbide $a$, were summed and this value was normalized by the base peak of DHB $(\mathrm{m} / \mathrm{z} 273)$ at the discrete pixels where chloroplasts were observed by the two methods.

\section{Results and Discussion}

The intactness of chloroplasts isolated from plant leaves was confirmed by differential interference contrast (DIC) microscopy and fluorescence microscopy (Figure 1). The intact chloroplasts were seen as highly refractive with an outer membrane ${ }^{23}$ under the image obtained with DIC microscopy. The fluorescence image also clearly visualized the fluorescing intact chloroplasts because Chl $a$ of photosystem (PS) II emits most fluorescence as a process of deactivation of the excited pigments after the light absorption ${ }^{24}$. The size of chloroplasts observed in two images is consistent with the known values, $\sim 2-10 \mu \mathrm{m}^{25}$.

Resolution of the Isolated Chloroplasts Aggregation. After confirmation of chloroplasts intactness, we observed that the isolated chloroplasts tend to irregularly aggregate forming big groups of chloroplasts even after dilution of chloroplast suspension with a buffer. The following steps were performed to resolve the problems associated with this aggregation, which significantly inhibited MS imaging at the organelle level. Figure 2A shows the distribution of isolated chloroplasts suspended in a dilute concentration on a microscope slide with the addition of $33.3 \mathrm{mM}$ ammonium sulfate salt at a volumetric suspension-to-salt ratio of 2:1 compared to without the addition of the salt. As is clearly seen from the two fluorescence images, addition of ammonium sulfate salt was helpful to overcome the chloroplasts aggregation problem. This is presumably the result of doubly-charged anions of ammonium sulfate salt effectively maintains a negative charge on the surface of the chloroplast which prevents aggregation through 
electrostatic repulsion. An additional experiment was performed to compare the degree of aggregation of chloroplasts with salts containing anions of different charges. In a $320 \mathrm{x}$ dilution, it was observed that less aggregation occurs with the use of ammonium sulfate, which has doubly-charged anion, than the salts with singly-charged anions such as sodium acetate or sodium chloride (data not shown).

MS Analysis of Isolated Chloroplasts. Figure 3A shows the mass spectrum with a $\mathrm{m} / \mathrm{z}$ range of 580-900 from a single pixel of $40 \mu \mathrm{m} \times 40 \mu \mathrm{m}$ from the MS image of isolated chloroplasts which were $2000 \mathrm{x}$ diluted with sucrose-containing HEPES buffer. Based on the observation of the density of chloroplasts dispersed with fluorescence microscopy to determine the dilution factor, the occupancy rate of chloroplasts was only about $\sim 2.2 \%$ at $2000 \mathrm{x}$ dilution considering the ratio of number of chloroplasts observed to the number of pixels $(25 \times 25$ pixels $)$ scanned. This allows MS imaging at the single organelle level. The Chl $a$ from isolated chloroplasts were mainly detected as degradation products over a $\mathrm{m} / \mathrm{z} 580-1000$ range. The intact Chl $a$ was not detected in the mass spectrum of isolated chloroplasts obtained with DHB as a matrix in positive ion mode. As shown in Figure 3A, pheophytin $a$, one of the degradation products lacking a central $\mathrm{Mg}^{2+}$ ion from $\mathrm{Chl} a$ which is the major pigment in chloroplast, and pheophorbide $a$ are detected at $m / z 871.5707\left(\left[\mathrm{M}-\mathrm{Mg}^{2+}+3 \mathrm{H}^{+}\right]^{+} ; \mathrm{M}=\mathrm{Chl} a\right)$ and $m / z 593.2733$ ([pheophytin $\left.a-\mathrm{C}_{20} \mathrm{H}_{38}\right]^{+}$) within a mass error of $5 \mathrm{ppm}$ from theoretical values, respectively. The molecular structure of three compounds mentioned above is shown in Figure 3B. The mass difference of these two degradation products from Chl $a$ by 278 Da comes from the loss of phytyl group $\left(-\mathrm{C}_{20} \mathrm{H}_{38}\right)$. Although the mass spectrum in Figure 3A is taken from a single pixel, ion counts for both degradation products of Chl $a$ - pheophytin $a$, pheophobide $a$ were $9.31 \mathrm{e} 5$ and $1.04 \mathrm{e} 6$, respectively. 
Although expected, the MS signal was barely detected for lipids from the membrane of chloroplasts in our conditions. This may be related to the previous report that the concentration of chloroplast lipids from spinach leaves such as monogalactosyl-diglyceride, digalactosyldiglyceride, and phosphatidylglycerol decreases at $25^{\circ} \mathrm{C}$ before the beginning of chlorophyll degradation ${ }^{26,27}$. This decrease is assumed to occur because of the increasing amount of oxidative degradation of lipids (peroxidation) ${ }^{26,27}$. Our detection of the degradation products from Chl $a$ but not any lipid species in the mass spectrum is understandable based on this report. Although the chloroplasts suspension was kept on ice after chloroplasts fractionation from the leaf tissues, the fluorescence measurement and subsequent MS imaging were both performed at ambient temperature. This may accelerate the degradation of the lipid of the chloroplasts or chlorophyll.

Figure S-1 (in Appendix 3) provides the SEM image of sublimated DHB showing the matrix homogeneity of $\sim 20 \mu \mathrm{m}$, and this is certainly homogenous enough to perform MS imaging at a spatial resolution of $40 \mu \mathrm{m}$. The homogeneous DHB layer gives a MS image of $m / z 273$ with a small variation in intensity. The symmetric Gaussian distribution of the number of pixels with respect to MS intensity shown in the histogram also support that the DHB applied through sublimation is highly homogeneous. It is worth noting that the MS signal of Chl $a$-derived compounds was not detected if the sublimation of DHB is performed on top of the chloroplasts suspension spotted onto the target plate. The thick coating of DHB suppresses the detection of analytes in the chloroplast of our interest even with a high number of laser shots and laser power. Therefore, the sample was spotted onto the DHB coating on the target slide.

MS Imaging of Isolated Chloroplasts. The isolated chloroplasts suspension was $200 \mathrm{x}$, $1000 \mathrm{x}$, and $2000 \mathrm{x}$ diluted with $0.3 \mathrm{M}$ sucrose-containing buffer media and ammonium salt was 
added to these diluted solution at a ratio of 2:1 (diluted solution:salt, $\mathrm{v} / \mathrm{v}$ ). $0.5 \mu \mathrm{L}$ aliquots of the final solutions were spotted within the area of $1000 \mu \mathrm{m} \times 1000 \mu \mathrm{m}$ predefined with high power laser on the MALDI target plate that was pre-coated with DHB by sublimation. Fluorescence images and the MS images performed onto the spotted samples at different density are shown in Figure 4. It should be noted that fluorescence measurement was performed prior to MS imaging considering the general amount of time measurements take with each method. MS imaging usually takes a much longer time than the acquisition of a fluorescence image, the fluorescence of chlorophyll in chloroplast is most likely quenched by the time fluorescence measurement is performed after MS imaging. For example, whereas it took about $\sim 24$ min to acquire the MS image of $1000 \mu \mathrm{m} \times 1000 \mu \mathrm{m}$ with a raster size of $40 \mu \mathrm{m}$, fluorescence measurement did not take longer than $5 \mathrm{~min}$. More importantly, MALDI by virtue of the laser irradiation is a destructive technique. The intactness of chloroplasts is no longer maintained after MS imaging because the laser is used to sample the analytes. For these reasons, performing the two analytical methods in reverse is impossible. It should be mentioned that, however, the time duration between two methods was still minimized as much as possible, so it is important to optimize all experimental parameters from the preceding experiments in advance.

Signal of one of the degradation products of $\mathrm{Chl} a$, pheophytin $a(\mathrm{~m} / \mathrm{z} 871)$ that was normalized against the base matrix peak $(\mathrm{m} / \mathrm{z} 273)$ was monitored to localize the chloroplasts at the single organelle level. At various densities (200 x, $1000 \mathrm{x}$, and $2000 \mathrm{x}$-dilution), the number of chloroplasts and their localization detected as an ion signal of pheophytin $a$ in the MS images greatly match with those detected by fluorescence microscopy as shown in Figure 4. This good correlation between the images obtained by two methods is shown more obvious at the lowest density $(2000 \mathrm{x})$. In the similar context, the attempt to localize the dispersed chloroplasts was 
also made by constructing the MS image of another degradation product of Chl $a$, pheophobide $a$ $(\mathrm{m} / \mathrm{z} 593)$ which signal is normalized by the base matrix peak $(\mathrm{m} / \mathrm{z} 273)$. The normalization process was conducted to decrease the bias in detection of the signal of analytes, which may arise from the homogeneity of matrix ${ }^{28}$. As shown in Figure 5, the localization of the isolated chloroplasts monitored with ion signal of pheophobide $a(\mathrm{~m} / \mathrm{z} 593)$ is in great agreement with that visualized with ion signal of pheophytin $a(\mathrm{~m} / \mathrm{z} 871)$. Therefore, a good match of two MS images of different degradation products of $\mathrm{Chl} a$ supports the hypothesis that pixels with sufficient signal intensity to be visualized in the MS images represent the localization of isolated chloroplasts dispersed on the target plate at low density, which confirms the plausibility of MS imaging at the single organelle level.

From the images localizing the chloroplasts obtained with two experimental methods, the plausibility of relative quantification was tested by correlating ion signal intensity of pheophytin $a$ and pheophobide $a$ normalized with base matrix peak from MS imaging and the fluorescence intensity on a pixel-to-pixel basis. Figure 6 shows the signal intensity profile constructed along a series of $\mathrm{x}$-coordinate at the fixed y-position, $\mathrm{y}=960 \mu \mathrm{m}$ which is indicated on the visualization software for MS imaging for both the MS image and fluorescence image. Since the best correlation was observed between two images at the lowest density of $2000 \mathrm{x}$, the images acquired with the $2000 \mathrm{x}$ diluted sample were used to compare the signal intensities from pixel to pixel. As shown in Figure 6, the position on the x-axis at which the highest signal is detected in the fluorescence image at $y=960 \mu \mathrm{m}$ is in a good agreement with the point of $\mathrm{x}$-axis where the highest MS signal is detected.

However, it is noteworthy that the background signal seen both in the profile of fluorescence intensity plotted and the fluorescence image of Figure 6 is fairly high. Although great care was 
taken to maintain the intactness of chloroplasts and minimize the breakage of chloroplast membrane during the process of sample preparation, the high background signal observed is presumably attributed to problems associated with damage to the chloroplast membrane by the excitation light. This leads to the leaking of the pigments from the cells. The fluorescence excitation light not only excites the chlorophyll in chloroplast but also results in the simultaneous excitation of DHB which is also an absorbing material. This eventually causes damage to the chloroplast membrane. There has been a previous report that the light illumination leads to the change in the gross morphology of the inner chloroplast membrane system and change in the gross morphology of intact chloroplasts isolated from spinach through the sequence of events ${ }^{29}$. Besides the high background signal detected in the fluorescence image, there are several evidences that support our hypothesis about the damage of chloroplasts membrane and leakage of chlorophyll inside. First of all, tails are clearly observed from the points with the bright fluorescence signal indicative of individual chloroplast dispersed on the plate. Also, these tails are all directed toward the same direction, which is also visualized in the MS image. Therefore, the appearance of tails is evidently not an artifact possibly caused by the experimental conditions used for fluorescence measurement but they arise from the actual leakage of chlorophyll as confirmed by the ion signal of degradation products of Chl $a$ visualized in the MS image. Secondly, the size of each fluorescing chloroplast in the fluorescence image is measured approximately 4-10 times bigger than the known size of chloroplast. As a control experiment, imaging of isolated chloroplasts in a dilute condition was performed with fluorescence microscopy without the use of DHB-coated target slide (Figure S-2 in Appendix 3). The fluorescence images in both Figure 4 and Figure S-2 were not subjected to any additional process to substract background and it is obviously seen that the background signal in Figure 4 where the 
sample is spotted onto the DHB-coated slide is much higher than that in Figure S-2. Also, the fluorescence images obtained with the sample deposited on the slides with no DHB coating are free of appearance of tails that were strikingly seen in Figure 4. The overall size of fluorescing chloroplasts is also consistent with the known size of chloroplast. Therefore, it is concluded from these results that, for quantitative analysis at the single organelle level using MS imaging, conducting of a prior fluorescence measurement is not appropriate.

With being aware of this significant bottleneck in quantitative analysis, correlating ion signal intensity of pheophytin $a$ and pheophobide $a$ normalized with base matrix peak with the fluorescence intensity was still attempted on a pixel-to-pixel basis. Figure 7 shows the plot showing the pixel-to-pixel correlation of signals detected by two different methods, MS imaging and fluorescence microscopy. For quantitative analysis of chlorophyll $a$ from MS imaging, ion signals of pheophytin $a(\mathrm{~m} / \mathrm{z} 871)$ and pheophorbide $a(\mathrm{~m} / \mathrm{z} 593)$ are summed and this summed ion signal intensity is normalized by the base peak arising from the matrix $(\mathrm{m} / \mathrm{z} 273)$ at the discrete pixels (a-j) with sufficient ion signal. The integrated fluorescence intensities over the area corresponding to the pixels on the MS image are plotted as a function of ion signals detected by MS imaging. At position of chloroplasts labeled (a), (b) with almost no appearance of tail from its origin, the integrated fluorescence intensity of the $\mathrm{Chl} a$ seems to be proportional to the MS ion signal. However, the linear correlation could not be constructed between the signals obtained with two different analytical methods for the rest of the detected chloroplasts (c-j). As mentioned above, the significant leakage of chlorophyll hinders the reflection of the actual accumulation of Chl $a$ at the single organelle level by MS imaging as well as fluorescence microscopy. For example, although the MS image shows that the chloroplast at the position (j) has the highest MS ion signal among all chloroplasts seen on the image, the fluorescence signal 
is the highest from the chloroplast at the (d) position. This skewed quantitative information for the chloroplast (d) is affected by the adjacent chloroplast (c) because the Chl $a$ originated from chloroplast (c) also contributes to the increase in the signal of both fluorescence and MS imaging for chloroplast (d) considering the direction of tails. On the contrary, chloroplast located at the position (j) does not have any contributor nearby except for chloroplast (b) which has almost no appearance of tail from its origin, and hence the fluorescence signal for chloroplast at (j) is shown lower than those at (c), (d), (e) even with the highest MS signal of Chl $a$-derived compounds. Further study needs to be done to confirm if a good correlation can be observed between signal intensities indicative of accumulation of analytes by both analytical methods without the prior fluorescence measurement before MS imaging. In this preliminary study, we see both plausibility and significant obstacles for quantitative analysis at the single organelle level.

\section{Conclusions}

The possibility of developing an assay for relative quantification of analytes at the single subcellular/organelle level has been suggested using MALDI MS imaging. Two degradation products of Chl $a$, pheophytin $a$ and pheophobide a, were detected in the mass spectrum acquired on the isolated chloroplast. The localization of isolated chloroplasts detected by MS imaging of these compounds is in good accordance with each other. Also, the number of chloroplasts and their localization revealed by MS imaging exactly match with those determined by fluorescence image especially at low density, which confirms the MS imaging at the single organelle level. However, more investigation is necessary to completely resolve the problems associated with 
intactness and stability of chloroplasts so that the quantitative information is still preserved within the individual chloroplast and well reflected by our analytical technique. For this, there may be a need to attempt fixation of the dispersed chloroplasts and see whether it is helpful to reflect the accumulation of chlorophyll within by maintaining the intactness of chloroplast and minimizing the depletion of fluorescence. Also, if the spatial resolution in MALDI MS imaging can be increased to the submicron level, it will enable imaging of the fine localization of analytes even within a single organelle. This was not achievable in the present work due to the use of a larger laser spot size to fully detect the individual chloroplast in each scan.

\section{Acknowledgement}

Acknowledgement is made to Wei Sun for his technical expertise and a great help to acquire the images with fluorescence, DIC microscopy. The Ames Laboratory is operated for the U.S. Department of Energy by Iowa State University under Contract No. DE-AC02-07CH11358. This work was supported by U.S. Department of Energy, Office of Basic Energy Science, Division of Chemical Sciences.

\section{Appendixes}

The SEM image which shows the homogeneity of sublimated DHB is provided in Appendix 3 along with the MS image for $m / z 273$ arising from DHB matrix $\left.\left(2\left(\mathrm{M}-\mathrm{H}_{2} \mathrm{O}\right)+\mathrm{H}\right]^{+}\right)$and the histogram constructed from this MS image. The fluorescence images of isolated chloroplasts at $1000 \mathrm{x}, 2000 \mathrm{x}$ dilution that are spotted onto the target slide with no DHB coating are also shown. 


\section{References}

1. Amantonico, A.; Oh, J.; Sobek, J.; Heinemann, M.; Zenobi, R., Angewandte ChemieInternational Edition 2008, 47 (29), 5382-5385.

2. Tejedor, M.; Mizuno, H.; Tsuyama, N.; Harada, T.; Masujima, T., Analytical Sciences 2009, 25 (9), 1053-1055.

3. Kaern, M.; Elston, T.; Blake, W.; Collins, J., Nature Reviews Genetics 2005, 6 (6), 451464.

4. $\quad$ Raser, J.; O'Shea, E., Science 2004, 304 (5678), 1811-1814.

5. $\quad$ Rao, C.; Wolf, D.; Arkin, A., Nature 2002, 420 (6912), 231-237.

6. Newman, J.; Ghaemmaghami, S.; Ihmels, J.; Breslow, D.; Noble, M.; DeRisi, J.; Weissman, J., Nature 2006, 441 (7095), 840-846.

7. $\quad$ Ewing, A., Journal of Neuroscience Methods 1993, 48 (3), 215-224.

8. $\quad$ Ewing, A.; Wallingford, R.; Olefirowicz, T., Analytical Chemistry 1989, 61 (4), A292-\&.

9. Masujima, T., Analytical Sciences 2009, 25 (8), 953-960.

10. Fung, E.; Yeung, E., Analytical Chemistry 1998, 70 (15), 3206-3212.

11. Li, L.; Garden, R.; Sweedler, J., Trends in Biotechnology 2000, 18 (4), 151-160.

12. Yeung, E., Analytical Chemistry 1999, 71 (15), 522A-529A.

13. Perdian, D.; Cha, S.; Oh, J.; Sakaguchi, D.; Yeung, E.; Lee, Y., Rapid Communications in Mass Spectrometry 2010, 24 (8), 1147-1154.

14. Merzlyak, M.; Chivkunova, O.; Zhigalova, T.; Naqvi, K., Photosynthesis Research 2009, $102(1), 31-41$.

15. Chughtai, K.; Heeren, R., Chemical Reviews 2010, 110 (5), 3237-3277.

16. Garden, R.; Shippy, S.; Li, L.; Moroz, T.; Sweedler, J., Proceedings of the National Academy of Sciences of the United States of America 1998, 95 (7), 3972-3977.

17. JIMENEZ, C.; VANVEELEN, P.; LI, K.; WILDERING, W.; GERAERTS, W.; TJADEN, U.; VANDERGREEF, J., Journal of Neurochemistry 1994, 62 (1), 404-407.

18. Jimenez, C.; Li, K.; Dreisewerd, K.; Spijker, S.; Kingston, R.; Bateman, R.; Burlingame, A.; Smit, A.; van Minnen, J.; Geraerts, W., Biochemistry 1998, 37 (7), 2070-2076. 
19. Worster, B.; Yeoman, M.; Benjamin, P., European Journal of Neuroscience 1998, 10 (11), 3498-3507.

20. Sigma-Aldrich. Chloroplast isolation kit.

http://www.sigmaaldrich.com/etc/medialib/docs/Sigma/Bulletin/cpisobul.Par.0001.File.tmp/cpis obul.pdf (accessed 2012.03.18).

21. Hongladarom, T.; Honda, S. I., Plant Physiol 1966, 41 (10), 1686-94.

22. Hankin, J. A.; Barkley, R. M.; Murphy, R. C., Journal of the American Society for Mass Spectrometry 2007, 18 (9), 1646-1652.

23. Jensen, R. G.; Bassham, J. A., Proc Natl Acad Sci U S A 1966, 56 (4), 1095-101.

24. Krause, G.; Weis, E., Annual Review of Plant Physiology and Plant Molecular Biology 1991, 42, 313-349.

25. Wikipidia. Chloroplast. http://en.wikipedia.org/wiki/Chloroplast (accessed 2012.03.18).

26. Yamauchi, N.; Iida, S.; Minamide, T.; Iwata, T., Journal of the Japanese Society for Horticultural Science 1986, 55 (3), 355-362.

27. Yamauchi, N.; Watada, A., Journal of the American Society For Horticultural Science 1991, $116(1), 58-62$.

28. Duncan, M. W.; Roder, H.; Hunsucker, S. W., Brief Funct Genomic Proteomic 2008, 7 (5), 355-70.

29. $\quad$ Murakami, S.; Packer, L., J Cell Biol 1970, 47 (2), 332-51. 


\section{Figure Captions}

Figure 1. The images of chloroplasts obtained by differential interference contrast (DIC) microscopy (left) and fluorescence microscopy (right). After the isolation of intact chloroplasts from spinach leaves and the subsequent purification of intact chloroplasts from the broken chloroplasts, the chloroplast suspension was diluted at the appropriate ratios to be detected on the microscope slide in a dilute condition. Both images were acquired in the wet condition. The chloroplasts are observable as fluorescence occurs, as known, in the red region of visible light due to the presence of major pigments, chlorophylls, in chloroplasts. The size of chloroplasts observed in two images is consistent with the known values of $\sim 2-10 \mu \mathrm{m}$.

\section{Figure 2.}

A. The comparison of fluorescence images of native chloroplasts suspended in a dilute condition on the microscope slide without (left) and with (right) addition of $33.3 \mathrm{mM}$ ammonium sulfate. As clearly seen from both images, adding ammonium sulfate helps partially resolve the problems associated with aggregation of isolated chloroplasts, which significantly hinders the MS imaging at the single organelle level.

B. The distribution of chloroplasts in $320 \mathrm{x}$ diluted condition with addition of different types of salts detected by DIC and fluorescence microscopy. The addition of doubly-charged anion containing-salt (ammonium sulfate; $\left.\left(\mathrm{NH}_{4}\right)_{2} \mathrm{SO}_{4}\right)$ is more efficient than the salt having singly charged anion (sodium acetate; $\mathrm{NaCH}_{3} \mathrm{COO}$, sodium chloride; $\mathrm{NaCl}$ (data not shown)) to resolve the isolated chloroplasts aggregation problem. This result is presumably because doubly-charged 
anion of salt effectively helps each chloroplast to maintain negative charge on the surface and repel each other in electrostatic interaction.

\section{Figure 3.}

A. The mass spectrum over a $m / z 580-900$ range from a single pixel of $40 \mu \mathrm{m} \times 40 \mu \mathrm{m}$ from MS imaging of isolated chloroplasts which were $2000 \mathrm{x}$ diluted with sucrose-containing HEPES buffer. For both compounds degraded from Chl $a$-pheophytin $a$ and pheophobide $a$ - of our interest, the sufficient ion signal close to e6 was observed even at the single pixel.

B. The molecular structure of $\mathrm{Chl} a$ and its two degradation products, pheophytin $a$ which lacks a central $\mathrm{Mg}^{2+}$ ion from Chl $a$ and pheophorbide $a$ which loses phytyl group $\left(-\mathrm{C}_{20} \mathrm{H}_{38}\right)$ at $\mathrm{R}_{2}$ position from pheophytin $a$.

Figure 4. The fluorescence images and the corresponding MS images of pheophytin $a(\mathrm{~m} / \mathrm{z}$ 871.573) normalized against the base matrix peak $(\mathrm{m} / \mathrm{z} 273)$, from the isolated chloroplasts. At various densities (200 x, $1000 \mathrm{x}$, and $2000 \mathrm{x}$-dilution), the number of chloroplasts and their localization detected as an ion signal of pheophytin $a$ in the MS images greatly match with those detected by fluorescence microscopy. This good correlation between the images obtained by two methods is shown more obvious at the lowest density (2000 x).

Figure 5. The MS images of pheophytin $a(\mathrm{~m} / \mathrm{z} 871)$ and pheophorbide $a(m / z 593)$. The localization of the isolated chloroplasts monitored with ion signal of pheophobide $a(\mathrm{~m} / \mathrm{z} 593)$ is in a great accordance with that visualized with ion signal of pheophytin $a(\mathrm{~m} / \mathrm{z} 871)$. Therefore, from a good match of two MS images of different degradation products of Chl $a$, it is proven that the pixels with sufficient signal intensity to be visualized in the MS images represent the 
localization of isolated chloroplasts dispersed on the target plate at low density, which confirms the plausibility of MS imaging at the single organelle level.

Figure 6. MS imaging at the single organelle level and the corresponding fluorescence image (left) and the signal intensity profile constructed along a series of $x$-coordinate at the fixed $y$ position, $\mathrm{y}=960 \mu \mathrm{m}$ from each image (right). The position in $\mathrm{x}$-axis at which the highest signal is detected in the fluorescence image at $\mathrm{y}=960 \mu \mathrm{m}$ is in a good agreement with the point of $\mathrm{x}$-axis where the highest MS signal is detected.

Figure 7. A plot which correlates the signals detected by two different methods, MS imaging and fluorescence microscopy, on the pixel-to-pixel basis. For quantitative analysis of $\mathrm{Chl} a$ from MS imaging, ion signals of pheophytin $a(\mathrm{~m} / \mathrm{z} 871)$ and pheophorbide $a(\mathrm{~m} / \mathrm{z} 593)$ are summed and this summed ion signal intensity is normalized by the base peak arising from the matrix $(\mathrm{m} / \mathrm{z}$ 273) at the discrete pixels (a-j) with sufficient ion signal. The integrated fluorescence intensities over the area corresponding to the pixels on the MS image are plotted as a function of ion signals detected by MS imaging. The significant leakage of chlorophyll due to the simultaneous excitation of DHB-coating upon the fluorescence excitation significantly hinders quantitative analysis of Chl $a$ at the single organelle level by MS imaging as well as fluorescence microscopy. 

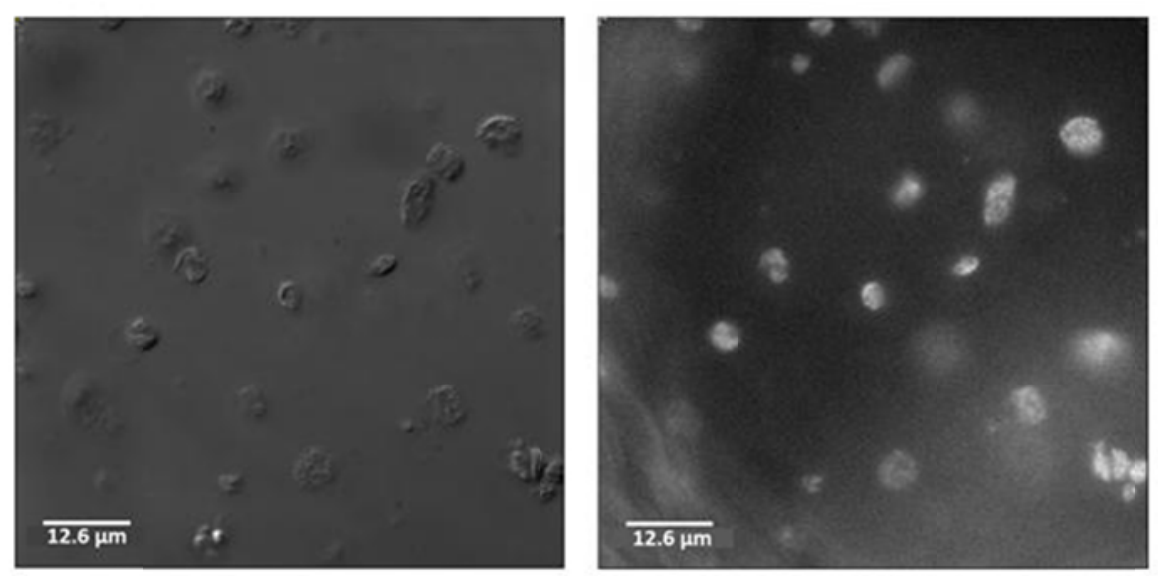

Figure 1. 
A. Without ammonium sulfate With ammonium sulfate

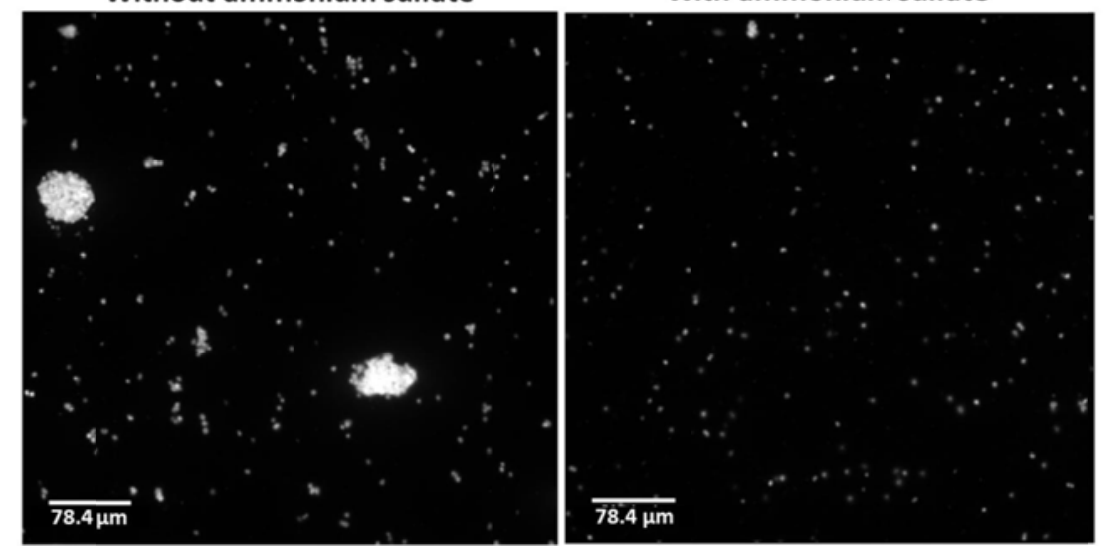

B.

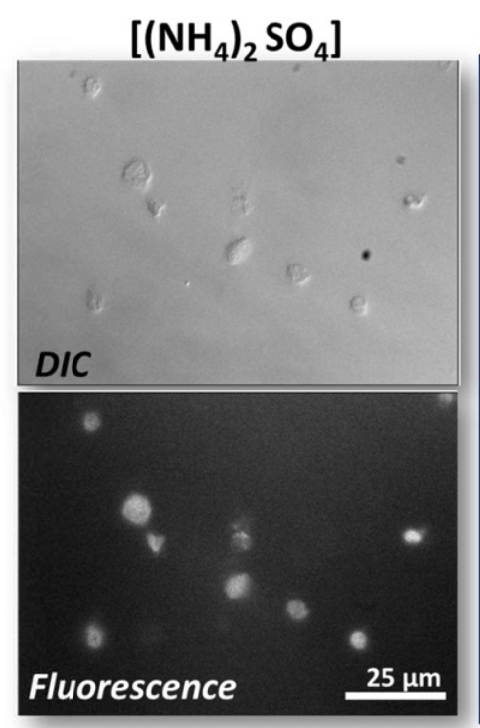

[ $\left.\mathrm{NaCH}_{3} \mathrm{COO}\right]$

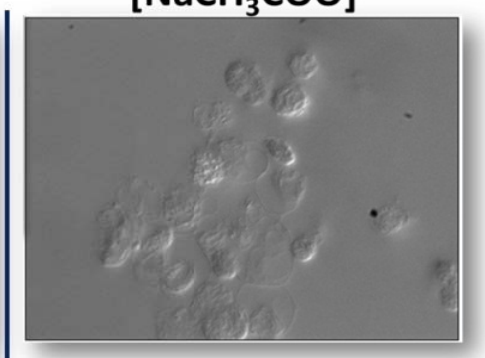

Figure 2. 


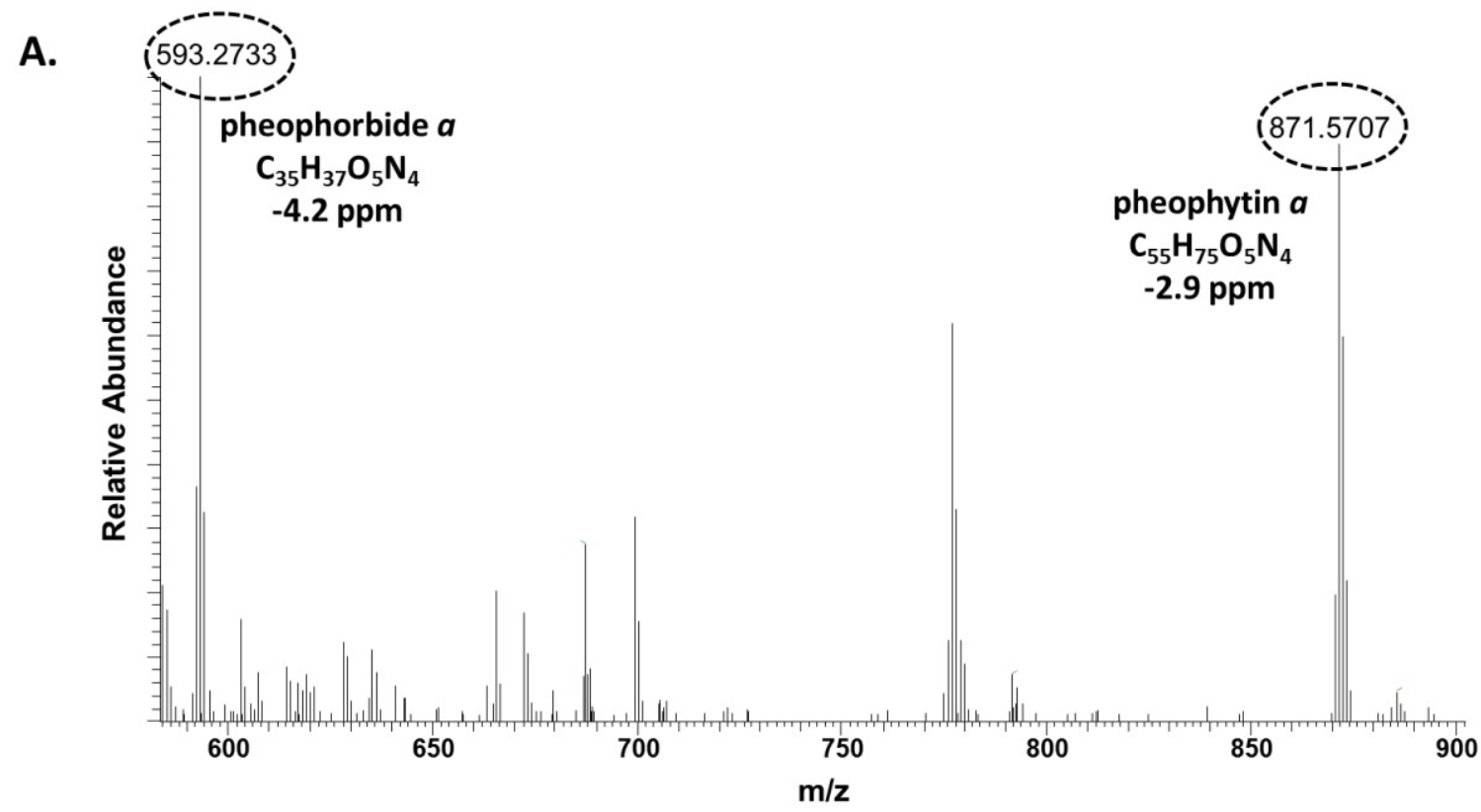

B.

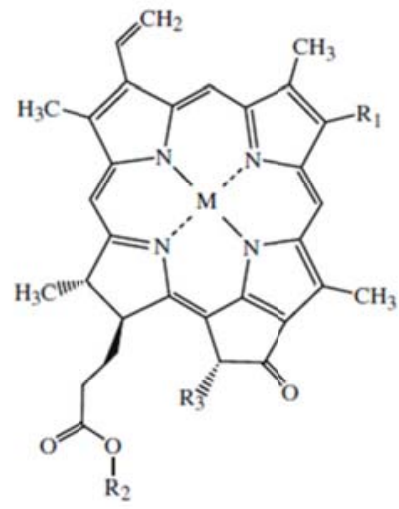

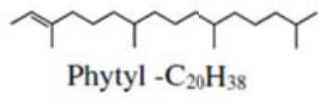

\begin{tabular}{|ccccc|}
\hline Pigment & $\mathbf{M}$ & $\mathbf{R}_{\mathbf{1}}$ & $\mathbf{R}_{\mathbf{2}}$ & $\mathbf{R}_{\mathbf{3}}$ \\
\hline Chlorophyll $a$ & $\mathrm{Mg}$ & $\mathrm{CH}_{2} \mathrm{CH}_{3}$ & Phytyl & $\mathrm{COOCH}_{3}$ \\
\hline Pheophorbide $a$ & $2 \mathrm{H}$ & $\mathrm{CH}_{2} \mathrm{CH}_{3}$ & $\mathrm{H}$ & $\mathrm{COOCH}_{3}$ \\
\hline Pheophytin $a$ & $2 \mathrm{H}$ & $\mathrm{CH}_{2} \mathrm{CH}_{3}$ & Phytyl & $\mathrm{COOCH}_{3}$ \\
\hline
\end{tabular}

\section{Figure 3.}

* Figure for structural formula in B

\footnotetext{
${ }^{*}$ Reprinted from Analytical Biochemistry, 390(1), Suzuki T. et al., Analysis of chlorophylls and their derivatives by matrix-assisted laser desorption/ionization-time-of-flight mass spectrometry, 57-62, Copyright (C) 2009, with permission from Elsevier.
} 


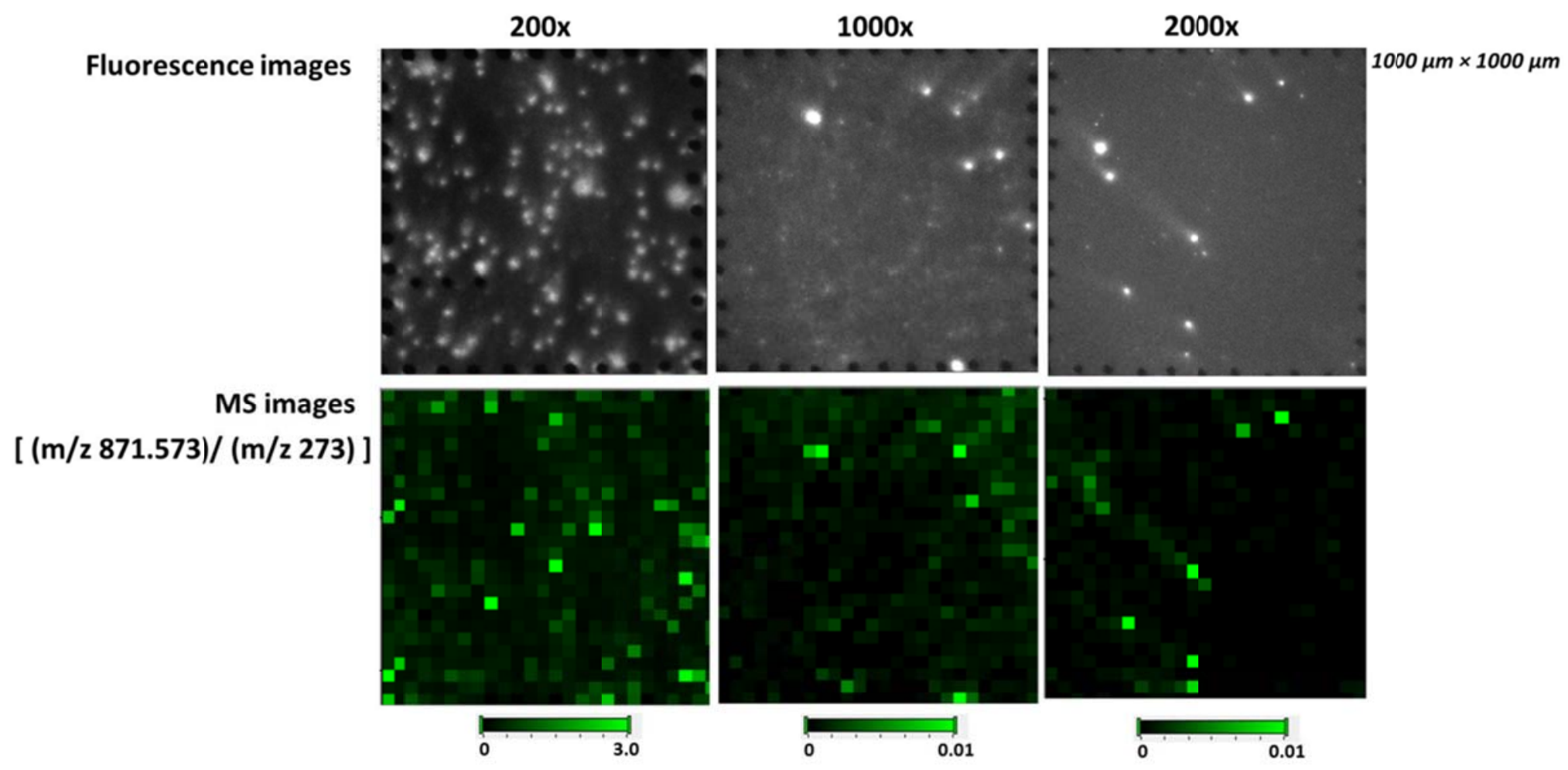

Figure 4. 
[ Signal Intensity/ (m/z 273) ]

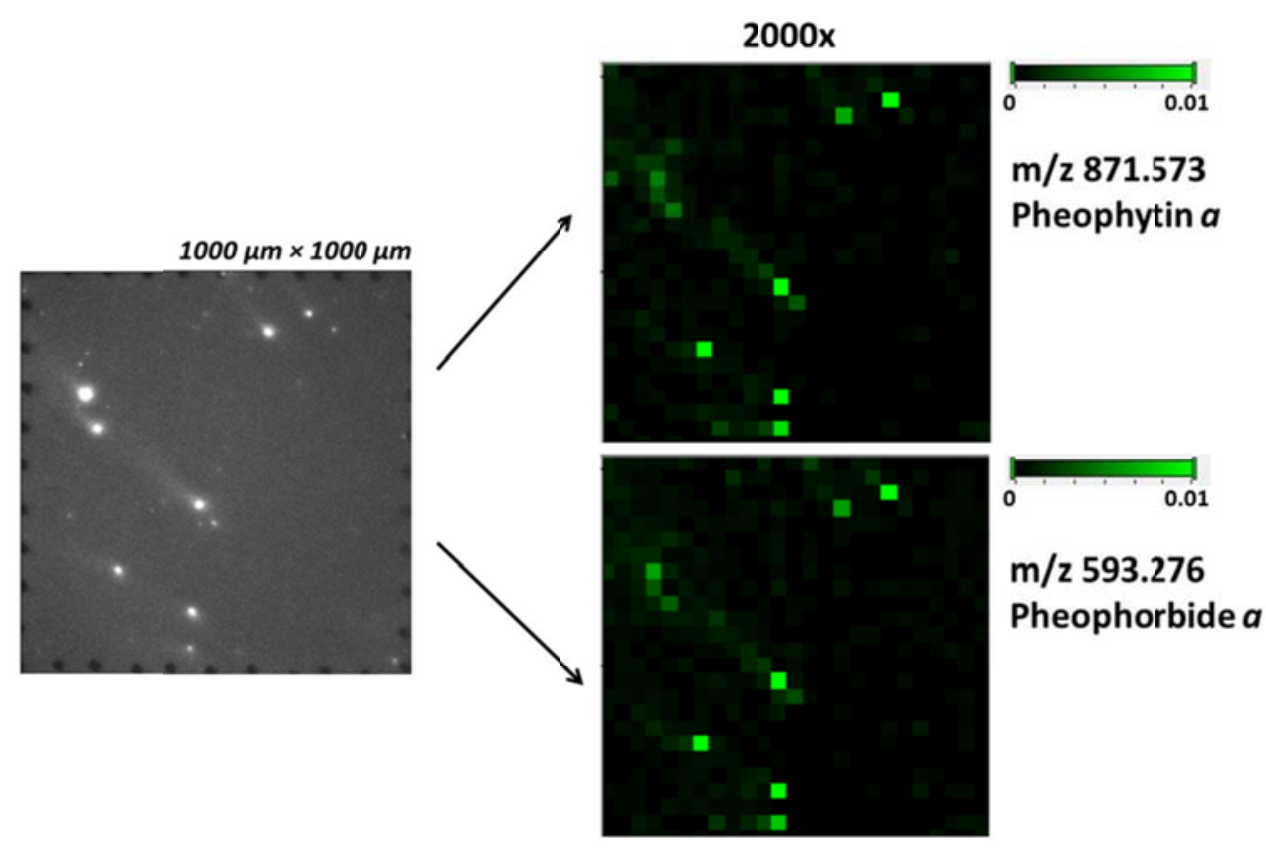

Figure 5. 


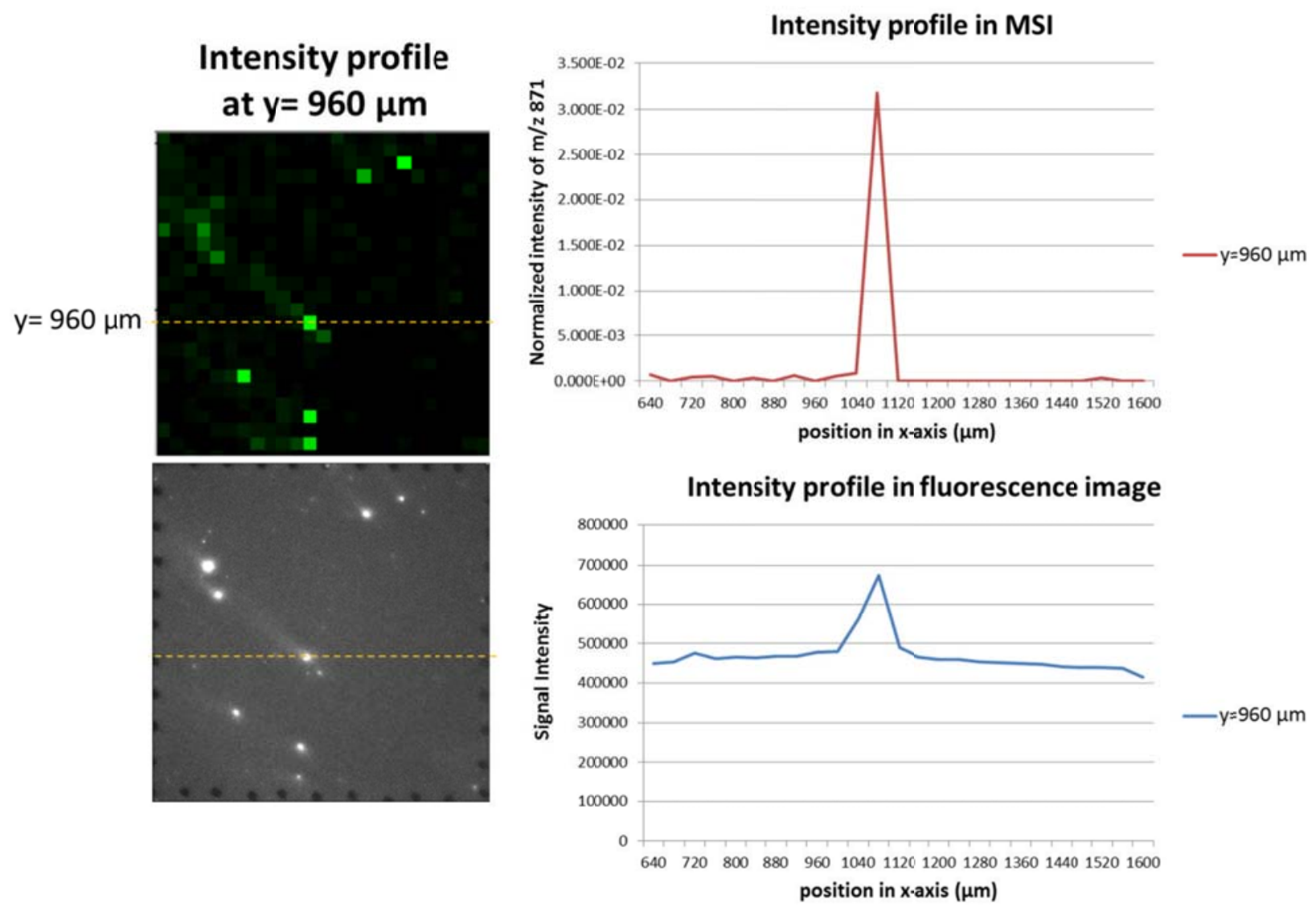

Figure 6. 

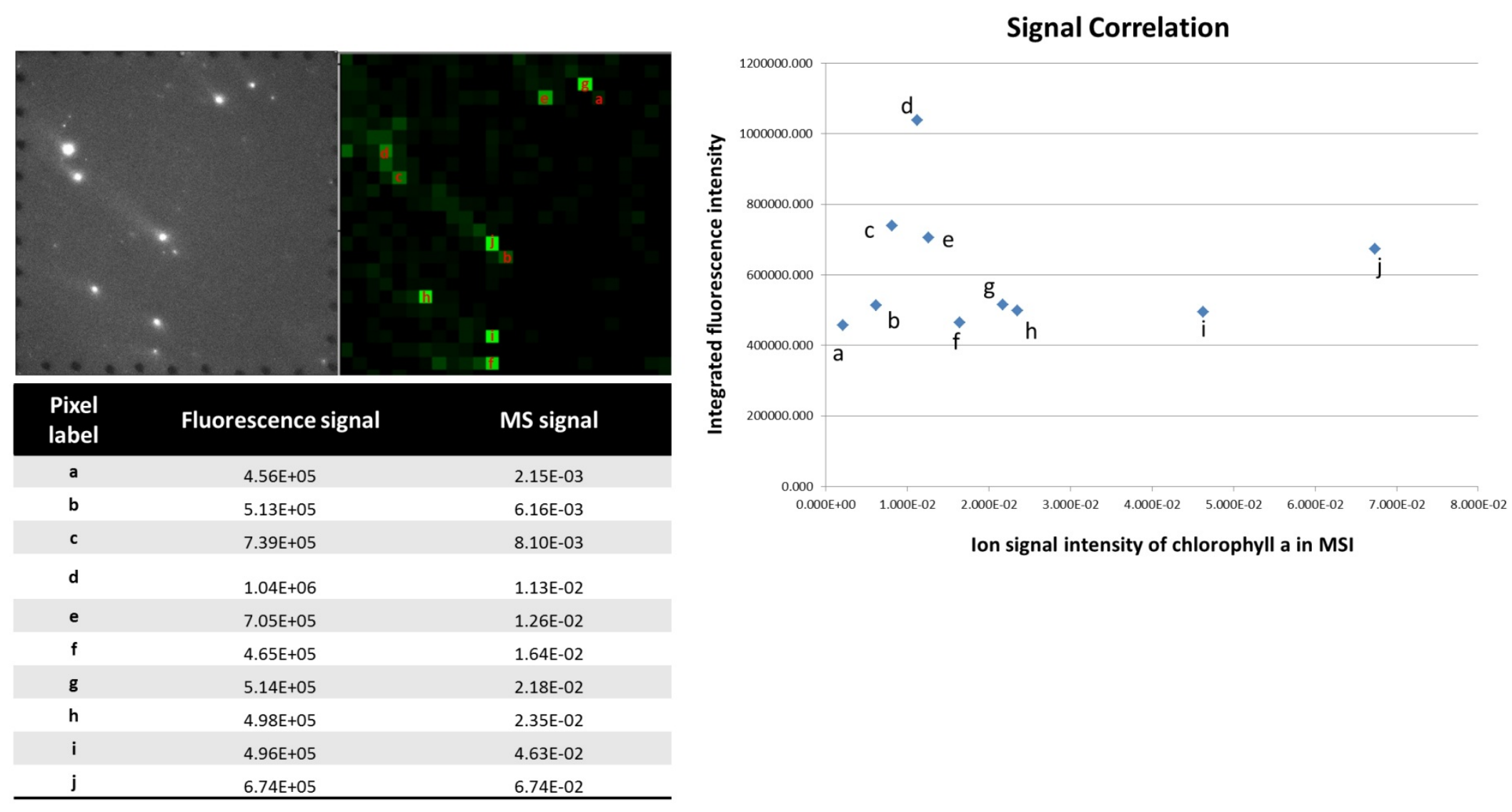

Figure 7. 


\section{CHAPTER 6. GENERAL CONCLUSIONS}

In this dissertation, high-spatial and high-mass resolution laser desorption ionization (LDI) mass spectrometric (MS) imaging technique was developed and applied to a study of small metabolites and endogenous molecules in the biological system, plant. The MS images of high quality were obtained due to the high spatial resolution of $\sim 12 \mu \mathrm{m}$. This cellular level spatial resolution was achieved by the reduction of laser spot size through the optical fiber with small core diameter $(25 \mu \mathrm{m})$ and the improved homogeneity of matrix application using oscillating capillary nebulizer. MS imaging of high-mass resolution was also achieved by utilizing the LTQOrbitrap mass spectrometer. High-mass resolution enabled the direct identification of the detected molecules on the biological tissue and differentiation of isobaric ions without the need of performing the tandem mass spectrometry (MS/MS).

The MS images of surface metabolites, epicuticular lipids, were obtained at high spatial resolution of $\sim 12 \mu \mathrm{m}$ on a whole Arabidopsis flower and the spatial distribution of these molecules was successfully unveiled even on the minute sub-organs of the flower such as stamen and carpel. The periodic fluctuation of the ion signal shown in its profiles along a series of single pixels on a stamen matches the size of the longitudinal epidermal cell and this supports our single cell level resolution. MS imaging of high mass resolution enabled it to directly identify the surface metabolites detected on the secondary roots of Arabidopsis thaliana through the chemical composition analysis and MS imaging of high spatial resolution also allowed revelation 
of a high degree of localization of those metabolites. This was the first MS imaging on the root for molecular species.

MS imaging at high-spatial and high-mass resolution was also applied to study the molecular function of CER 1 gene on each individual organ level. The change in spatial distribution and abundance level of targeted metabolites (i.e., waxes and flavonoids) were monitored on various organs including flowers, leaves, stems, and roots between the cer 1 mutant and the wild-type by MS imaging of high spatial resolution. The abundance changes of isobaric metabolites, i.e. C29 alkane vs. C28 aldehyde associated with the genetic mutation were tracked on both genotypes by acquiring the separate MS images of those molecules from MS imaging of high mass resolution. This was not previously achievable with low mass resolution mass spectrometry. The decrease in abundances of alkanes and ketones and hyper accumulation of aldehydes and C30 fatty acid were observed in several Arabidopsis organs of cer 1 mutant and this supported previous hypothesis of molecular function of $C E R 1$ gene as aldehyde decarbonylase.

The internal cell metabolites exposed to the surface after removal of surface cuticle layers by vibratome sectioning of the Arabidopsis leaf tissues and the subsequent enzymatic digestion of primary cell walls, middle lamella were also successfully identified to linolenic acid (C18:3 FA) and linoleic acid (C18:2 FA) by LDI-MS of high mass resolution. The spatial distribution of these metabolites in the internal cell layers were revealed by MS imaging.

LDI-MS imaging was also utilized to develop an assay for relative quantification of analytes at the single subcellular/organelle level. Whereas the feasibility of single-subcellular level quantification of analytes was confirmed at low density of dispersed chloroplasts based on the exact matching of the number of chloroplasts and their localization seen in the MS images with 
those in the fluorescence images, the significant obstacles were also seen related to the stability of chloroplasts. More investigation is necessary to resolve the problem associated with this, and hence make this assay for relative quantification more complete.

In conclusions, the significance of the two factors- spatial resolution and mass resolutionaffecting the quality of MS imaging data was clearly demonstrated with respect to the degrees of information one can extract from MS imaging data. In the near future, the achievement of smaller laser spot size down to a micron level will make it possible to image the fine localization of analytes even at the subcellular level. The great efforts should be also made to optimize the homogeneity of the laser beam and laser irradiance because these factors are known to make a strong influence on the signal level. In association with the further reduction of laser spot size, adoption of new types of matrix such as ionic liquid matrixes is also being pursued in our lab to enhance the sensitivity of analytes detection within a small region of the biological sample fired by laser. 


\section{APPENDIX 1. SUPPORTING FIGURES FOR CHAPTER 2}

This supporting information is published online at http:// pubs.acs.org ${ }^{*}$

Ji Hyun Jun, Zhihong Song, Zhenjiu Liu, Basil J. Nikolau, Edward S. Yeung, and Young Jin Lee

\section{Figure Captions}

Figure S-1. Photos of laser burn marks on $\alpha$-cyano-4-hydroxycinnamic acid matrix obtained with optical fibers with (a) $25 \mu \mathrm{m}$ and (b) $10 \mu \mathrm{m}$ inner core diameter in vMALDI LTQ mass spectrometer. Matrix was prepared either in 50:50 acetonitrile:water (a) or acetone (b). The raster sizes were $50 \mu \mathrm{m}$ and $30 \mu \mathrm{m}$ for photos (a) and (b), respectively.

Figure S-2. Single pixel ion abundance profiles for C29 alkane, C29 ketone, and C26 fatty acid as silver ion adducts $(\mathrm{m} / \mathrm{z} 515,529$, and 503 , respectively) normalized to silver dimer, $\left[{ }^{107} \mathrm{Ag}+{ }^{109} \mathrm{Ag}\right]^{+}(\mathrm{m} / \mathrm{z} 216)$, as shown in Figure 5B, but at $\mathrm{x}=2112 \mu \mathrm{m}$ (Top) and $\mathrm{x}=2124 \mu \mathrm{m}$ (bottom).

*http://pubs.acs.org/doi/suppl/10.1021/ac902990p Copyright (C) 2010 American Chemical Society 
a. $25 \mu \mathrm{m}$ optical fiber
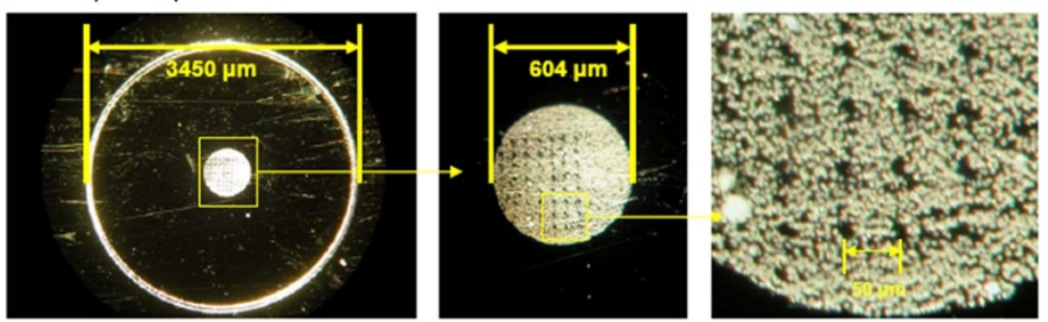

b. $10 \mu \mathrm{m}$ optical fiber

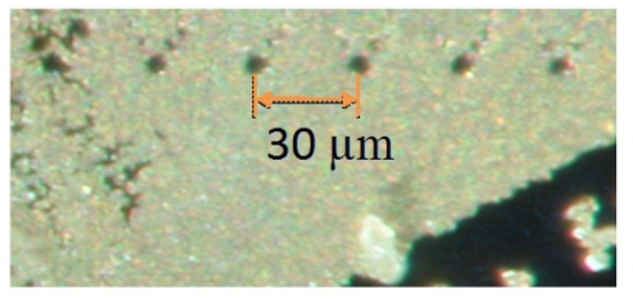

Figure S-1. 

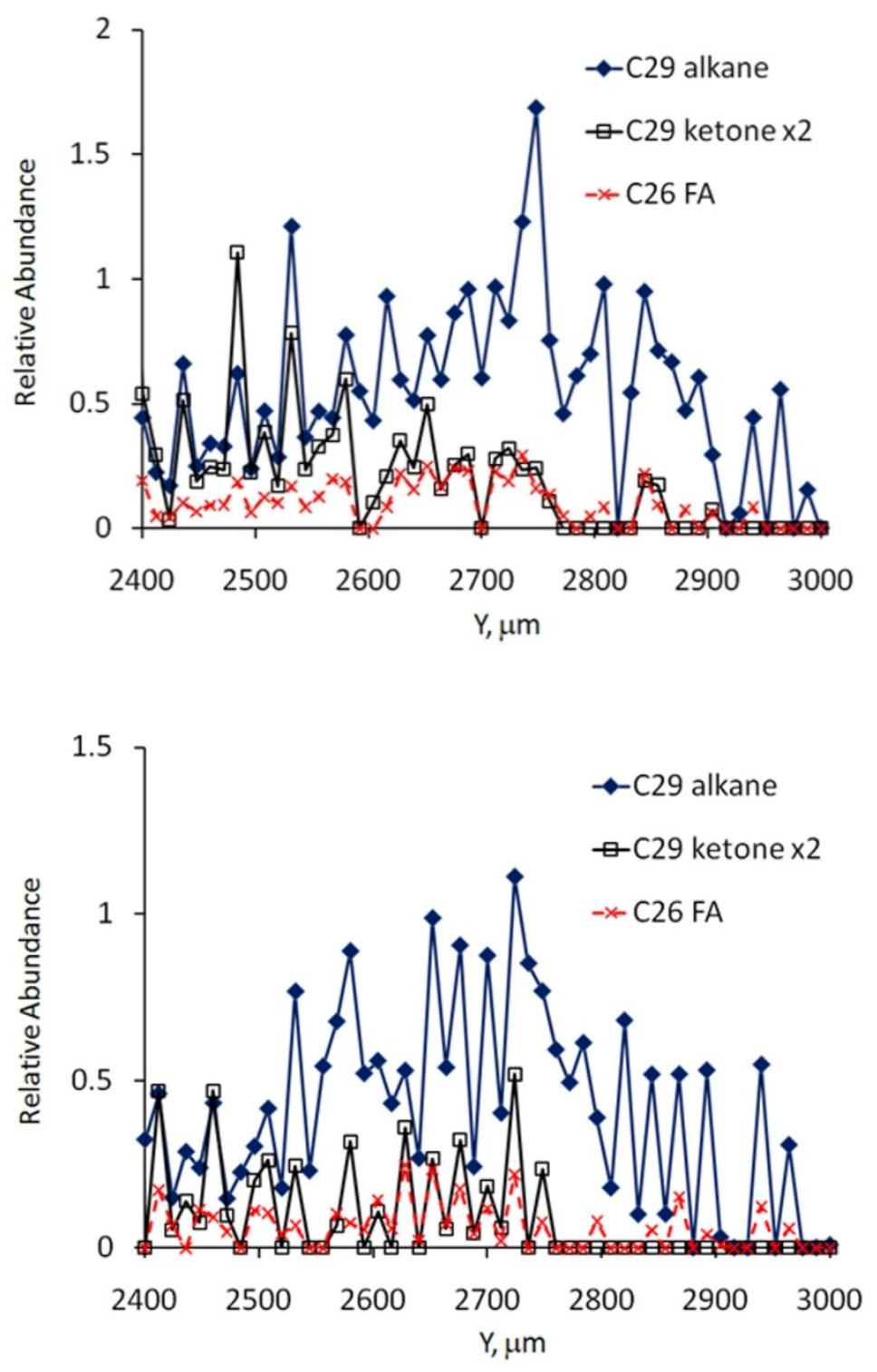

Figure S-2. 


\section{APPENDIX 2. SUPPORTING FIGURES FOR CHAPTER 3}

Ji Hyun Jun, Zhenjiu Liu, Zhihong Song, Basil J. Nikolau, Edward S. Yeung, and Young Jin Lee

\section{Figure Captions}

Figure S-1. MS imaging of fatty acids (C26-C30) and flavonoids on the Arabidopsis flowers with colloidal graphite as a matrix. The flowers were 43 days old when collected. The samples were scanned with a step size of $50 \mu \mathrm{m}$. The abudance level of metabolites on the mutant sample below the dotted line is artifact caused by damage during the sample preparation. The alteration of $C E R 1$ gene barely affects the spatial distributions and/or abundances of flavonol (Q; $m / z ~ 301$, I; $m / z 315$ ) and glycosylated flavonol (K-Rha; $m / z 431$ ). This observation was based on the fact that $C E R 1$ gene is related to biosynthesis of major cuticular wax compounds such as aldehydes, alkanes, ketones, alcohols, and esters not that of minor compound, flavonoid.

Figure S-2. MS images of representative epicuticular wax compounds on the Arabidopsis flowers scanned with different raster size (Left: $12 \mu \mathrm{m}$, Right:50 $\mu \mathrm{m}$ ). The flowers used were collected on the 42nd day and 44th day, respectively. The laser beam size was adjusted by utilizing the optical fibers with core diameter of $2 \mathrm{X}$ laser beam size. The MS images with $12 \mu \mathrm{m}$ spatial resolution are obviously much clearer and describe the minute features of chemical 
distribution better than those with spatial resolution of $50 \mu \mathrm{m}$. Thus, the improved spatial resolution allowed the detailed MS imaging of metabolites even on the stamen, a diminutive suborgan of flower. 
[ Signal Intensity/TIC ]

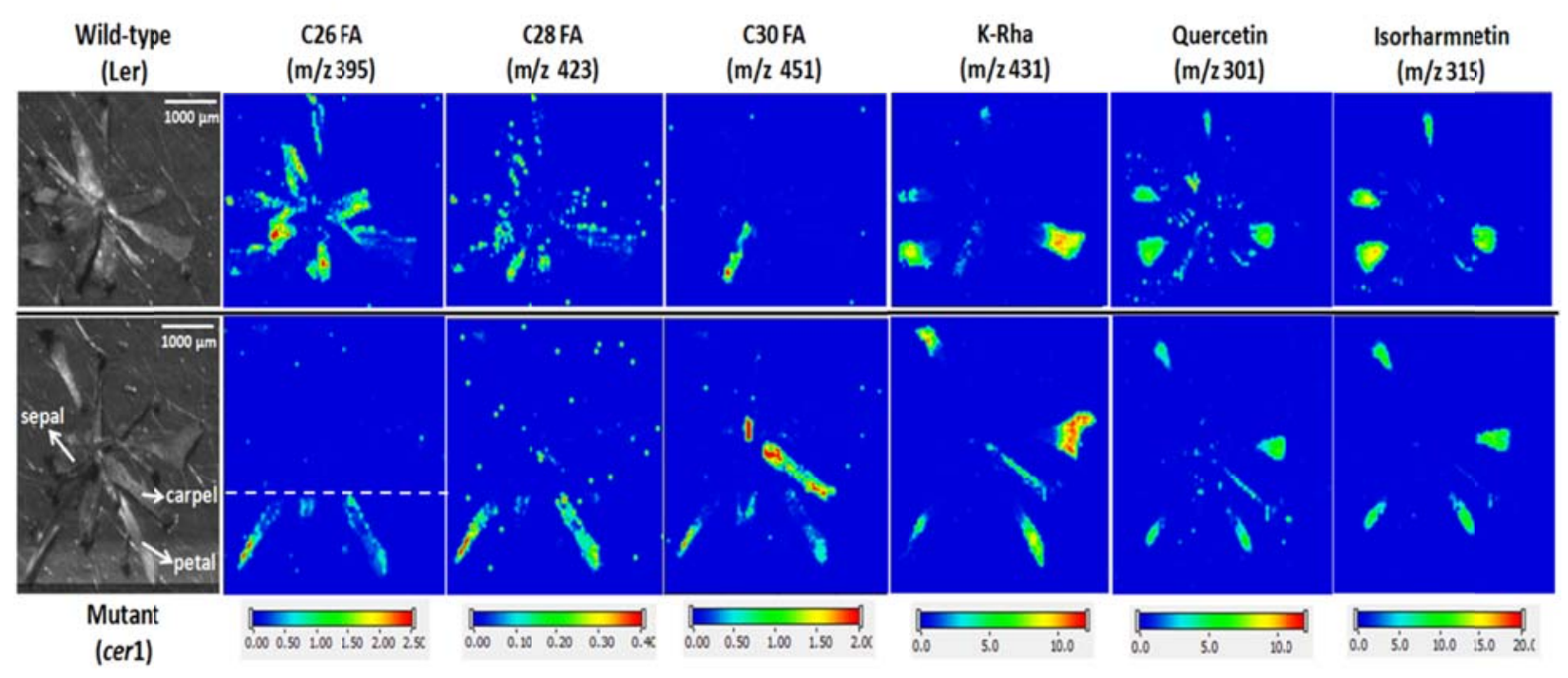

Figure S-1. 
[ Signal Intensity/ (m/z 216) ]

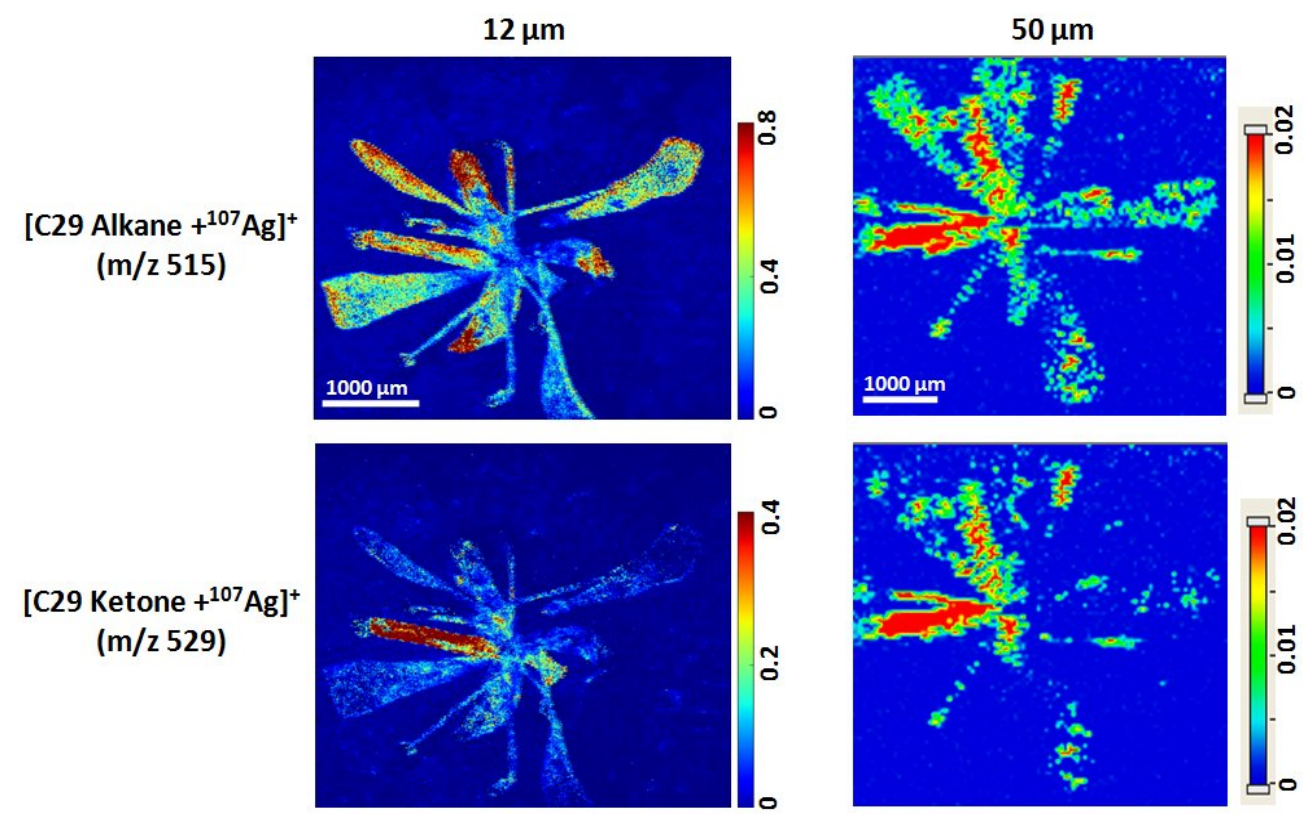

Figure S-2. 


\title{
APPENDIX 3. SUPPORTING FIGURES FOR CHAPTER 5
}

\author{
Ji Hyun Jun, Wei Sun, Basil J. Nikolau and Young Jin Lee
}

\section{Figure Captions}

Figure S-1. SEM image of sublimated DHB showing the matrix homogeneity of $\sim \leq 20 \mu \mathrm{m}$, which is homogenous enough to perform MS imaging at a spatial resolution of $40 \mu \mathrm{m}$. The homogeneous DHB layer gives the MS image of $m / z 273$ with a small variation in color over a plot range which represents the MS signal intensity. The symmetric Gaussian distribution of the number of pixels with respect to MS intensity shown in the histogram also support that the DHB applied through sublimation is highly homogeneous.

Figure S-2. Fluorescence image of isolated chloroplasts in a dilute condition spotted onto the slide without DHB coating. Much lower background signal and fluorescence free of appearance of tails from individual chloroplast are seen in this image compared to the fluorescence images shown in Figure 4. 


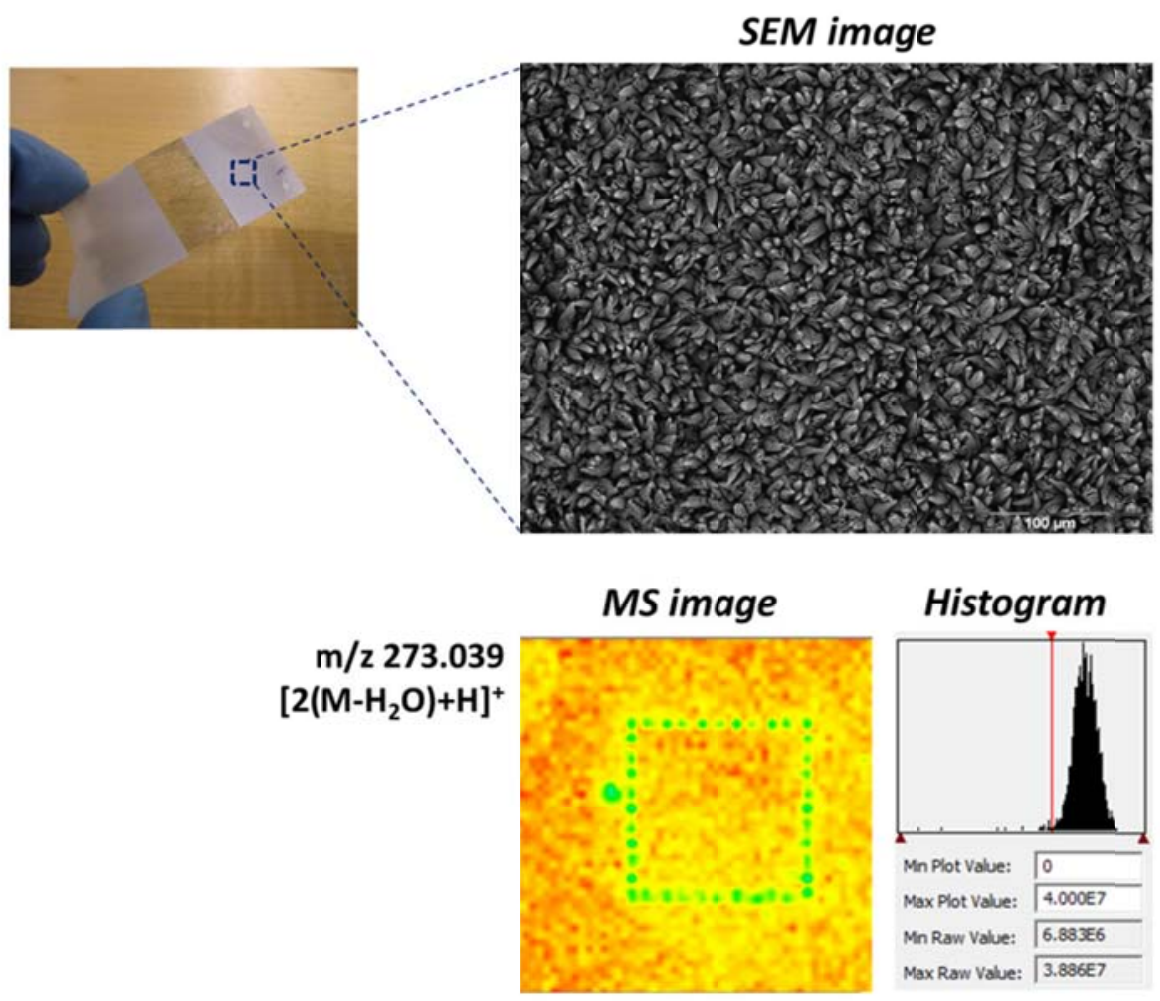

Figure S-1. 


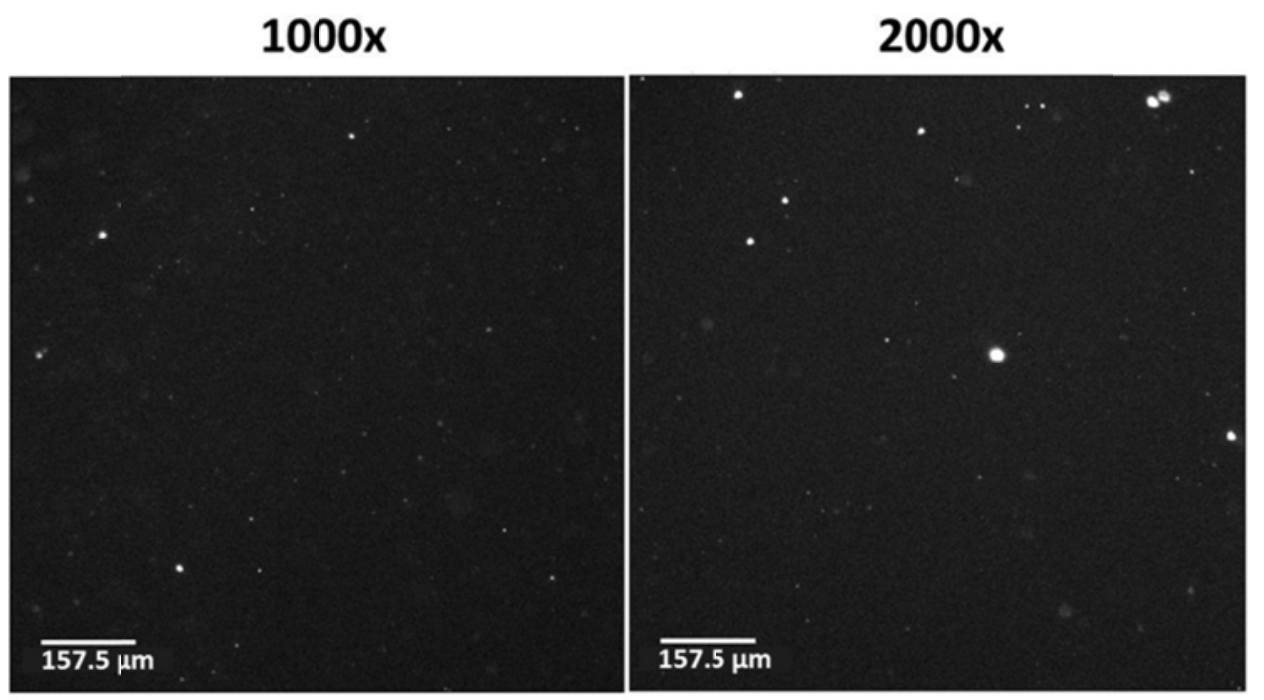

Figure S-2. 


\section{ACKNOWLEDGEMENT}

First of all, I would like to thank my advisor, Dr. Young Jin Lee for his great support and guidance on the attitude I must have as a scientist. I show my respect to his passion and efforts on the research and always learned a lot from it. Also, I want to give thanks to Dr. Hans U. Stauffer, my former major professor, for his kind advices, supports, and guidance on my path as a graduate student. I probably could not continue my graduate studies here at Iowa State without such a great help from these two important persons in my life.

I would also like to express my gratitude to all of my POS committee members, Dr. Ning Fang, Dr. Mark S. Gordon, Dr. Robert S. Houk, Dr. Theresa Windus for their encouragement and valuable advices on my research. Although he is not one of my POS committee members, it was my honor to have a chance to meet with Dr. Edwards S. Yeung even for a short period of time and to talk about my research together.

I could meet lots of good people from both the former and current research group and all of them really helped me have a nice time in the lab and enjoy working with them by showing their great supports and friendship: To Dr. Benjamin D. Prince, Beth M. Prince, Dr. Alex J. Blom, and Dr. Mikhail N. Slipchenko- I cannot forget the warm memories that we shared together in Stauffer lab and your great care on me at my first year. To Dr. David C. Perdian, Dr. Zhenjiu Liu, Dr. Soojin Park, Dr. Rachael Leverence, Erica A. Smith, Andy Korte, Adam Klein, Paul Cole, Gargey Yagnik in Lee group - I appreciate your cooperation and sincere help. I should also say that I was happy to work with great collaborators, Dr. Zhihong Song and Dr. Wei Sun. I also 
enjoyed working with Dr. Harry T. Horner, Randall L. Den Adel, and Tracy M. Pepper in the Microscopy and NanoImaging Factility and I am very grateful for their great help and advices.

Lastly, I want to express my deep gratitude especially to my best friend, Sinele Tsabedze in Pohl group. You have been always there, right beside me not only in the happy moments but also in the difficult times from the beginning of my life here in Ames until now. You will be always with me in Ames in my memory even after our graduation. I cannot thank my parents and sister enough for their unconditional love, great supports that they showed me all the time. I do not think I could complete my study without their love. I would also love to show my special thanks to my closest friends for their great supports and true friendship. 